\title{
WestVirginiaUniversity
}

THE RESEARCH REPOSITORY @ WVU

Graduate Theses, Dissertations, and Problem Reports

2005

\section{Applications of the covering property axiom}

Andres Millan Millan

West Virginia University

Follow this and additional works at: https://researchrepository.wvu.edu/etd

\section{Recommended Citation}

Millan Millan, Andres, "Applications of the covering property axiom" (2005). Graduate Theses,

Dissertations, and Problem Reports. 2249.

https://researchrepository.wvu.edu/etd/2249

This Dissertation is protected by copyright and/or related rights. It has been brought to you by the The Research Repository @ WVU with permission from the rights-holder(s). You are free to use this Dissertation in any way that is permitted by the copyright and related rights legislation that applies to your use. For other uses you must obtain permission from the rights-holder(s) directly, unless additional rights are indicated by a Creative Commons license in the record and/ or on the work itself. This Dissertation has been accepted for inclusion in WVU Graduate Theses, Dissertations, and Problem Reports collection by an authorized administrator of The Research Repository @ WVU.

For more information, please contact researchrepository@mail.wvu.edu. 


\title{
Applications of the Covering Property Axiom
}

\author{
Andrés Millán Millán \\ Dissertation submitted to the \\ Eberly College of Arts and Sciences \\ at West Virginia University \\ in partial fulfillment of the requirements \\ for the degree of \\ Doctor of Philosophy \\ in \\ Mathematics
}

Krzysztof Ciesielski Ph.D., Chair

John Atkins Ph.D.

Cun Quan Zhang Ph.D.

Sam Nadler Ph.D.

Jerzy Wojciechowski Ph.D.

Department of Mathematics

Morgantown, West Virginia

2005

Keywords: Covering Property Axiom CPA, perfect cube, prism, $Q-$ point, crowded ultrafilter, strong $\gamma$-set, separately nowhere-constant function.

Copyright 2005 Andrés Millán Millán 


\section{ABSTRACT \\ Applications of the Covering Property Axiom}

\section{Andrés Millán Millán}

The purpose of this work is two-fold. First, we present some consequences of the Covering Property Axiom CPA of Ciesielski and Pawlikowski which captures the combinatorial core of the Sacks' model of the set theory. Second, we discuss the assumptions in the formulation of different versions of CPA.

As our first application of CPA we prove that under the version $\mathrm{CPA}_{\text {cube }}^{\text {game }}$ of CPA there are uncountable strong $\gamma$-sets on $\mathbb{R}$. It is known that Martin's Axiom (MA) implies the existence of a strong $\gamma$-set on $\mathbb{R}$. Our result is

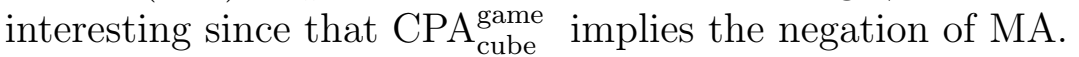

Next, we use the version $\mathrm{CPA}_{\text {prism }}^{\text {game }}$ of CPA to construct some special ultrafilters on $\mathbb{Q}$. An ultrafilter on $\mathbb{Q}$ is crowded provided it contains a filter basis consiting of perfect sets in $\mathbb{Q}$. These ultrafilters have been constructed under various hypotheses. We study the properties of being $P$-point, $Q$ point, and $\omega_{1}$-OK point and their negations, and prove under $\mathrm{CPA}_{\text {prism }}^{\text {game }}$ the existence of an $\omega_{1}$-generated crowded ultrafilter satisfying each consistent combination of these properties. We also refute an earlier claim by Ciesielski and Pawlikowski by proving under $\mathrm{CPA}_{\text {prism }}^{\text {game }}$ that there are $2^{\mathfrak{c}}$-many crowded c-generated $Q$-points.

We also study various notions of density, central to the foundation of CPA and defined in the set of all perfect subsets of a Polish space $\mathcal{X}$. These notions involve the concepts of perfect cube and iterated perfect set on $\mathfrak{C}^{\alpha}$. If $\mathcal{X}$ is a Polish space, we say that $\mathcal{F} \subseteq \operatorname{Perf}(\mathcal{X})$ is $\alpha$-cube $(\alpha$-prism) dense provided for every continuous injection $f: \mathfrak{C}^{\alpha} \rightarrow \mathcal{X}$ there exists a perfect cube (iterated perfect set) $C \subseteq \mathfrak{C}^{\alpha}$ such that $f[C] \in \mathcal{F}$.

We prove that for every $\alpha<\omega_{1}$ and every Polish space $\mathcal{X}$ there exists a family $\mathcal{F}$ such that $\mathcal{F}$ is $\beta$-prism dense for every $\beta<\alpha$ but $|\mathcal{X} \backslash \cup \mathcal{F}|=\mathfrak{c}$. Therefore, any attempt of strengthening of axiom $\mathrm{CPA}_{\text {prism }}$ by replacing prism-density with any proper subclass of these densities leads to a false statement. The proof of this theorem is based in the following result: Any separately nowhere-constant function defined on a product of Polish spaces is one-to-one on some perfect cube. 


\section{Acknowledgement}

I want to thank my thesis advisor professor Krzysztof Chris Ciesielski for his patience, guidance, and encouragement; the Mathematics Department at West Virginia University for its support and high academic level; and the members of my committee, professors John Atkins, Krzysztof Ciesielski, Sam Nadler, Jerzy Wojciechowski, and Cun-Quan Zhang. Also, I would like to thank my family and friends in Morgantown and Venezuela. Finally, I would like to say a word of love to my wife and daughter whose inconditional support allowed me to fullfill this project. 
A la memoria de mi madre 


\section{Contents}

1 Introduction and preliminaries 1

1.1 Introduction . . . . . . . . . . . . . . . . 1

1.1.1 Overview of the results . . . . . . . . . . . 1

1.1.2 Historical background . . . . . . . . . . . . 3

1.2 Notation . . . . . . . . . . . . . . . . . 5

1.3 The Covering Property Axiom . . . . . . . . . . . . 6

1.3.1 Cubes, prisms, and densities in $\operatorname{Perf}(\mathcal{X}) \ldots \ldots 6$

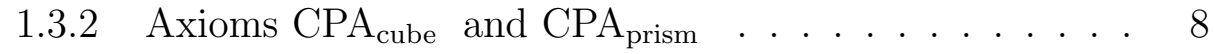

1.3.3 Games and Strategies . . . . . . . . . . . . . . . . 9 9

1.3.4 Axioms $\mathrm{CPA}_{\text {cube }}^{\text {game }}$ and $\mathrm{CPA}_{\text {prism }}^{\text {game } \ldots . . . . . . . . ~} 9$

$2 \quad \gamma$-sets and strong $\gamma$-sets. $\quad 11$

2.1 Introduction . . . . . . . . . . . . . . . . . . 11

2.2 Strong $\gamma$-sets in $\mathbb{R} \ldots \ldots \ldots$. . . . . . . . . . . . 12

3 Ultrafilters on $\mathbb{Q} \quad 17$

3.1 Preliminaries and introduction . . . . . . . . . . . . 17

3.1.1 Ideals, filters, and ultrafilters on $X \ldots \ldots \ldots 17$

3.1.2 The spaces $\beta \omega$ and $\omega^{*} \ldots \ldots \ldots \ldots$

3.1.3 Special points in $X^{*} \ldots \ldots \ldots \ldots$

3.2 Main results . . . . . . . . . . . . . . . . . . . . 23

3.3 Some important lemmas . . . . . . . . . . . . . . . . . . 24

3.4 An $\omega_{1}$-generated crowded bad point . . . . . . . . . . . . 30

3.5 A crowded $Q$-point which is not an $\omega_{1}$-OK point . . . . . . . 32

3.6 Crowded $\omega_{1}$-generated $\omega_{1}$-OK points . . . . . . . . . . . 36

3.7 Crowded $Q$-points with a large character . . . . . . . . . . . 51 
4 Separately nowhere constant functions $\quad 55$

4.1 Introduction . . . . . . . . . . . . . . . . . 55

4.2 Separately nowhere constant functions . . . . . . . . . . . 57

4.3 Cube and prism densities . . . . . . . . . . . . . . . 62

4.4 Final remarks . . . . . . . . . . . . . . . . . 67 


\section{Chapter 1}

\section{Introduction and preliminaries}

\section{$1.1 \quad$ Introduction}

\subsubsection{Overview of the results}

The purpose of this work is two-fold. First, we present some consequences of the axiom CPA of Ciesielski and Pawlikowski, in its versions $\mathrm{CPA}_{\text {cube }}^{\text {game }}$ and $\mathrm{CPA}_{\text {prism, }}^{\text {game }}$ which captures the combinatorial core of the Sacks' model. Second, we discuss the assumptions in the formulation of different versions of CPA. As our first application of CPA we prove, in Chapter 2, that under $\mathrm{CPA}_{\text {cube }}^{\text {game }}$ there are uncountable strong $\gamma$-sets on $\mathbb{R}$. Strong $\gamma$-sets belong to the realm of the small subsets of $\mathbb{R}$. To be precise, recall that a family $\mathcal{U}$ of open subsets of $\mathbb{R}$ is a cover of $Z \subseteq[\mathbb{R}]^{<\omega}$, the set of finite subsets of $\mathbb{R}$, provided for every $A \in Z$ there is a $U \in \mathcal{U}$ with $A \subseteq U$; an $S \subseteq \mathbb{R}$ is a strong $\gamma$-set provided there exists an increasing sequence $\left\langle k_{n}<\omega: n<\omega\right\rangle$ such that for every sequence $\left\langle J_{n}: n<\omega\right\rangle$, where each $J_{n}$ is an cover of $[\mathbb{R}]^{k_{n}}$, there exists a $\left\langle D_{n} \in J_{n}: n<\omega\right\rangle$ such that $S \subseteq \bigcup_{n<\omega} \bigcap_{m>n} D_{m}$. In reference [18] F. Galvin and A. Miller showed that MA implies the existence of a strong $\gamma$-set on $\mathbb{R}$. Our result is interesting since that $\mathrm{CPA}_{\text {cube }}^{\text {game }}$ implies the total failure of Martin's Axiom, and in particular, $\neg$ MA.

As our second application we use $\mathrm{CPA}_{\text {prism }}^{\text {game }}$, in Chapter 3, to construct the examples of special ultrafilters on $\omega$ and $\mathbb{Q}$. Recall that a filter on a non-empty set $X$ is a non-trivial family $\mathcal{U} \subseteq \mathcal{P}(X)$ that is closed under finite intersections and supersets and that an ultrafilter is a maximal filter. If in addition $\mathcal{U}$ does not contain finite sets, then $\mathcal{U}$ is called non-principal or free. A non-principal ultrafilter $\mathcal{U}$ on a countably infinite set $X$ is called 
a $P$-point if for every partition $\mathcal{P}$ of $X$ into pieces not in $\mathcal{U}$ there exists a $U \in \mathcal{U}$ that contains at most one element of every piece; if in the definition of $P$-point we restrict ourselves to the partitions into finite pieces, we obtain the definition of a $Q$-point. The existence of these ultrafilters is consistent with and independent of ZFC. We will also consider a class of weak $P$-points called $\omega_{1}-O K$ points which actually exist in ZFC (see [27]).

An ultrafilter on $\mathbb{Q}$ is crowded provided it contains a filter basis consiting of perfect sets in $\mathbb{Q}$, where the topolgy on $\mathbb{Q}$ is the relativization of the usual topology on $\mathbb{R}$. Although it is an open problem to decide if the existence of such ultrafilters follows from ZFC, they have been constructed under various hypothesis like MA [14], $\mathfrak{b}=\mathfrak{c}[13]$ or $\mathrm{CPA}_{\text {prism }}^{\text {game }}[9]$.

For $\omega_{1}$-generated ultrafilters ${ }^{1}$, we consider the properties of being $P$-point, $Q$-point, or $\omega_{1}$-OK point and their negations, and prove for every combination of these, the existence under $\mathrm{CPA}_{\text {prism }}^{\text {game }}$ of an ultrafilter with precisely that combination of properties, as long they do not contradict with each other. Moreover, we prove that, when considered on $\mathbb{Q}$, such ultrafilters can be crowded as well.

In one of these constructions we refute a claim of Ciesielski and Pawlikowski from [9] by proving that under $\mathrm{CPA}_{\text {prism }}^{\text {game }}$ there are $2^{\mathfrak{c}}$-many crowded c-generated $Q$-points.

In Chapter 4 we consider various notions of density defined in the family of all perfect subsets of a Polish space $\mathcal{X}$. If $0<\alpha<\omega_{1}$, a perfect cube in $\mathfrak{C}^{\alpha}$ is a set $C$ of the form $\prod_{\xi<\alpha} C_{\xi}$, where each $C_{\xi}$ is homeomorphic to the Cantor set $\mathfrak{C}$. This notion can be generalized to that of iterated perfect set in $\mathfrak{C}^{\alpha}$. Then, if $\mathcal{X}$ is a Polish space, we say that $\mathcal{F} \subseteq \operatorname{Perf}(\mathcal{X})$ is $\alpha$-cube $\left(\alpha\right.$-prism) dense provided for every continuous injection $f: \mathfrak{C}^{\alpha} \rightarrow \mathcal{X}$ there exists a perfect cube (iterated perfect set) $C \subseteq \mathfrak{C}^{\alpha}$ such that $f[C] \in \mathcal{F}$.

We prove that for every $\alpha<\omega_{1}$ and every Polish space $\mathcal{X}$ there exists a family $\mathcal{F}$ such that $\mathcal{F}$ is $\beta$-prism dense for every $\beta<\alpha$ but $|\mathcal{X} \backslash \bigcup \mathcal{F}|=\mathfrak{c}$. This shows that any attempt of strenghtening of axiom $\mathrm{CPA}_{\text {prism }}$ by replacing the prism density with any proper subclass of these densities leads to a stamement which is false in ZFC.

The main tool to prove this theorem is the following result: Any separately nowhere-constant function defined on a product of Polish spaces is one-to-one on some perfect cube. Where a function defined on a product of Polish spaces is separately nowhere-constant provided it is not constant on any section.

\footnotetext{
${ }^{1}$ These are the ultrafilters with a filter basis of cardinality $\omega_{1}$
} 


\subsubsection{Historical background}

Set Theory began in the late XIX ${ }^{\text {th }}$ century with the work of Georg Cantor about trigonometric series. At some point during his research Cantor had to consider infinite sets of real numbers. His studies led him to state the Continuum Hipothesis (CH). In 1904, Ernst Zermelo stated the Axiom of Choice (AC) and used it to prove that every set can be well-ordered [47]. Both statements were proved consistent relative to the other axioms of Set Theory $(\mathrm{ZF})^{2}$ by K. Gödel in [20], [21], and [22]. In 1960's, P.J. Cohen proved the independence of $\mathrm{CH}$ and $\mathrm{AC}$ by showing that their negations are also consistent with ZF [6]. Gödel's extremely important work was based on the detailed study of $L$, the class of constructible sets, which he proved to be a transitive model for the theory ZFC $+\mathrm{GCH}^{3}$. On the other hand, Cohen's proof made use of the method of forcing, a very powerful technique created by him that have provided a plethora of consistency results.

Roughly speaking, forcing arguments start with a ground model $M$ of ZFC, like $L$, and a forcing notion which is a partially ordered set $\langle\mathbb{P}, \leq\rangle$ that will force the existence of certain generic object $G$ in a model extension $M[G]$ of $M$. If $\langle\mathbb{P}, \leq\rangle$ is rich enough, then $G \notin M$.

Forcing has been used to establish the independence of many important mathematical statements like, Souslin Hypothesis, Borel's Conjecture, Kaplansky's Conjecture, and Whitehead's Problem. However, although it has been greatly refined and simplified, sometimes forcing arguments may require deep metamathematical ideas and be very technical and involved.

In 1970, D. A. Martin and R. Solovay [33] isolated a single principle which encoded the essence of a large family of forcing notions, the class of all countable chain condition (ccc) partial orders. This principle, known as Martin's Axiom (MA) was proved consistent relative to $\mathrm{ZFC}+\neg \mathrm{CH}$ by R. Solovay and S. Tennenbaum in [43]. Something similar was made for the model $L$ by R. Jensen [25] who isolated several combinatorial principles in order to capture the set-theoretical core of this model. One of these principles, $\diamond$ (diamond), is relevant to the solution of Souslin Problem.

As these results appeared, mathematicians became familiar with those principles proved to be consistent with the usual axioms of set theory and started to apply them to get the consistency and independence of many statements in classical mathematics. This is why $\mathrm{CH}, \mathrm{MA}$, and $\diamond$ and their vari-

\footnotetext{
${ }^{2}$ Zermelo-Fraenkel axioms for the Set Theory.

${ }^{3} \mathrm{ZFC}=\mathrm{ZF}+\mathrm{AC}, \mathrm{GCH}=$ Generalized Continuum Hypothesis: $\forall \kappa 2^{\kappa}=\kappa^{+}$.
} 
ants have became popular among many mathematicians working in different areas particularly, set-theoretical topologists, real analysts, and homological algebrists.

In [42] G. Sacks defined a forcing notion consisting of perfect sets of real numbers ordered by inclusion. The generic object $G$ for this forcing notion produces a real number $x_{G}$, called a Sacks real, which is not in the ground model $M$ but it has the following minimality property ${ }^{4}$ : If $A \in$ $M[G]=M\left[x_{G}\right]$ is any set of ordinals, then either $x_{G} \in M[A]^{5}$ or $A \in M$. There are essentially two different ways of adding many Sacks reals to a model of Set Theory. One way consist of adding all the Sacks reals simultaneously by using a product of many Sacks forcings. This method is called adding Sacks reals side-by-side. The other way is to add one real at a time by using an iterated forcing construction. To this thesis, the most relevant of these constructions is the one starting with a model of $\mathrm{ZFC}+\mathrm{CH}$ and adding $\omega_{2}$ Sacks reals iteratively with countable support ${ }^{6}$. The model so obtained is called the iterated Sacks model or the iterated perfect-set model and it is denoted by $\mathbb{S}$. In 1979 J. Baumgartner and R. Laver proved that in this model, every selective ultrafilter is generated by $\omega_{1}$-many sets and every selective ultrafilter in the ground model generates a selective ultrafilter in the extension. Since the equality $2^{\omega_{1}}=\omega_{2}$ may hold in $\mathbb{S}$, this result implies that in $\mathbb{S}$, there are only such a model $\omega_{2}$-many selective ultrafilters. Another interesting result about $\mathbb{S}$ is the fact proved by A. Miller in [38], that every $A \subseteq \mathbb{R}$ such that $|A|=\mathfrak{c}$ can be continuously mapped onto $[0,1]$.

Associated to $\mathbb{R}$ (the continuum) are some cardinals called the cardinal characteristics or cardinal invariants of the continuum. These cardinals are uncountable and, in general, they are defined as the minimum size of a certain families in $[\omega]^{\omega}, \omega^{\omega}$, or $\mathcal{P}(\mathbb{R})$. In presence of MA all these cardinals are equal to $\mathfrak{c}$. However, all of them are equal to $\omega_{1}$ in $\mathbb{S}$ (see [4]). This gives to $\mathbb{S}$ certain canonical character among all the models of ZFC.

In [9] K. Ciesielski and J. Pawlikowski isolated a single mathematical principle to capture the combinatorial core of $\mathbb{S}$. They call it the Covering Property Axiom and denoted it CPA. However, they used mainly a weakened, but still prominent, version of it denoted $\mathrm{CPA}_{\text {prism }}^{\text {game }}$. They claimed that even this weaker version of CPA captures the combinatorial core of $\mathbb{S}$, at least

\footnotetext{
${ }^{4}$ See $[24$, Theorem 64].

${ }^{5} M[A]$ is the least model of ZFC containing $M$ and $A$.

${ }^{6}$ For the terminology of forcing and iterated forcing see [30] or [24].
} 
from the point of view of the cardinal invariants of the continuum. This claim is supported for two facts. First, Ciesielski and Pawlikowski proved that essentially every relevant fact that holds in $\mathbb{S}$ follows from $\mathrm{CPA}_{\text {prism }}^{\text {game }}$ (see [10]). Second, it is a result of J. Zapletal [46] that for every tame cardinal invariant $\kappa$, if the inequality $\kappa<\mathfrak{c}$ holds in any forcing extension of a model of ZFC, then $\kappa<\mathfrak{c}$ already follows from $\mathrm{CPA}_{\text {prism }}^{\text {game }}$. Reference [10] contains a formulation of the full CPA and a proof that it holds in $\mathbb{S}$.

\subsection{Notation}

Throughout what follows we will use standard terminology and notation as in [24]. In particular, lowercase letters $i, j, k, l, m, n, r$ will represent nonnegative integers. Lowercase greek letters will denote ordinals which will be identified with the set of its predecesors. Therefore, $2=\{0,1\}$ and $\omega$ will denote the first infinite ordinal and the set of non-negative integers $\{0,1, \ldots\}$, respectively. The first uncountable cardinal will be denoted as $\omega_{1}$ and we will use $\kappa$ to denote any other uncountable cardinal. If $X$ and $Y$ are non-empty sets the symbol $Y^{X}$ will denote the set of all functions from $X$ to $Y$. In particular, if $X=\omega$, then $Y^{\omega}$ will represent the set of all infinite sequences of elements in $Y$. This set can be topogized by taking the discrete topology on $Y$ and then the product topology. If $Y=2$ then we obtain the familiar Cantor space that will be denoted by $\mathfrak{C}$. If $Y=\omega$ then we obtain the Baire space $\omega^{\omega}$. The characteristic function of a set $A \subseteq \omega$ will be denoted as $\chi_{A}$. The symbol $|X|$ will denote the cardinality of $X$ and $\mathcal{P}(X),[X]^{<\omega},[X]^{\omega}$ and $[X]^{m}$ will represent the set of all subsets of $X$, the set of all finite subsets of $X$, the set of all subsets of $X$ of cardinality $\omega$, and the set of all subsets of $X$ of cardinality $m$, respectively. The cardinality of the set of real numbers $\mathbb{R}$ will be denoted as $\mathfrak{c}$. The set of rational numbers will be denoted as usual by $\mathbb{Q}$. Letters $\mathcal{A}, \mathcal{B}, \mathcal{I}, \mathcal{J}$, and $\mathcal{K}$ will denote, in general, the families of sets like countable collections in $[X]^{\omega}$ for a suitable $X$, basis or subbases for topologies, ideals and filters. Topological spaces will be denoted as $\mathcal{X}$, $\mathcal{Y}$, and $\mathcal{Z}$. If $\mathcal{X}$ is a topological space, then $C(\mathcal{X})$ will denote the space of all real valued continuous functions on $\mathcal{X}$ with the topology of pointwise convergence. If $\mathcal{X}$ is a topological space and $A \subseteq \mathcal{X}$, we will denote by $\bar{A}$ the closure of $A$ and by $\operatorname{bd}(A)$ its boundary. A Polish space is a separable and

\footnotetext{
${ }^{7}$ See reference [46] for the definition of tame cardinal invariant.
} 
completely metrizable space. The spaces $\mathbb{R}, \mathfrak{C}, \omega^{\omega}$ are examples of Polish spaces. Our references for the theory of Polish spaces are [31] and [26].

\subsection{The Covering Property Axiom}

In this section we define the main notions needed for the formulation of the main versions of the Covering Property Axiom and we state some results of crucial importance for the subsequent chapters.

\subsubsection{Cubes, prisms, and densities in $\operatorname{Perf}(\mathcal{X})$}

The framework of CPA rests on the concept of a cube and prism. For every Polish space $\mathcal{X}$ consider the set

$$
\operatorname{Perf}(\mathcal{X})=\{C \subseteq \mathcal{X}: C \text { is homeomorphic to } \mathfrak{C}\}
$$

If $0<\alpha<\omega_{1}$ is an ordinal a perfect $\alpha$-cube is a set $C \subseteq \mathfrak{C}^{\alpha}$ of the form $C=\prod_{\xi<\alpha} C_{\xi}$ such that $C_{\xi} \in \operatorname{Perf}(\mathfrak{C})$ for every $\xi<\alpha$. If $\alpha=\omega$ we call $C$ a perfect cube. A set $C \in \operatorname{Perf}(\mathcal{X})$ is called an $\alpha$-cube on $\mathcal{X}$ if there exists a perfect $\alpha$-cube and a continuous injection $f: \mathfrak{C}^{\alpha} \rightarrow \mathcal{X}$ such that $P=f[C]$.

If $0<\alpha<\omega_{1}$ is an ordinal let $\Phi_{\text {prism }}(\alpha)$ be the set of all continuous injections $f: \mathfrak{C}^{\alpha} \rightarrow \mathfrak{C}^{\alpha}$ with the property that

$$
f(x)\left\lceil\xi=f(y)\left\lceil\xi \Longleftrightarrow x \left\lceil\xi=y\left\lceil\xi \quad \text { for all } \xi<\alpha \text { and } x, y \in \mathfrak{C}^{\alpha} .\right.\right.\right.\right.
$$

Then, we define $\mathbb{P}_{\alpha}=\left\{\operatorname{range}(f): f \in \Phi_{\text {prism }}(\alpha)\right\}$ and $\mathbb{P}_{\omega_{1}}=\bigcup_{0<\alpha<\omega_{1}} \mathbb{P}_{\alpha}$. The elements of $\mathbb{P}_{\omega_{1}}$ are called the iterated perfect sets. The simplest elements of $\mathbb{P}_{\alpha}$ are of the form $C=\prod_{\xi<\alpha} C_{\xi}$, where $C_{\xi} \in \operatorname{Perf}(\mathfrak{C})$ for every $\xi<\alpha$. We refer to them as perfect cubes.

Observe that the set $\Phi_{\text {prism }}(\alpha)$ can be also described as the family of all continuous injections $f: \mathfrak{C}^{\alpha} \rightarrow \mathfrak{C}^{\alpha}$ such that for every $\beta<\alpha$

$$
f \uparrow \beta \stackrel{\text { def }}{=}\{\langle x \uparrow \beta, y \uparrow \beta\rangle:\langle x, y\rangle \in f\}
$$

is a one-to-one function from $\mathfrak{C}^{\beta}$ into $\mathfrak{C}^{\beta}$. For example, if $\alpha=3=\{0,1,2\}$ then $f \in \Phi_{\text {prism }}(\alpha)$ provided there exist continuous functions $f_{0}: \mathfrak{C} \rightarrow \mathfrak{C}$, $f_{1}: \mathfrak{C}^{2} \rightarrow \mathfrak{C}$, and $f_{2}: \mathfrak{C}^{3} \rightarrow \mathfrak{C}$ such that

$$
f\left(x_{0}, x_{1}, x_{2}\right)=\left\langle f_{0}\left(x_{0}\right), f_{1}\left(x_{0}, x_{1}\right), f_{2}\left(x_{0}, x_{1}, x_{2}\right)\right\rangle
$$


for all $x_{0}, x_{1}, x_{2} \in \mathfrak{C}$ and maps $f_{0},\left\langle f_{0}, f_{1}\right\rangle$, and $f$ are one-to-one. Note that $\Phi_{\text {prism }}(\alpha)$ is closed under compositions and that for every $0<\beta<\alpha$ if $f \in \Phi_{\text {prism }}(\alpha)$, then $f \uparrow \beta \in \Phi_{\text {prism }}(\beta)$.

The following properties can be easily deduced from these definitions. (For (D) see $[10,(3.13)]$.$) Here \pi_{\beta}$ is the projection from $\mathfrak{C}^{\alpha}$, for some $\alpha \geq \beta$, onto the first $\beta$ coordinates, that is, $\pi_{\beta}(x)=x \uparrow \beta$.

(A) Every perfect cube in $\mathfrak{C}^{\alpha}$ belongs to $\mathbb{P}_{\alpha}$.

(B) If $P \in \mathbb{P}_{\alpha+1}$ and $x \in \pi_{\alpha}[P]$, then $|(\{x\} \times \mathfrak{C}) \cap P|=\mathfrak{c}$.

(C) If $0<\beta<\alpha$ and $P \in \mathbb{P}_{\alpha}$, then $\pi_{\beta}[P] \in \mathbb{P}_{\beta}$.

(D) If $0<\beta<\alpha$, then $Q=\left\{x \in P: \pi_{\beta}(x) \in R\right\} \in \mathbb{P}_{\alpha}$ for every $P \in \mathbb{P}_{\alpha}$ and $R \in \mathbb{P}_{\beta}$ with $R \subset \pi_{\beta}[P]$.

If $\mathcal{X}$ is a Polish space, then a prism in $\mathcal{X}$ is a pair $\langle f, P\rangle$ where $f: E \rightarrow \mathcal{X}$ is injective and continuous, $E \in \mathbb{P}_{\omega_{1}}$, and $P=f[E]$. Function $f$ can be considered as a coordinate system imposed on $P$. We will usually abuse this terminology and refer to $P$ itself as a prism. In this case function $f$, given only implicitly, will be referred to as a witness function for $P$. If the domain of the witness function of a prism $P$ happens to be a perfect cube, we will sometimes refer also to $P$ as a cube in $\mathcal{X}$.

If $\langle f, P\rangle$ is a prism, then we say that $Q$ is its subprism provided there exists an iterated perfect set $E \subseteq \operatorname{dom}(f)$ such that $Q=f[E]$. We will refer to $Q$ as a subcube of $P$ when $E$ is a perfect cube.

Remark 1.1 If we need to prove that a prism $P$ contains a subprism $Q$ with some "nice property," we can always assume that the witness function $f$ for $P$ is defined on the entire set $\mathfrak{C}^{\alpha}$.

Proof. Indeed, assume that we can find a desired subprism $Q$ of a prism $P$ as long as its witness function $f$ is defined on the entire set $\mathfrak{C}^{\alpha}$. Next, take an arbitrary witness function $g$ from $E \in \mathbb{P}_{\alpha}$ onto $P$ and let $h \in \Phi_{\text {prism }}(\alpha)$ be onto $E$. Then $f=g \circ h$ is a continuous injection from $\mathfrak{C}^{\alpha}$ onto $P$, so by the above assumption we can find a subprism $Q$ of $\langle f, P\rangle$ with the "nice property" we are after. To finish the argument it is enough to note that $Q$ is also a subprism of $\langle g, P\rangle$. Indeed, since $Q=f\left[E_{0}\right]$ for some $E_{0} \in \mathbb{P}_{\alpha}$, there exists an $h_{0} \in \Phi_{\text {prism }}(\alpha)$ onto $E_{0}$. But then $h \circ h_{0} \in \Phi_{\text {prism }}(\alpha)$ 
and $Q=f\left[E_{0}\right]=(g \circ h)\left[h_{0}\left[\mathfrak{C}^{\alpha}\right]\right]=g\left[h \circ h_{0}\left[\mathfrak{C}^{\alpha}\right]\right]$ is a subprism of $\langle g, P\rangle$ as $h \circ h_{0}\left[\mathfrak{C}^{\alpha}\right] \in \mathbb{P}_{\alpha}$.

Since in the game defined below we will need to consider singletons in the same position as prisms as defined above, in what follows singletons will be considered as prisms. If $P$ is a singleton in $\mathcal{X}$ then its only subprism is $P$ itself.

The following theorem is one of the principal tools for finding subprism of a prism, so also for using CPA. This result is a refinement of a theorem proved independently by H.G. Eggleston [15] and M.L. Brodskiŭ [5].

Proposition 1.2 (K. Ciesielski and J. Pawlikowski, [10, claim 1.1.5]) Let $0<\alpha<\omega_{1}$ and consider $\mathfrak{C}^{\alpha}$ with its usual topology and its usual product measure. If $G$ is a Borel subset of $\mathfrak{C}^{\alpha}$ which is either of second category or of positive measure, then $G$ contains a perfect cube $E$. In particular $E \in \mathbb{P}_{\alpha}$.

Strictly speaking, in [10, claim 1.1.5] (see also [9, claim 2.3]) the result is proved only for $\alpha=\omega$. But this easily implies the above version.

We will need also the following fusion lemma, which is an easy compilation of Lemmas 3.1.1 and 3.1.2 from [10]. The proof of the compilation is identical to that of $[10$, cor. 3.1.3].

Proposition 1.3 (K. Ciesielski and J. Pawlikowski [10]) Let $0<\alpha<\omega_{1}$ and for every $n<\omega$ let $\mathcal{D}_{n} \subseteq\left[\mathbb{P}_{\alpha}\right]^{<\omega}$ be a family of pairwise disjoint sets such that $\emptyset \in \mathcal{D}_{n}, \mathcal{D}_{n}$ is closed under refinements, and

$(\dagger)$ for every $\mathcal{E} \in \mathcal{D}_{n}$ and $E \in \mathbb{P}_{\alpha}$ which is disjoint with $\bigcup \mathcal{E}$ there exists an $E^{\prime} \in \mathbb{P}_{\alpha} \cap \mathcal{P}(E)$ such that $\left\{E^{\prime}\right\} \cup \mathcal{E} \in \mathcal{D}_{n}$.

Then for every $n<\omega$ there is a family $\mathcal{E}_{n}=\left\{E_{k}: k<2^{n}\right\} \in \mathcal{D}_{n}$ of pairwise disjoint sets such that $E=\bigcap_{n<\omega} \cup \mathcal{E}_{n} \in \mathbb{P}_{\alpha}$.

\subsubsection{Axioms $\mathrm{CPA}_{\text {cube }}$ and $\mathrm{CPA}_{\text {prism }}$}

Let $\mathcal{X}$ be a Polish space, $0<\alpha<\omega_{1}$ and let $\mathcal{E} \subseteq \operatorname{Perf}(\mathcal{X})$. We say $\mathcal{E}$ is cube-dense provided for every continuous injection $f: \mathfrak{C}^{\omega} \rightarrow \mathcal{X}$ there exists a perfect cube $C$ such that $f[C] \in \mathcal{E}$. Similarly we say that $\mathcal{E}$ is prism-dense provided for every $0<\alpha<\omega_{1}$ and every continuous injection $f: \mathfrak{C}^{\alpha} \rightarrow \mathcal{X}$ there exists an iterated perfect set $C \in \mathbb{P}_{\alpha}$ such that $f[C] \in \mathcal{E}$. These notions will be generelized and compared in Chapter 4 . 
$\mathrm{CPA}_{\text {cube }}: \mathfrak{c}=\omega_{2}$ and for any Polish space $\mathcal{X}$ and $\mathcal{E} \subseteq \operatorname{Perf}(\mathcal{X})$ cube-dense there exists an $\mathcal{E}_{0} \in[\mathcal{E}]^{\leq \omega_{1}}$ such that $\left|\mathcal{X} \backslash \bigcup \mathcal{E}_{0}\right| \leq \omega_{1}$.

$\mathrm{CPA}_{\text {prism }}: \mathfrak{c}=\omega_{2}$ and for any Polish space $\mathcal{X}$ and $\mathcal{E} \subseteq \operatorname{Perf}(\mathcal{X})$ prism-dense there exists an $\mathcal{E}_{0} \in[\mathcal{E}]^{\leq \omega_{1}}$ such that $\left|\mathcal{X} \backslash \bigcup \mathcal{E}_{0}\right| \leq \omega_{1}$.

\subsubsection{Games and Strategies}

For a Polish space $\mathcal{X}$ consider the following game $\operatorname{GAME}_{\text {prism }}(\mathcal{X})$ of length $\omega_{1}$ played by two players, Player I and Player II. At each stage $\xi<\omega_{1}$ of the game Player I can play an arbitrary prism $P_{\xi}$ in $\mathcal{X}$ (i.e., $P_{\xi}$ is either a singleton in $\mathcal{X}$ or it belongs to $\operatorname{Perf}(\mathcal{X})$ and comes with a witness function) and Player II must respond by playing a subprism $Q_{\xi}$ of $P_{\xi}$. The game $\left\langle\left\langle P_{\xi}, Q_{\xi}\right\rangle: \xi<\omega_{1}\right\rangle$ is won by Player I provided

$$
\mathcal{X}=\bigcup_{\xi<\omega_{1}} Q_{\xi}
$$

otherwise Player II wins. A strategy for Player II is any function $S$ such that $S\left(\left\langle\left\langle P_{\eta}, Q_{\eta}\right\rangle: \eta<\xi\right\rangle, P_{\xi}\right)$ is a subprism of $P_{\xi}$ for every partial game $\left\langle\left\langle P_{\eta}, Q_{\eta}\right\rangle: \eta<\xi\right\rangle$. We say that a game $\left\langle\left\langle P_{\xi}, Q_{\xi}\right\rangle: \xi<\omega_{1}\right\rangle$ is played according to a strategy $S$ for Player II provided $Q_{\xi}=S\left(\left\langle\left\langle P_{\eta}, Q_{\eta}\right\rangle: \eta<\xi\right\rangle, P_{\xi}\right)$ for every $\xi<\omega_{1}$. A strategy $S$ for Player II is a winning strategy provided Player II wins any game played according the strategy $S$. Similarly, we can define the game $\operatorname{GAME}_{\text {cube }}(\mathcal{X})$ where instead of prisms each player play a cube in $\mathcal{X}$ and strategies are defined along the same lines but replacing the word prism by cube. This leads us to the main versions of CPA.

\subsubsection{Axioms $\mathrm{CPA}_{\text {cube }}^{\text {game }}$ and $\mathrm{CPA}_{\text {prism }}^{\text {game }}$}

The following principles capture the combinatorial core of the iterated perfect set model.

$\mathrm{CPA}_{\text {prism }}^{\text {game }}: \mathfrak{c}=\omega_{2}$ and for any Polish space $\mathcal{X}$ Player II has no winning strategy in the game $\operatorname{GAME}_{\text {prism }}(\mathcal{X})$.

$\mathrm{CPA}_{\text {cube }}^{\text {game }}: \quad \mathfrak{c}=\omega_{2}$ and for any Polish space $\mathcal{X}$ Player II has no winning strategy in the game $\operatorname{GAME}_{\text {cube }}(\mathcal{X})$. 
These axioms are consequences of a slightly more general principle, similar in spirit, called CPA, see [10]. Its importance comes from the following theorem.

Proposition 1.4 (K. Ciesielski and J. Pawlikowski [10, thm. 7.2.1]) CPA holds in the iterated perfect set model. In particular, CPA is consistent with ZFC set theory.

The proof that $\mathrm{CPA}_{\text {prism }}^{\text {game }}$ holds in $\mathbb{S}$ can be also found in $[9$, thm. 5.3]. The following diagram shows the logical dependence between the different versions of CPA discussed in this work. It is not known if any of this implications is reversible.

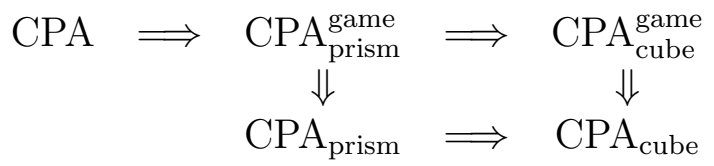




\section{Chapter 2}

\section{$\gamma$-sets and strong $\gamma$-sets.}

\section{$2.1 \quad$ Introduction}

We say that a topological space $\mathcal{X}$ is Fréchet if for every $x \in \bar{A} \subseteq \mathcal{X}$, there exists a sequence in $A$ which converges to $x^{1}$. It is natural to ask about what properties of $\mathcal{X}$ imply that the space $C(\mathcal{X})$ is Fréchet.

Definition 2.1 If $\mathcal{X}$ is a topological space and $\mathcal{J}$ is a family of open subsets of $\mathcal{X}$ then $\mathcal{J}$ is an $\omega$-cover of $\mathcal{X}$ if and only if every finite subset of $\mathcal{X}$ is contained in an element of $\mathcal{J}$.

Definition 2.2 A topological space $\mathcal{X}$ has the $\gamma$-property if and only if for every $\omega$-cover $\mathcal{J}$ of $\mathcal{X}$ there exists a sequence $\left\langle D_{n}: n<\omega\right\rangle \in \mathcal{J}^{\omega}$ such that

$$
\mathcal{X} \subseteq \bigcup_{m} \bigcap_{n>m} D_{n}
$$

In [34] and [19] is shown that $C(\mathcal{X})$ is Fréchet if and only if $\mathcal{X}$ has the $\gamma$-property.

Definition 2.3 $X \subseteq \mathbb{R}$ is a $\gamma$-set provided that $X$ has the $\gamma$-property considered as a topological space with the subsespace topology inherited from the usual topology on $\mathbb{R}$.

\footnotetext{
${ }^{1}$ See $[16]$ for more about sequential spaces.
} 
In [18] Galvin and Miller used Martin's axiom to construct several kinds of uncountable $\gamma$-sets. These have been extensively studied in the literature of singular subsets of $\mathbb{R}$. See for example [39], [2], [41].

Recall that $X \subseteq \mathbb{R}$ is a strongly measure zero set provided $X+G \neq \mathbb{R}$ for every meager subset $G$ of $\mathbb{R}$ and a $X \subseteq \mathbb{R}$ is a strongly meager set provided $X+G \neq \mathbb{R}$ for every measure zero subset $G$ of $\mathbb{R}$. This a notion that are dual of each other.

Now, although every $\gamma$-set is a strong measure zero set, T. Bartoszysńki and I. Reclaw [2] constructed, under Martin's axiom, a $\gamma$-set $T$ in $\mathbb{R}$ that is not strongly meager. The existence of such a set under axiom $\mathrm{CPA}_{\text {cube }}^{\text {game has }}$ been proved by Ciesielski and Pawlikowski in [7].

In the next section we will show that axiom $\mathrm{CPA}_{\text {cube }}^{\text {game }}$ also implies the existence of an uncountable $\gamma$-set $X \subseteq \mathbb{R}$ that is strongly meager.

\section{$2.2 \quad$ Strong $\gamma$-sets in $\mathbb{R}$}

Definition 2.4 Let $\mathcal{X}$ be a Polish space with topology $\tau$. We say that $\mathcal{U} \subseteq \tau$ is a cover of $Z \subseteq[\mathcal{X}]^{<\omega}$ provided for every $A \in Z$ there is a $U \in \mathcal{U}$ with $A \subseteq U$.

Following [18] we say that

Definition 2.5 A set $S \subseteq \mathcal{X}$ is a strong $\gamma$-set provided there is an increasing sequence $\left\langle k_{n}<\omega: n<\omega\right\rangle$ such that for every sequence $\left\langle J_{n} \subseteq \tau: n<\omega\right\rangle$, where each $J_{n}$ is an cover of $[\mathcal{X}]^{k_{n}}$, there is a sequence $\left\langle D_{n} \in J_{n}: n<\omega\right\rangle$ with $\mathcal{X} \subseteq \bigcup_{n<\omega} \bigcap_{m>n} D_{m}$.

It is proved in [18] that every strong $\gamma$-set $X \subseteq \mathbb{R}$ is strongly meager. The goal of this section is to construct, under $\mathrm{CPA}_{\text {cube }}^{\text {game }}$, an uncountable strong $\gamma$-set in $\mathcal{P}(\omega)$. So, after indentifying $\mathcal{P}(\omega)$ with its homeomorphic copy in $\mathbb{R}$, this will be uncountable $\gamma$-set in $\mathbb{R}$ which is strongly meager. Under Martin's axiom such a strong $\gamma$-set of cardinality continuum was constructed in [18].

Let $\mathcal{B}_{0}$ be a countable basis for the topology of $\mathcal{P}(\omega)$ and let $\mathcal{B}$ be the collection of all finite unions of elements from $\mathcal{B}_{0}$. Since every open cover of $[\mathcal{P}(\omega)]^{k}, k<\omega$, contains a refinament from $\mathcal{B}$, in the definition of strong $\gamma$-set it is enough to consider only sequences $\left\langle J_{n}: n<\omega\right\rangle$ with $J_{n} \subseteq \mathcal{B}$.

Now, consider $\mathcal{B}$ with the discrete topology. Since $\mathcal{B}$ is countable, the space $\mathcal{B}^{\omega}$, considered with the product topology, is a Polish space and so is 
$\mathcal{X}=\left(\mathcal{B}^{\omega}\right)^{\omega}$. For $J \in \mathcal{X}$ we will write $J_{n}$ instead instead of $J(n)$. It is easy to see that a subbasis for the topology of $\mathcal{X}$ is given by the clopen sets:

$$
\left\{J \in \mathcal{X}: J_{n}(m)=B\right\},
$$

where $n, m<\omega$ and $B \in \mathcal{B}$.

For the reminder of this section fix an increasing sequence $\left\langle k_{n}: n<\omega\right\rangle$ such that $k_{n} \geq n 2^{n}+n$ for every $n<\omega$. Then we have the following lemma.

Lemma 2.1 Let $X \in[\omega]^{\omega}$ and let $F$ a countable subset of $\mathcal{P}(\omega)$ such that $[\omega]^{<\omega} \subseteq F$. Assume that $P$ is a compact subset of $\mathcal{X}$ such that for every $J \in P$ and $n<\omega$ the family $J_{n}[\omega]=\left\{J_{n}(m): m<\omega\right\}$ covers $[F]^{k_{n}}$. Then, there exists a set $Y \in[X]^{\omega}$ and for each $J \in P$ a sequence $\left\langle D_{n}^{J} \in J_{n}: n<\omega\right\rangle$ such that $F \cup Y^{*} \subseteq \bigcup_{n<\omega} \bigcap_{m>n} D_{m}^{J}$.

Proof. Let $\left\{F_{n}: n<\omega\right\}$ be an enumeration of $[\omega]^{<\omega}$ such that $F_{n} \subseteq n$ for all $n<\omega$ and let $F=\left\{f_{n}: n<\omega\right\}$. We will construct inductively the sequences $\left\langle s_{n} \in X: n<\omega\right\rangle$ and $\left\langle\left\{D_{n}^{J} \in J_{n}[\omega]: J \in P\right\}: n<\omega\right\rangle$ such that for every $n<\omega, J \in P$ and $A \subseteq \omega$ we have

(i) $\left\{f_{i}: i<n\right\} \subseteq D_{n}^{J}$ and $s_{n}<s_{n+1}$;

(ii) if $i<j \leq n+1$ and $A \cap s_{n+1}=F_{i}$ then $A \in D_{j}^{J}$.

We choose $s_{0} \in X$ and $\left\{D_{n}^{J} \in J_{n}[\omega]: J \in P\right\}$ arbitrarily. Then conditions (i) and (ii) are trivially satisfied. Next, assume that the sequence $\left\{s_{i}: i \leq n\right\}$ is already constructed. We will construct $s_{n+1}$ and sets $D_{n+1}^{J}$ as follows.

Let

$$
Q=\left\{q \in[\omega]^{<\omega}: q \backslash\left\{s_{0}, \ldots, s_{n}\right\}=F_{i} \text { for some } i \leq n\right\} .
$$

Then $|Q| \leq(n+1) 2^{n+1}$ and $\left|Q \cap\left\{f_{0}, \ldots, f_{n}\right\}\right| \leq k_{n+1}$.

Fix $J \in P$. Since $J_{n}[\omega]$ covers $[F]^{\leq k_{n+1}}$, there exists a $\bar{D}_{n+1}^{J} \in J_{n+1}[\omega]$ containing $Q \cup\left\{f_{0}, \ldots, f_{n}\right\}$. Since $\bar{D}_{n+1}^{J}$ is open and covers the finite set $Q$, there is an $s_{n+1}^{J}>s_{n}$ in $\mathcal{X}$ such that for every $q \in Q$

$$
\left\{x \subseteq \omega: x \cap s_{n+1}^{J}=q \cap s_{n+1}^{J}\right\} \subseteq \bar{D}_{n+1}^{J} .
$$

Notice that

$$
\text { for every } A \subseteq \omega \text { and } \bar{s}_{n+1} \geq s_{n+1}^{J} \text { condition (ii) holds. }
$$


Indeed, assume that $\left(A \cap \bar{s}_{n+1}\right) \backslash\left\{s_{0}, \ldots, s_{n}\right\}=F_{i}$ for some $i<j \leq n+1$. If $j \leq n$ then $n \geq 1$ and since $F_{i} \subseteq i \subseteq s_{n-1}$ we have

$$
\left(A \cap s_{n}\right) \backslash\left\{s_{0}, \ldots, s_{n-1}\right\}=\left(A \cap s_{n}\right) \backslash\left\{s_{0}, \ldots, \bar{s}_{n-1}\right\}=F_{i} .
$$

By the inductive assumption, $A \in D_{j}^{J}$. If $j=n+1$ then $q=A \cap \bar{s}_{n+1} \in Q$. So $A \in\left\{x \subseteq \omega: x \cap \bar{s}_{n+1}=q \cap \bar{s}_{n+1}\right\} \subseteq\left\{x \subseteq \omega: x \cap s_{n+1}^{J}=x \cap s_{n+1}^{J}\right\} \subseteq \bar{D}_{n+1}^{J}$, finishing the proof of $(\star)$.

For each $J \in P$ let $m^{J}<\omega$ be such that $J_{n+1}\left(m^{J}\right)=\bar{D}_{n+1}^{J}$ and define $U_{J}=\left\{K \in \mathcal{X}: K_{n+1}\left(m^{J}\right)=\bar{D}_{n+1}^{J}\right\}$. Then $U_{J}$ is an open neighborhood of $J$. In particular $\left\{U_{J}: J \in P\right\}$ is an open cover of the compact set $P$, so there exists $P_{0} \in[P]^{<\omega}$ such that $P \subseteq \bigcup\left\{U_{\bar{J}}: \bar{J} \in P_{0}\right\}$. Choose $s_{n+1} \in X$ such that $s_{n+1} \geq \max \left\{s_{n+1}^{\bar{J}}: \bar{J} \in P_{0}\right\}$. Moreover, for every $J \in P$ choose $\bar{J} \in P_{0}$ such that $j \in U_{\bar{J}}$ and define $D_{n+1}^{J}=\bar{D}_{n+1}^{\bar{J}}$. It is easy to see that, by $(\star)$, conditions (i) and (ii) are preserved. This completes the inductive construction.

Put $Y=\left\{s_{n}: n<\omega\right\}$. To see that it satisfies the lemma pick an arbitrary $J \in P$. We will show that $F \cup Y^{*} \subseteq \bigcup_{n<\omega} \bigcap_{m>n} D_{m}^{J}$.

Clearly $F \subseteq \bigcup_{n<\omega} \bigcap_{m>n} D_{m}^{J}$ since, by (i), $f_{n} \in D_{m}^{J}$ for every $m>n$. So, fix an $A \in Y^{*}$. Then, $A \backslash Y=F_{i}$ for some $i<\omega$. Let $n<\omega$ be such that $i<n$ and $s_{n}>\max \left(F_{i}\right)$. Then, for every $m>n$ we have $i<m<m+1$ and $\left(A \cap s_{m+1}\right) \backslash\left\{s_{0}, \ldots, s_{m}\right\}=F_{i}$. So, by (ii), we have $A \in D_{m+1}^{J}$ for every $m>n$. Thus, $A \in \bigcap_{m>n} D_{m}^{J}$.

Lemma 2.2 If $F \subseteq \mathcal{P}(\omega)$ is countable, then the set

$$
\mathcal{X}_{F}=\left\{J \in \mathcal{X}: J_{n}[\omega] \text { covers }[F]^{k_{n}} \text { for every } n<\omega\right\}
$$

is Borel in $\mathcal{X}$.

Proof. This follows from the fact that

$$
\mathcal{X}_{F}=\bigcap_{n<\omega} \bigcap_{A \in[F]^{k_{n}}} \bigcup_{m<\omega} \bigcup_{A \subseteq B \in \mathcal{B}}\left\{J \in \mathcal{X}: J_{n}(m)=B\right\}
$$

since each set $\left\{J \in \mathcal{X}: J_{n}(m)=B\right\}$ is clopen in $\mathcal{X}$. Thus, $\mathcal{X}_{F}$ is a $G_{\delta}$-set.

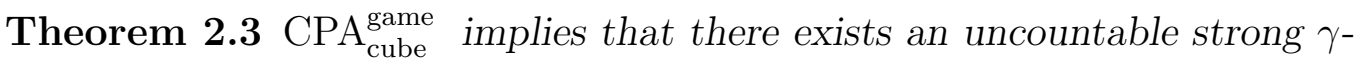
set in $\mathcal{P}(\omega)$. 
Proof. For $\alpha<\omega_{1}$ and an $\subseteq^{*}$-decreasing sequence $\mathcal{V}=\left\{V_{\xi} \in[\omega]^{\omega}: \xi<\alpha\right\}$ let $W(\mathcal{V}) \in[\omega]^{\omega}$ be such that $W(\mathcal{V}) \subsetneq^{*} V_{\xi}$ for all $\xi<\alpha$. Moreover, if $P \in \operatorname{Perf}^{*}(\mathcal{X})$ is a cube, then we define a subcube $Q=Q(\mathcal{V}, P)$ of $P$ and an infinite subset $Y=V(\mathcal{V}, P)$ of $X=W(\mathcal{V})$ as follows. Let $F=\mathcal{V} \cup[\omega]^{<\omega}$ and choose a subcube $Q$ of $P$ such that either $Q \cap \mathcal{X}_{F}=\emptyset$ or $Q \subseteq \mathcal{X}_{F}$. This can be done by 1.2 since $\mathcal{X}_{F}$ is Borel. If $Q \cap \mathcal{X}_{F}=\emptyset$ we put $Y=X$. Otherwise apply Lemma 2.1 to find $Y$.

Consider the following strategy $S$ for Player II:

$$
S\left(\left\langle\left\langle P_{\eta}, Q_{\eta}\right\rangle: \eta<\xi\right\rangle, P_{\xi}\right)=Q\left(\left\{V_{\eta}: \eta<\xi\right\}, P_{\xi}\right),
$$

where sets $V_{\eta}$ are defined inductively by $V_{\eta}=V\left(\left\{V_{\zeta}: \zeta<\eta\right\}, P_{\eta}\right)$. By $\mathrm{CPA}_{\text {cube }}^{\text {game }}$ strategy $S$ is not a winning strategy for Player II. So there is a game $\left\langle\left\langle P_{\xi}, Q_{\xi}\right\rangle: \xi<\omega_{1}\right\rangle$ played according $S$ in which Player II loses, that is, $\mathcal{X}=\bigcup_{\xi<\omega_{1}} Q_{\xi}$. Let $\mathcal{V}=\left\{V_{\xi}: \xi<\omega_{1}\right\}$ be a sequence associated with this game, which is strictly $\subsetneq^{*}$-decreasing, and let $T=\mathcal{V} \cup[\omega]^{<\omega}$. We claim that $T$ is a strong $\gamma$-set.

Indeed, let $\left\langle\mathcal{U}_{n} \subseteq \mathcal{B}: n<\omega\right\rangle$ be such that $\mathcal{U}_{n}$ covers $[T]^{k_{n}}$ for every $n<\omega$. Let $\alpha<\omega_{1}$ be such that $J \in Q_{\alpha}$. Then $J \in \mathcal{X}_{[\omega]<\omega \cup\left\{V_{\eta}: \eta<\alpha\right\}}$, so we must have used Lemma 2.1 to get the subcube $Q_{\alpha}$. In particular, there is a sequence $\left\langle D_{n}^{J} \in J_{n}[\omega]=\mathcal{U}_{n}: n<\omega\right\rangle$ such that $\left([\omega]^{<\omega} \cup\left\{V_{\eta}: \eta<\alpha\right\}\right) \cup\left(V_{\alpha}\right)^{*}$ is contained in $\bigcup_{n<\omega} \bigcap_{m>n} D_{m}^{J}$. Therefore, $T \subseteq \bigcup_{n<\omega} \bigcap_{m>n} D_{m}^{J}$, whenever $\left\{V_{\eta}: \alpha \leq \eta<\omega_{1}\right\} \subseteq\left(V_{\alpha}\right)^{*}$.

Since every homemorphic image of a strong $\gamma$-set is evidently a strong $\gamma$-set, we can conclude immediately the following.

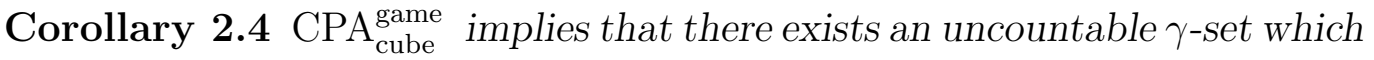
is strongly meager.

It is worth mentioning that a construction of an uncountable $\gamma$-set in $\mathcal{P}(\omega)$ under axiom $\mathrm{CPA}_{\text {cube }}^{\text {game }}$ can also be obtained by using an approachn similar to that used in [41]. In order to do this, we need the following definitions and facts. for a fixed sequence $\bar{k}=\left\langle k_{n} \in \omega: n<\omega\right\rangle$ we say that a family $\mathcal{A} \subseteq(\mathcal{P}(\omega))^{\omega}$ is $\bar{k}$-centered provided for every $n<\omega$ any $k_{n}$-many sets from $\{A(n): A \in \mathcal{A}\}$ has a common point and $B \subseteq \omega^{\omega}$ is a quasi-intersection of $\mathcal{A} \subseteq(\mathcal{P}(\omega))^{\omega}$ provided for every $A \in \mathcal{A}$ for all but infinitely-many $n<\omega$ we have $B(n) \in A(n)$. Now, if $\mathcal{K}^{*}$ is the family of all continuous from $\mathcal{P}(\omega)$ to $(\mathcal{P}(\omega))^{\omega}$, then, the following is true: 
A set $X \subseteq \mathcal{P}(\omega)$ is a strong $\gamma$-set if and only if there exists an increasing sequence $\bar{k}=\left\langle k_{n} \in \omega: n\langle\omega\rangle\right.$ such that for every $f \in \mathcal{K}^{*}$, if $f[X]$ is $\bar{k}$ centered, then $f[X]$ has a quasi-intersection.

With this characterization in hand we can construct an uncountable strong $\gamma$-set in $\mathcal{P}(\omega)$ by applying $\mathrm{CPA}_{\text {cube }}^{\text {game }}$ to the Polish space $\mathcal{K}^{*}$. 


\section{Chapter 3}

\section{Ultrafilters on $\mathbb{Q}$}

In this chapter symbol $X$ will represent a countably infinite set.

\subsection{Preliminaries and introduction}

\subsubsection{Ideals, filters, and ultrafilters on $X$}

A (non-trivial) ideal on $X$ is a non-empty family $\mathcal{I} \subseteq \mathcal{P}(X)$ such that

(I1) $X \notin \mathcal{I}$,

(I2) $A \cup B \in \mathcal{I}$ provided $A, B$ are in $\mathcal{I}$, and

(I3) if $A \subseteq B$ and $B \in \mathcal{I}$, then $A \in \mathcal{I}$.

The dual notion of an ideal on $X$ is that of a filter on $X$. A (non-trivial) filter on $X$ is a family $\mathcal{F} \subseteq \mathcal{P}(X)$ such that

(F1) $\emptyset \notin \mathcal{F}$

(F2) $A \cap B \in \mathcal{F}$ provided $A, B$ are in $\mathcal{F}$, and

(F3) if $A \subseteq B$ and $A \in \mathcal{F}$, then $B \in \mathcal{F}$.

The duality between these two notions becomes evident by the fact that if $\mathcal{I}$ is an ideal on $X$, the corresponding dual filter of $\mathcal{I}$ is the set

$$
\mathcal{F}_{\mathcal{I}}=\{X \backslash A: A \in \mathcal{I}\} .
$$


Similarly, given any filter $\mathcal{F}$ on $X$, the corresponding dual ideal is the set

$$
\mathcal{I}_{\mathcal{F}}=\{X \backslash A: A \in \mathcal{F}\} .
$$

If $\mathcal{I} \subseteq \mathcal{P}(X)$ is an ideal, we will consider the family of $\mathcal{I}$-positive subsets of $X$ defined by $\mathcal{I}^{+}=\mathcal{P}(X) \backslash \mathcal{I}$.

The set $[X]^{<\omega}$ of finite subsets of $X$ is an ideal that we will denote $\mathcal{I}_{\text {fin }}$. Its dual filter $\mathcal{F}_{\text {cofin }}$ is the family of the complements of the finite sets and it is called the cofinite filter or Fréchet filter.

The main subject of this section is that of ultrafilter on $X$. An ultrafilter $\mathcal{U}$ on $X$ is a filter on $X$ which is not strictly contained in any other filter on $X$. This is, $\mathcal{U}$ is maximal with respect to $\subseteq$. Its dual notion is that of prime ideal; however, all our concerns will be always directed to ultrafilters on some suitable $X$. The maximality condition on an ultrafilter $\mathcal{U}$ implies, in particular, that if $F \in[\mathcal{U}]^{<\omega}$ and $\bigcup F \in \mathcal{U}$, then $F \cap \mathcal{U} \neq \emptyset$. The following proposition provides a technique of constructing ultrafilters in $\mathrm{ZFC}$.

Proposition 3.1 If $S$ is any infinte set and $\mathcal{F} \subseteq \mathcal{P}(X)$ has the finite intersection property, then $\mathcal{F}$ can be extended to an ultrafilter on $S$.

Ultrafilters on a fixed $X$ can be classified into principal or fixed ultrafilters and non-principal or free ultrafilters. The first are generated by a singleton in $X$. That means that if $\mathcal{U} \subseteq \mathcal{P}(X)$ is a principal ultrafilter, then there exists an $x \in X$ such that $\mathcal{U}=\{A \subseteq X: x \in A\}$. Therefore, we can identify any principal ultrafilter with the singleton that generates it and the set $X$ with the set of principal ultrafilters on it. It is also easy to see that an ultrafilter $\mathcal{U}$ on $X$ is principal if and only if $\mathcal{U} \cap \mathcal{I}_{\text {fin }} \neq \emptyset$ and that $\mathcal{U}$ is non-principal if and only if $\mathcal{F}_{\text {cofin }} \subseteq \mathcal{U}$.

Given a non-principal ultrafilter $\mathcal{U}$ on $X$ we say that $\mathcal{B} \subseteq \mathcal{U}$ is a base for $\mathcal{U}$ if every member of $\mathcal{U}$ contains a member in $\mathcal{B}$. The character of $\mathcal{U}$ is the cardinal number defined by

$$
\chi(\mathcal{U})=\min \{|\mathcal{B}|: \mathcal{B} \text { is a base for } \mathcal{U}\} .
$$

It is an easy exercise to show that $\chi(\mathcal{U})$ is always an uncountable cardinal.

\subsubsection{The spaces $\beta \omega$ and $\omega^{*}$}

We will give a structure of topological space to the set

$$
\beta \omega=\{\mathcal{U}: \mathcal{U} \text { is an ultrafilter on } \omega\}
$$


by considering the family $\{V(A): A \in \mathcal{P}(\omega)\}$ as a basis for the topology on $\beta \omega$, where $V(A)=\{\mathcal{U} \in \beta \omega: A \in \mathcal{U}\}$ for every $A \in \mathcal{P}(\omega)$. The sets $V(A)$ have the following properties:

(a) $V(\emptyset)=\emptyset$ and $V(\omega)=\beta \omega$,

(b) $V(\omega \backslash A)=\beta \omega \backslash V(A)$,

(c) $V(A \cap B)=V(A) \cap V(B)$ for every $A, B \in \mathcal{P}(\omega)$, and

(d) $V(A \cup B)=V(A) \cup V(B)$ for every $A, B \in \mathcal{P}(\omega)$.

Property (c) indicates that the family $\{V(A): A \in \mathcal{P}(\omega)\}$ is a base for a topology $\tau$ on $\beta \omega$. This topology makes the pair $\langle\beta \omega, \tau\rangle$ a compact, Hausdorff space containing a homeomorphic copy of the discrete space $\omega$. It turns out that $\langle\beta \omega, \tau\rangle$ is homemorphic to the Stone-Čech compactification of $\omega$. Since (the homeomorphic copy of) $\omega$ is open in $\beta \omega$ it turns out that the remainder $\omega^{*}=\beta \omega \backslash \omega$ is also a compact Hausdorff space which is homeomorphic to Stone space of the Boolean algebra $\mathcal{P}(\omega) / \mathcal{I}_{\text {fin }}$.

The literature about remainders of Stone-Cech compactifications is enormous. Among the many problems arising from this subject a great deal of effort has been dedicated to solve the general problem of its homogeneity. Recall that a topological space $\langle S, \tau\rangle$ is homogeneous provided for any two points $x, y \in S$ there exists an autohomeomorphism $h: S \rightarrow S$ such that $h(x)=y$. Very informally, this means that we cannot distinguish points in $\langle S, \tau\rangle$. All of them have the same topological behavior ${ }^{1}$. A natural problem in topology is to find out what properties of a space $S$ transfer to $S^{*}$. For example, is $S^{*}$ always homogeneous when $S$ is? The negative answer to this general question was provided by $\mathrm{W}$. Rudin in [44]. In that paper he produced, under $\mathrm{CH}$, two points in $\omega^{*}$ that behave very differently.Therefore, there could not be a autohomeomorphism of $\omega^{*}$ carrying one onto the other.

\subsubsection{Special points in $X^{*}$}

Given a topological space $\langle S, \tau\rangle$, we say that an $x \in S$ is a $P$-point if the intersection of countably-many neighborhoods of $x$ contains a neighborhood of $x$. If we take a countably infinite set $X$ with the discrete topology, then

\footnotetext{
${ }^{1}$ The interval $(0,1)$, the Cantor space $2^{\omega}$, and the Hilbert cube, for example, are homogeneous spaces; the interval $(0,1]$ is not.
} 
$X^{*}$ is homeomorphic to $\omega^{*}$ and $P$-points can be defined in a purely combinatorial fashion. Since we are mainly concerned with the ultrafilters on $X$ as combinatorial entities, we will concentrate on this aspect when we dicuss their properties and barely mention its topological translation.

Definition 3.1 A non-principal ultrafilter $\mathcal{U}$ on $X$ is a $P$-point if for every partition $\mathcal{P}$ of $X$ such that $\mathcal{U} \cap \mathcal{P} \neq \emptyset$ there exists an $X \in \mathcal{U}$ such that $X \cap P$ is finite for each $P \in \mathcal{P}$.

In his paper [44] W.Rudin used $\mathrm{CH}$ to construct a $P$-point on $\omega$. Since in ZFC there is always a non- $P$-point ${ }^{2}$ this proves the non-homogeneity of $\omega^{*}$ because no homeomorphism $h: \omega^{*} \rightarrow \omega^{*}$ carries a $P$-point onto a non- $P$ point.

Although Rudin's proof exhibited two points in $\omega^{*}$ that behave differently his proof was considered "dishonest" since it used $\mathrm{CH}$, which is unprovable from ZFC.

In [17] Z. Frolík gave a ZFC proof of the non-homogeneity of $\omega^{*}$. This proof is not "honest" because it does not show why $\omega^{*}$ is not homogeneous. The first "honest" proof of the non-homogenity of $\omega^{*}$ was provided by Kunen in [27]. The idea behind Kunen's proof is the same as Rudin's. The important difference is that Kunen was able to produce in ZFC a different kind of singular point, namely a weak $P$-point.

Definition 3.2 If $\langle S, \tau\rangle$ is a topological space, an $x \in S$ is a weak $P$-point if $x$ is not in the closure of any countable subset of $S$.

It is easy to show that there always exist points in $X^{*}$ that are not weak $P$-points. Working in $\omega$, let $\left\{\mathcal{U}_{n}: n<\omega\right\}$ be a countable family of nonprincipal ultrafilters on $\omega$ and let $\mathcal{V}$ be any non-principal ultrafilter on $\omega$. Consider $\mathcal{W} \subseteq \mathcal{P}(\omega)$ given by

$$
A \in \mathcal{W} \leftrightarrow\left\{n<\omega: A \in \mathcal{U}_{n}\right\} \in \mathcal{V}
$$

By using the definition of basic sets in $\omega^{*}$ it is immediate that $\mathcal{W}$ is a nonprincipal ultrafilter and that $\mathcal{W} \in \overline{\left\{\mathcal{U}_{n}: n<\omega\right\}}$. A completely different situation is to show in ZFC that there exist weak $P$-points. In order to do this Kunen introduced the notion of a $\kappa$-OK point for any infinite cardinal $\kappa$.

\footnotetext{
${ }^{2}$ If $\mathcal{P}=\left\{P_{n}: n<\omega\right\}$ is a partition of $\omega$ into infinite pieces consider the filter given by $\mathcal{F}=\left\{A \subseteq \omega: \forall^{\infty} n<\omega P_{n} \subseteq A\right\}$. Then, no ultrafilter $\mathcal{U}$ extending $\mathcal{F}$ can be a $P$-point.
} 
Definition 3.3 Let $\kappa$ be an infinite cardinal and let $\mathcal{U}$ be a non-principal ultrafilter on $X$. We say that $\mathcal{U}$ is a $\kappa-O K$ point provided that for every $\left\langle V_{n} \in \mathcal{U}: n<\omega\right\rangle$ there exists a $\left\langle U_{\xi} \in \mathcal{U}: \xi<\kappa\right\rangle$ such that for every $n<\omega$ and $\xi_{0}<\cdots<\xi_{n}$

$$
\bigcap_{i \leq n} U_{\xi_{i}} \subseteq^{*} V_{n}
$$

Ordinary ultrafilters are $\omega$-OK points and the condition of being a $\kappa$ OK point gets stronger as $\kappa$ increases. The next two propositions solve the homegeneity problem in ZFC for $\omega^{*}$.

Proposition 3.2 (Kunen [27]) Every $P$-point is a $\kappa$-OK point for every $\kappa$ and every $\omega_{1}$-OK point is a weak $P$-point.

Proposition 3.3 (Kunen [27]) There are $\mathfrak{c}-O K$ points in $\omega^{*}$. Moreover, there are $2^{\mathfrak{c}}$-many of these ultrafilters and they can be made of character $\mathfrak{c}$.

Although Kunen's proof solved the homogeneity problem for $\omega^{*}$ the question wether $P$-point exists in ZFC remained open for many years. A negative solution to this question was provided by S. Shelah who found a model of ZFC without $P$-points [45].

Other ultrafilters relevant to this work are $Q$-points and selective ultrafilters.

Definition 3.4 A non-principal ultrafilter $\mathcal{U}$ on $X$ is called a $Q$-point on $X$ provided that for every infinite partition $\mathcal{P}$ of $X$ into finite sets either $\mathcal{U} \cap \mathcal{P} \neq \emptyset$ or there exists a $U \in \mathcal{U}$ such that $|U \cap P| \leq 1$ for every $P \in \mathcal{P}$. Such an $U$ is called a partial selector of $\mathcal{P}$.

Definition 3.5 A non-principal ultrafilter $\mathcal{U}$ on $X$ is called selective on $X$ provided that for every infinite partition $\mathcal{P}$ of $X$ either $\mathcal{U} \cap \mathcal{P} \neq \emptyset$ or $\mathcal{U}$ contains a partial selector of $\mathcal{P}$.

It is easy to check that a non-pricipal ultrafilter $U$ on $X$ is selective ${ }^{3}$ if and only if it is simultaneously a $P$-point and a $Q$-point. As in the case of $P$-points the existence of $Q$-points and selective ultrafilters follows from $\mathrm{CH}$ and Martin's axiom.

Some years before Shelah proved the consistency of non-existence of $P$ points K. Kunen and A. Miller proved, respectively, the following theorems.

\footnotetext{
${ }^{3}$ Selective ultrafilters are also called Ramsey in the literature.
} 
Proposition 3.4 (Kunen [29]) Let $M$ be a transitive model of ZFC plus $G C H$. There is a generic extension of $M$ in which there are no selective ultrafilters.

Proposition 3.5 (Miller [37]) There are not Q-points in Laver's model of ZFC for the Borel Conjecture.

Consider $\mathbb{Q}$ with the subspace topology inherited from the usual topology on $\mathbb{R}$. A set $B \subseteq \mathbb{Q}$ is scattered if every non-empty subset of $B$ has isolated points. It is easy to see that the scattered subsets of $\mathbb{Q}$ form an ideal, which we will denote by $\mathcal{I}_{S}$.

The following facts will be used in what follows. For the proofs see [9] or [10, Fact 5.5.1].

Fact 3.6 (K. Ciesielski, J. Pawlikowski $[9,10]$ ) Every non-scattered set $B \subseteq$ $\mathbb{Q}$ contains a subset from $\operatorname{Perf}(\mathbb{Q})$.

Let $\mathcal{J}$ be an ideal on a countable set $X$. Then we define $\mathcal{J}^{+}=\mathcal{P}(X) \backslash \mathcal{J}$. We say that $\mathcal{J}$ is weakly selective if for every $A \in \mathcal{J}^{+}$and $f: A \rightarrow X$ there exists a $B \in \mathcal{P}(A) \cap \mathcal{J}^{+}$such that $f\lceil B$ is either one-to-one or constant.

Fact 3.7 (K. Ciesielski, J. Pawlikowski $[9,10]$ ) The ideals $[\omega]^{<\omega}$ and $\mathcal{I}_{S}$ are weakly selective.

An ultrafilter $\mathcal{U}$ on $\mathbb{Q}$ is crowded provided it contains a basis consisting of perfect sets. These ultrafilters were considered by E. K. van Douwen as examples of particularly nice points in $\mathbb{Q}^{*}$. He proved that they exist under Martin's Axiom in [14]. This result has been generalizad by Coplakova and Hart in [13]. In that paper they show that the existence of a crowded ultrafilter follows from the equality $\mathfrak{b}=\mathfrak{c}$. Is is well known that it holds in Laver's model for Borel's conjecture where all ultrafilters have character c. In [9] Ciesielski and Pawlikowski showed that under $\mathrm{CPA}_{\text {prism }}^{\text {game }}$ crowded ultrafilters of character $\omega_{1}$ exist. To construct their example Ciesielski and Pawlikowski made use of the following result. A proof can be found in $[9$, lem. 4.9(b)] or in $\left[10\right.$, lem. 5.3.4(b)], where $[X]^{\omega}$ comes with a subspace topology of $\mathcal{P}(X)$, with $\mathcal{P}(X)$ being identified with $2^{X}$ via characteristic function.

Proposition 3.8 (K. Ciesielski, J. Pawlikowski $[9,10]$ ) Let $X$ be countably infinite and let $\mathcal{J} \subseteq \mathcal{P}(X)$ be a weakly selective ideal. For every prism $P \subseteq$ $[X]^{\omega}$ and every $A \in \mathcal{J}^{+}$there exist a subprism $Q$ of $P$, a $B \in \mathcal{P}(A) \cap \mathcal{J}^{+}$, and an $i<2$ such that $g \uparrow B$ is constant equal to $i$ for every $g \in Q$. 


\begin{tabular}{|c|c|c|c|c|}
\hline$P$-point & $Q$-point & $\omega_{1}$-OK point & Existence & Reference \\
\hline- & - & - & under CPA $_{\text {prism }}^{\text {game }}$ & Theorem 3.18 \\
\hline- & - & + & under CPA $_{\text {prism }}^{\text {game }}$ & Theorem 3.35 \\
\hline- & + & - & under CPA $_{\text {prism }}^{\text {game }}$ & Corollary 3.22 \\
\hline- & + & + & under CPA $_{\text {prism }}^{\text {game }}$ & Corollary 3.37 \\
\hline+ & - & - & No, in ZFC & Proposition 3.2 \\
\hline+ & - & + & under CPA \\
\hline prism & {$[9]$ or [10] } \\
\hline+ & + & - & No, in ZFC & Proposition 3.2 \\
\hline+ & + & + & under CPA \\
\hline prism & {$[9]$ or [10] } \\
\hline
\end{tabular}

Table 3.1: Existence of different ultrafilters. All constructed ultrafilters are non-principal and $\omega_{1}$-generated. Moreover, the first four examples can be made also crowded.

\subsection{Main results}

In this chapter we establish, under $\mathrm{CPA}_{\text {prism }}^{\text {game }}$, the existence of a nonselective $Q$-point (i.e., a $Q$-point which is not a $P$-point) by constructing an $\omega_{1}$ generated crowded $Q$-point which is also an $\omega_{1}$-OK point (Corollary 3.37). This improves a previous construction of ours [7] of an $\omega_{1}$-generated crowded $Q$-point on $\mathbb{Q}$. We also prove, under $\mathrm{CPA}_{\text {prism }}^{\text {game }}$, that there exist crowded $\omega_{1}$-generated $Q$-points that are not $\omega_{1}$-OK points (Corollary 3.22), crowded $\omega_{1}$-generated $\omega_{1}$-OK points which are neither $P$-points nor $Q$-points (Theorem 3.35), and crowded $\omega_{1}$-generated ultrafilters on $\omega$ that are neither $Q$ points nor $\omega_{1}$-OK points (Theorem 3.18). These complete all the logical implications between being a $P$-point, a $Q$-point, or an $\omega_{1}$-OK point as Table 3.1 shows.

Besides the properties explicitly listed in Table 3.1 we consider also two other properties: being $\omega_{1}$-generated (with $\omega_{1}<\mathfrak{c}$ ) and being crowded.

As mentioned above, the first four examples from Table 3.1 are also crowded. On the other hand that no other example from Table 3.1 can be crowded, since a crowded ultrafilter cannot be a $P$-point [9, prop. 4.25]. It is also easy to see that we can destroy the property of being crowded without changing any of the remaining properties. To see this, note that if $\mathcal{U}$ is an ultrafilter on $\mathbb{Q}$ and $f$ is a bijection between $\mathbb{Q}$ and a scattered subset $S$ of $\mathbb{Q}$, then $\mathcal{V}=\left\{A \subseteq \mathbb{Q}: f^{-1}(A) \in \mathcal{U}\right\}$ is a noncrowded ultrafilter that has the remaining properties identical to that of $\mathcal{U}$. 
One of the key features of our examples is that they are all $\omega_{1}$-generated with $\omega_{1}<\mathfrak{c}$. This cannot be achieved in ZFC, since in many models of ZFC, for example under MA, every non-principal ultrafilter on a countable set has character $\mathfrak{c}$. On the other hand, every example cited in Table 3.1 can be constructed under MA if we are willing to settle for $\mathfrak{c}$-generated filters. An interesting issue is whether under $\mathrm{CPA}_{\text {prism }}^{\text {game }}$ the examples from Table 3.1 must be $\omega_{1}$-generated. The answer is positive for the last example from the table, since Ciesielski and Pawlikowski proved (see [9, cor. 2.7] or $\left[10\right.$, cor. 1.5.4]) that under $\mathrm{CPA}_{\text {prism }}^{\text {game }}$ every selective ultrafilter is $\omega_{1}$-generated. There is some indication suggesting that $\mathrm{CPA}_{\text {prism }}^{\text {game }}$ implies that every $P$-point is $\omega_{1}$-generated. This would take care of the bottom half of the table. In the last section of this chapter we present the construction, under $\mathrm{CPA}_{\text {prism }}^{\text {game }}$, of many crowded $Q$-points of character $\mathfrak{c}$. These particular examples are not weak $P$-points so they cannot be $\omega_{1}$-OK points (See Proposition 3.2). The existence of an example of character $\mathfrak{c}$ as in the fourth row in the table is left open. The first two examples from Table 3.1 do not need to be $\omega_{1}$-generated. By Proposition 3.16 the Fubini product $\mathcal{U} \otimes \mathcal{U}$, where $\mathcal{U}$ is a Kunen's example from Proposition 3.3, is as the first ultrafilter from Table 3.1. The second of these is justified by a slight modification ${ }^{4}$ of Kunen's example from Proposition 3.3.

Finally, let us address a question, whether any of the examples from Table 3.1 can be constructed in ZFC. The answer is clearly no for all but the first two examples, since there are models of ZFC with no $P$-points (see [45]) as well as models of ZFC with no $Q$-points (see [37]). There are, however, a ZFC examples for the first two entries of Table 3.1 as mentioned above. These need not be $\omega_{1}$-generated, as we already noted. Whether they can be crowded remains unclear, since it is an open problem if there exists a crowded ultrafilter in ZFC.

\subsection{Some important lemmas}

Let $X$ be a countably infinite set. If $\mathcal{F} \subseteq[X]^{\omega}$ is nonempty, we say that $\mathcal{F}$ has the strong finite intersection property, SFIP, provided that $|\cap F|=\omega$ for every nonempty $F \in[\mathcal{F}]^{<\omega}$. The following is a very well known and easy

\footnotetext{
${ }^{4}$ Let $\mathcal{F}_{0}$ be the dual filter of the ideal $\mathcal{I}_{0}=\left\{A \subseteq \omega: \varlimsup_{n \rightarrow \infty}\left|A \cap P_{n}\right|<+\infty\right\}$, where $\left\{P_{n}: n<\omega\right\}$ is the partition of $\omega$ such that $P_{n}=\left\{m<\omega: 2^{n}-1 \leq m<2^{n+1}-1\right\}$. Construct a $\mathfrak{c}$ by $\mathfrak{c}$ independent linked family w.r.t $\mathcal{F}_{0}$ and follow the argument from [27].
} 
fact.

Lemma 3.9 If $\mathcal{F} \subseteq[X]^{\omega}$ is nonempty, countable, and has the SFIP, then there exists a $C(\mathcal{F}) \in[X]^{\omega}$ such that $C(\mathcal{F}) \subseteq^{*} B$ for every $B \in \mathcal{F}$.

Proof. If $\mathcal{F}$ is finite, we put $C(\mathcal{F})=\bigcap \mathcal{F}$; otherwise $\mathcal{F}=\left\{B_{n}: n<\omega\right\}$ and we can pick inductively $b_{n} \in \bigcap_{k \leq n} B_{k}$ such that $b_{n} \notin\left\{b_{k}: k<n\right\}$. The set $C(\mathcal{F})=\left\{b_{n}: n<\omega\right\}$ works.

Let $X$ be a countably infinite set. If the set $Z_{X}=[X]^{<\omega} \backslash\{\emptyset\}$ has the discrete topology then the product space $\mathcal{Z}_{X}=\left(Z_{X}\right)^{\omega}$ is a Polish space and the sets $U_{\langle n, a\rangle}=\{z \in \mathcal{Z}: z(n)=a\}$, where $a \in[\omega]^{<\omega}$ and $n<\omega$, constitute a subbasis for the product topology. Consider the set

$$
\mathcal{P}_{X}=\left\{z \in \mathcal{Z}_{X}:\{z(k): k<\omega\} \text { is a partition of } \omega\right\} \text {. }
$$

If $X=\omega$ we will drop the indexes, that is, $\mathcal{Z}=\mathcal{Z}_{\omega}$ and $\mathcal{P}=\mathcal{P}_{\omega}$.

Lemma 3.10 $\mathcal{P}_{X}$ is a $G_{\delta}$ subset of $\mathcal{Z}_{X}$. Therefore $\mathcal{P}_{X}$ is a Polish space with the relative topology inherited from $\mathcal{Z}_{X}$.

Proof. We can assume that $X=\omega$. If $A=\left\{z \in \mathcal{Z}: \bigcup_{n<\omega} z(n)=\omega\right\}$ and $B=\{z \in \mathcal{Z}:\{z(n): n<\omega\}$ is pairwise disjoint $\}$ then $\mathcal{P}=A \cap B$. The set $A$ is $G_{\delta}$ because $A=\bigcap_{k \in \omega} \bigcup_{n<\omega} \bigcup\left\{U_{\langle n, a\rangle}: a \in[\omega]^{<\omega} \& k \in a\right\}$. The set $B$ is $G_{\delta}$ since it can be written as $\bigcap_{m<n<\omega} \bigcup\left\{U_{\langle m, a\rangle} \cap U_{\langle n, b\rangle}: a \cap b=\emptyset\right\}$. Thus, $\mathcal{P}$ is $G_{\delta}$ in $\mathcal{Z}$.

Definition 3.6 Let $X$ be a countably infinite set and let $\mathcal{J} \subseteq \mathcal{P}(X)$ be an ideal on $X$ containing all the singletons. We say that $\mathcal{J}$ is $Q$-like provided that for every $A \in \mathcal{J}^{+}$there exists a countable family $\left\{A_{n} \in[A]^{\omega}: n<\omega\right\}$ such that no set $\left\{b_{n}: n<\omega\right\}$ belongs to $\mathcal{J}$ provided $b_{n} \in A_{n}$ for every $n<\omega$.

Lemma 3.11 Let $X$ be a countably infinite set, let $\mathcal{J}$ be a $Q$-like ideal on $X$ and let $\left\{A_{n} \in \mathcal{J}^{+}: n<\omega\right\}$ be arbitrary. If $P$ is a prism on $\mathcal{P}_{X}$, then there exist a subprism $Q$ of $P$ and $\left\{B_{n} \in \mathcal{P}\left(A_{n}\right) \cap \mathcal{J}^{+}: n<\omega\right\}$ such that $\left|\left(\bigcup_{n<\omega} B_{n}\right) \cap z(k)\right| \leq 1$ for every $z \in Q$ and $k<\omega$. Moreover, if $P$ is a cube then $Q$ can be chosen as a subcube of $P$. 
Proof. We can suppose that $X=\omega$. Let $\left\langle A_{n}^{m} \in\left[A_{n}\right]^{\omega}: m<\omega\right\rangle$ be the sequence associated to $A_{n}$ in the definition of $Q$-like for every $n<\omega$ and let $\Gamma: \omega \times \omega \rightarrow \omega$ be any bijection. Define a sequence $\left\langle A_{r}^{*}: r<\omega\right\rangle$ by $A_{r}^{*}=$ $A_{\Gamma(m, n)}^{*}=A_{n}^{m}$ provided $\Gamma(m, n)=r$.

Case (a): If $P=\{z\}$ then, define a sequence $\left\langle b_{r} \in \omega: r<\omega\right\rangle$ inductively such that $b_{r} \in A_{r}^{*} \backslash \bigcup\left\{z(k): k<\omega \& z(k) \cap\left\{b_{0}, \ldots, b_{r-1}\right\} \neq \emptyset\right\}$ for every $r<\omega$. This is possible because the $A_{r}^{*}$ is infinite and the set of forbidden points is an union of finitely-many finite sets. Put $B_{n}=\left\{b_{\Gamma(m, n)}: m<\omega\right\}$. Therefore, $B_{n} \in \mathcal{P}\left(A_{n}\right) \cap \mathcal{J}^{+}$for every $n<\omega$ and $\left|\left(\bigcup_{n<\omega} B_{n}\right) \cap z(k)\right| \leq 1$ for every $k<\omega$.

Case (b): If $P \in \operatorname{Perf}\left(\mathcal{P}_{\omega}\right)$, let $f$ be a witness function for $P$. By Remark 1.1 we can assume that $f$ acts from $\mathfrak{C}^{\alpha}$ onto $P$. Thus, $P$ is a cube. It is enough to find its subcube with the desired properties.

Let $\mu$ be the standard product probability measure on $\mathfrak{C}^{\alpha}$. We construct, by induction on $r<\omega$, a sequence $\left\langle K_{r}: r<\omega\right\rangle$ of open subsets of $\mathfrak{C}^{\alpha}$ and two sequences, $\left\langle b_{r} \in A_{r}^{*}: r<\omega\right\rangle$ and $\left\langle D_{r} \in[\omega]^{<\omega}: r<\omega\right\rangle$, such that for every $r<\omega:$

(i) $b_{r}>\max \left(\left\{b_{i}: i<r\right\} \cup \bigcup_{j<r} D_{j}\right)$,

(ii) $\mu\left(K_{r}\right) \geq 1-2^{-(r+2)}$, and

(iii) $f(h)(k) \subseteq D_{r}$ for every $h \in K_{r}$ and $k<\omega$ for which $b_{r} \in f(h)(k)$.

If this construction is possible, put $B_{n}=\left\{b_{\Gamma(m, n)}: m<\omega\right\}$ for every $n<\omega$ and $B=\left\{b_{r}: r<\omega\right\}$ and notice that $B=\bigcup_{n<\omega} B_{n}$. Then, clearly $B_{n} \in \mathcal{P}\left(A_{n}\right) \cap \mathcal{J}^{+}$since that $\mathcal{J}$ is $Q$-like and $b_{\Gamma(m, n)} \in A_{n}^{m}$ for every $m<\omega$. Condition (ii) implies that $\mu\left(\bigcap_{r<\omega} K_{r}\right) \geq \frac{1}{2}$. Hence, by Proposition 1.2, there exists a perfect cube $C \subseteq \bigcap_{r<\omega} K_{r}$. Then $Q=f[C]$ is a subcube of $P$ and the pair $\left\langle Q,\left\{B_{n}: n<\omega\right\}\right\rangle$ is as required. To see this, it is enough to show that $|z(k) \cap B| \leq 1$ for every $z \in Q$ and $k<\omega$. Let $z=f(h)$ for some $h \in C$. By conditions (i) and (iii), for every $b_{j} \in z(k)=f(h)(k)$ and $r>j$ we have that $b_{r} \notin z(k)$. Therefore, no two elements of $B$ are in the same $z(k)$ or, in other words, $|z(k) \cap B| \leq 1$ for every $k<\omega$.

Next, we show that the inductive construction is possible. Let $r<\omega$ be such that the appropriate $b_{i}, K_{i}$, and $D_{i}$ are already constructed for every $i<r$. We will construct $b_{r}, K_{r}$, and $D_{r}$ satisfying (i)-(iii). We pick an $b_{r}$ as an arbitrary element of $A_{r}^{*}$ satisfying condition (i). Next, we define $L=\left\{a \in[\omega]^{<\omega}: b_{r} \in a\right\}$ and note that $\left\{f^{-1}\left(U_{\langle t, a\rangle}\right):\langle t, a\rangle \in \omega \times L\right\}$ is a 
partition of $\mathfrak{C}^{\alpha}$ into clopen sets. Thus, we can find a finite set $S \subseteq \omega \times L$ such that $K_{r}=\bigcup\left\{f^{-1}\left(U_{\langle t, a\rangle}\right):\langle t, a\rangle \in S\right\}$ satisfies condition (ii). Let $D_{r}=$ $\bigcup\{a:\langle t, a\rangle \in S$ for some $t<\omega\}$. Then clearly, $D_{r}$ is finite. To see that it satisfies (iii), take an $h \in K_{r}$. Then $f(h) \in U_{\langle t, a\rangle}$ for some $\langle t, a\rangle \in S$. Let $k<\omega$ be such that $b_{r} \in f(h)(k)$. Since we have also $b_{r} \in a=f(h)(t)$, we conclude that $k=t$. So, $f(h)(k)=f(h)(t)=a \subseteq D_{r}$.

Definition 3.7 Let $X$ be a countably infinite set. We say that an ideal $\mathcal{J}$ on $X$ is prism-friendly provided that it contains all singletons and

(•) given a prism $P$ in $2^{X}$ and an $A \in \mathcal{J}^{+}$there exists a subprism $Q$ of $P$, a $B \in \mathcal{P}(A) \cap \mathcal{J}^{+}$, and an $i<2$ such that $g\lceil B$ is constant equal $i$ for every $g \in Q$.

Definition 3.8 Let $X$ be a countably infinite set. We say that an ideal $\mathcal{J}$ on $X$ is rich if it is prism-friendly and

(\#) given an $A \in \mathcal{J}^{+}$there exists a family $\mathcal{A} \subseteq \mathcal{P}(A) \cap \mathcal{J}^{+}$of cardinality $\mathfrak{c}$ which is almost disjoint, that is, such that $|A \cap B|<\omega$ for every distinct $A, B \in \mathcal{A}$.

Also, notice that, in ZFC, condition $(\bullet)$ does not imply condition (\#). Indeed, if $\mathcal{U}$ is a selective ultrafilter, then its dual ideal $\mathcal{I}_{\mathcal{U}}$ is weakly selective. So, see [10], $\mathcal{I}_{\mathcal{U}}$ is prism-friendly. However, $\mathcal{I}_{\mathcal{U}}^{+}=\mathcal{U}$ and no two members in $\mathcal{U}$ can be almost disjoint.

Lemma 3.12 The ideals $[\omega]^{<\omega}$ and $\mathcal{I}_{S}$ are $Q$-like and rich.

Proof. It is easy to see that $[\omega]^{<\omega}$ is $Q$-like. To see that $\mathcal{I}_{S}$ is also $Q$ like pick any $A \in \mathcal{I}_{S}^{+}$. By Fact 3.7 we can assume that $A \in \operatorname{Perf}(\mathbb{Q})$. Let $\mathcal{B}$ be a countable basis for the topology on $\mathbb{Q}$ and let $\left\{A_{n}: n<\omega\right\}$ be an enumeration of the set $\{S \cap A: S \in \mathcal{B} \&|S \cap A|=\omega\}$. If $b_{n} \in A_{n}$ for every $n<\omega$ then $B=\left\{b_{n}: n<\omega\right\}$ is dense in $A$ and in consequence, it is in $\mathcal{I}_{S}^{+}$.

By Fact 3.7 , the ideals $[\omega]^{<\omega}$ and $\mathcal{I}_{S}$ are weakly selective so, by Proposition 3.8, they are prism-friendly. Thus, we need only to check that each of these ideals satisfies the condition (\#) from the definition of rich ideal.

It is well known that (\#) holds for $[\omega]^{<\omega}$. To check that (\#) also holds for $\mathcal{I}_{S}$, fix a countable basis $\mathcal{B}$ for the topology on $\mathbb{Q}$ and pick an $A \in \mathcal{I}_{S}^{+}$. By Fact 3.6, we can assume that $A \in \operatorname{Perf}(\mathbb{Q})$. Let $\left\{B_{n}: n<\omega\right\}$ be an 
enumeration of $\mathcal{B}_{A}=\{B \in \mathcal{B}:|B \cap A|=\omega\}$ and construct $\left\{a_{s}: s \in 2^{<\omega}\right\}$ by induction on the length of $s$ in such a way that $\left\{a_{s}: s \in 2^{n}\right\} \in\left[A \cap B_{n}\right]^{2^{n}}$ and that $\left\{a_{s}: s \in 2^{n}\right\} \cap \bigcup\left\{a_{t}: t \in 2^{<n}\right\}=\emptyset$ for every $n<\omega$. If for $x \in 2^{\omega}$ we put $A_{x}=\left\{a_{x \uparrow n}: n<\omega\right\}$, then $A_{x} \in \mathcal{I}_{S}^{+}$for every $x \in 2^{\omega}$, since $A_{x}$ is dense in $A$. Then $\mathcal{A}=\left\{A_{x}: x \in 2^{\omega}\right\}$ is almost disjoint and satisfies (\#).

Definition 3.9 Let $X$ be a countably infinite set and let $\mathcal{J} \subseteq \mathcal{P}(X)$ be an ideal on $X$ containing all singletons. The Fubini product of the ideals $[\omega]^{<\omega}$ and $\mathcal{J}$ is the ideal $\mathcal{K}$ on $\omega \times X$ denoted $[\omega]^{<\omega} \otimes \mathcal{J}$ and defined as the family of all subsets $A$ of $\omega \times X$ such that

$$
\operatorname{supp}(A) \stackrel{\text { def }}{=}\left\{n<\omega:(A)_{n} \in \mathcal{J}^{+}\right\} \text {is finite, }
$$

where $(A)_{n}=\{x \in X:\langle n, x\rangle \in A\}$.

Lemma 3.13 If $\mathcal{J}$ is a $Q$-like ideal, then $\mathcal{K}=[\omega]^{<\omega} \otimes \mathcal{J}$ is also Q-like.

Proof. Let $A \in \mathcal{K}^{+}$. For each $n \in \operatorname{supp}(A)$ let $\left\{A_{n}^{m} \in\left[(A)_{n}\right]^{\omega}: m<\omega\right\}$ be a family from the definition of $Q$-like for $(A)_{n} \in \mathcal{J}^{+}$. Then the family $\left\{\{n\} \times A_{n}^{m}: n \in \operatorname{supp}(A) \& m<\omega\right\}$ satisfies the definition of $Q$-like for the set $A$.

Lemma 3.14 Let $X$ be a countably infinite set, $\mathcal{J}$ a prism-friendly ideal on $X, P$ a prism in $2^{\omega \times X}, I \in[\omega]^{\omega}$, and let $\left\langle A_{n} \in \mathcal{J}^{+}: n \in I\right\rangle$ be arbitrary. Then, there are a subprism $Q$ of $P$, a set $J \in[I]^{\omega},\left\langle B_{n} \in \mathcal{P}\left(A_{n}\right) \cap \mathcal{J}^{+}: n \in J\right\rangle$, and an $i<2$, such that $g\lceil B$ is constant equal $i$ for every $g \in Q$ provided that $B=\bigcup\left\{\{n\} \times B_{n}: n \in J\right\}$. In particular, if $\mathcal{J}$ is prism-friendly, then so is $\mathcal{K}=[\omega]^{<\omega} \otimes \mathcal{J}$.

Proof. We can suppose that $I=\omega$. If $P$ is a singleton the lemma follows easily from the fact that $\mathcal{J}$ is an ideal containing the singletons and the pigeon hole principle. So, suppose that $P \in \operatorname{Perf}\left(2^{\omega \times X}\right)$. Let $f$ be a function witnessing that $P$ is a prism. By Remark 1.1 we can assume that $f$ is defined on $\mathfrak{C}^{\alpha}$ for some $0<\alpha<\omega_{1}$. We will construct a subprism $Q_{0}$ of $P$ and a sequence $\left\langle B_{n} \in\left[A_{n}\right]^{\omega} \cap \mathcal{J}^{+}: n<\omega\right\rangle$ such that for every $n<\omega$

$$
g\left\lceil\{n\} \times B_{n} \text { is constant for every } g \in Q_{0} .\right.
$$

This will be done using Proposition 1.3. 
For each $n<\omega$ let $\mathcal{D}_{n}$ be the collection of all pairwise disjoint families $\mathcal{E} \in\left[\mathbb{P}_{\alpha}\right]^{<\omega}$ such that there exists an $A_{\langle\mathcal{E}, n\rangle} \in\left[A_{n}\right]^{\omega} \cap \mathcal{I}^{+}$with the property that for every $E \in \mathcal{E}$

$$
f(h) \uparrow\{n\} \times A_{\langle\mathcal{E}, n\rangle}=f\left(h^{\prime}\right) \uparrow\{n\} \times A_{\langle\mathcal{E}, n\rangle} \text { for all } h, h^{\prime} \in E .
$$

Clearly, each $\mathcal{D}_{n}$ is closed under refinaments. To see that $\mathcal{D}_{n}$ satisfies the condition ( $\dagger$ ) from Proposition 1.3 pick $\mathcal{E} \in \mathcal{D}_{n}$ and $E \in \mathbb{P}_{\alpha}$ such that $E \cap \bigcup \mathcal{E}=\emptyset$. Decreasing $A_{\langle\mathcal{E}, n\rangle}$, if necessary, we can assume that $X \backslash A_{\langle\mathcal{E}, n\rangle}$ is infinite. Let $b_{n}: \omega \times X \rightarrow X$ be any bijection such that $b_{n}(n, a)=a$ for every $a \in A_{\langle\mathcal{E}, n\rangle}$. This bijection induces a homeomorphism $f_{n}: 2^{\omega \times X} \rightarrow 2^{X}$ defined by $f_{n}(g)(x)=g\left(b_{n}^{-1}(x)\right)$ for every $g \in 2^{\omega \times X}$ and $x \in X$. Clearly, $f_{n}$ is continuous and injective. Hence, $Q^{*}=\left(f_{n} \circ f\right)[E]$ is a prism in $2^{X}$. Since $\mathcal{J}$ is prism-friendly, we can find a subprism $Q^{* *}$ of $Q^{*}$, an $A^{\prime} \in\left[A_{\langle\mathcal{E}, n\rangle}\right]^{\omega} \cap \mathcal{J}^{+}$, and an $i<2$ such that $g\left[A^{\prime}\right]=\{i\}$ for every $g \in Q^{* *}$. But $Q^{* *}=f_{n}\left[E^{\prime}\right]$ for some $E^{\prime} \in \mathbb{P}_{\alpha} \cap \mathcal{P}(E)$. So, if we put $\mathcal{E}^{\prime}=\mathcal{E} \cup\left\{E^{\prime}\right\}$ and $A_{\left\langle\mathcal{E}^{\prime}, n\right\rangle}=A^{\prime}$ we get that $\mathcal{E}^{\prime} \in \mathcal{D}_{n}$ and the condition $(\dagger)$ is satisfied. Thus, by Proposition 1.3, for every $n<\omega$ there exists a family $\mathcal{E}_{n}=\left\{E_{k}: k<2^{n}\right\} \in \mathcal{D}_{n}$ of pairwise disjoint sets with $E^{0}=\bigcap_{n<\omega} \cup \mathcal{E}_{n} \in \mathbb{P}_{\alpha}$. We will prove that $Q_{0}=f\left[E^{0}\right]$ satisfies (3.1) with some sequence $\left\langle B_{n}: n<\omega\right\rangle$.

To see this fix an $n<\omega$, for each $k<2^{n}$ pick an $h_{k} \in E_{k}$, and define $\varphi_{n}: A_{\left\langle\mathcal{E}_{n}, n\right\rangle} \rightarrow 2^{2^{n}}$ by $\varphi_{n}(p)(k)=f\left(h_{k}\right)(n, p)$. Since $A_{\left\langle\mathcal{E}_{n}, n\right\rangle} \in \mathcal{I}^{+}$and $\mathcal{J}$ is an ideal, we can find an $s_{n} \in 2^{2^{n}}$ such that $B_{n}=\varphi_{n}^{-1}\left(s_{n}\right) \in \mathcal{J}^{+}$. To see that $B_{n}$ satisfies (3.1), pick a $g \in Q_{0}$. Then there exists a $k<2^{n}$ and an $h \in E_{k}$ such that $g=f(h)$. Since $B_{n} \subseteq A_{\left\langle\mathcal{E}_{n}, n\right\rangle}$, by (3.2) we have that $g\left\lceil\{n\} \times B_{n}=\right.$ $f\left(h_{k}\right)\left\lceil\{n\} \times B_{n}\right.$. In particular, $g(p)=f\left(h_{k}\right)(n, p)=\varphi_{n}(p)(k)=s_{n}(k)$ for every $p \in B_{n}$. So, $g\left\lceil\{n\} \times B_{n}\right.$ is constant equal to $s_{n}(k)$ and (3.1) holds.

To finish the proof of the lemma pick a $b_{n} \in B_{n}$ for each $n<\omega$. Then, the set $S=\left\{\left\langle n, b_{n}\right\rangle \in\{n\} \times B_{n}: n<\omega\right\}$ is a selector for $\left\{\{n\} \times B_{n}: n<\omega\right\}$. Let $\mathcal{I}=[\omega \times X]^{<\omega}$. Then $\mathcal{I}$ is weakly selective and $S \in \mathcal{I}^{+}$. If we identify $2^{\omega \times X}$ with $\mathcal{P}(\omega \times X)$, then $Q_{0}$ can be treated as a prism in $\mathcal{P}(\omega \times X)$. Since $[\omega \times X]^{\omega}$ is residual in $\mathcal{P}(\omega \times X)$, by Proposition 1.2 we can assume that $Q_{0}$ is a prism in $[\omega \times X]^{\omega}$. So, by Proposition 3.8, there exist a subprism $Q$ of $Q_{0}$, a set $S_{0} \in[S]^{\omega}$, and an $i<2$ such that $g\left[S_{0}\right]=\{i\}$ for every $g \in Q$. Define $J=\left\{n<\omega:\left\langle n, b_{n}\right\rangle \in S_{0}\right\}$.

To see that the conclusion of the lemma holds take a $g \in Q$ and an $\langle n, b\rangle \in B$. Then $n \in J$ and $b \in B_{n}$. So, by $(3.1), g(n, b)=g\left(n, b_{n}\right)=i$, since $\left\langle n, b_{n}\right\rangle \in S_{0}$. 
Lemma 3.15 Let $X$ be a countably infinite set and let $\mathcal{J} \subseteq \mathcal{P}(X)$ be an ideal containing all singletons and satisfying condition (\#) from the definition of a rich ideal. Then the ideal $\mathcal{K}=[\omega]^{<\omega} \otimes \mathcal{J}$ also satisfies (\#). In particular, if $\mathcal{J}$ is rich, then so is $\mathcal{K}$.

Proof. Let $A \in \mathcal{K}^{+}$. Then $\operatorname{supp}(A)$ is infinite. Let $\mathcal{A}=\left\{A_{\xi}: \xi<\mathfrak{c}\right\} \subseteq$ $[\operatorname{supp}(A)]^{\omega}$ be an almost disjoint family. Since $\mathcal{J}$ satisfies $(\#)$, for every $n<\omega$ there exists an almost disjoint family $\mathcal{B}_{n}=\left\{B_{\xi}^{n}: \xi<\mathfrak{c}\right\} \subseteq \mathcal{P}\left((A)_{n}\right) \cap \mathcal{J}^{+}$. If for every $\xi<\mathfrak{c}$ we define

$$
U_{\xi}=\bigcup\left\{\{n\} \times B_{\xi}^{n}: n \in A_{\xi}\right\},
$$

then the family $\left\{U_{\xi}: \xi<\mathfrak{c}\right\} \subseteq \mathcal{P}(A) \cap \mathcal{K}^{+}$works. The other part of the lemma is consequence of this and of Lemma 3.14 .

\subsection{An $\omega_{1}$-generated crowded bad point}

Definition 3.10 If $\mathcal{U}$ and $\mathcal{V}$ are ultrafilters, then the Fubini product of $\mathcal{U}$ and $\mathcal{V}$ is defined as

$$
\mathcal{U} \otimes \mathcal{V}=\left\{A \subseteq \omega \times \omega:\left\{n:(A)_{n} \in \mathcal{V}\right\} \in \mathcal{U}\right\} .
$$

Proposition 3.16 (Folklore) If $\mathcal{U}$ and $\mathcal{V}$ are non-principal ultrafilters in $\omega$ then $\mathcal{U} \otimes \mathcal{V}$ is a non-principal ultrafilter which is not a $P$-point, a $Q$-point, or even an $\omega_{1}-O K$ point.

Proof. It is easy to see that $\mathcal{U} \otimes \mathcal{V}$ is a non-principal ultrafilter. To see that $\mathcal{U} \otimes \mathcal{V}$ cannot be a $P$-point observe that the set $\left\{L_{m}: m<\omega\right\}$ of all sections $L_{m}=\{\langle m, n\rangle: n \in \omega\}$ is a partition of $\omega \times \omega$ into infinite pieces not in $\mathcal{U} \otimes \mathcal{V}$ and that every $X \in \mathcal{U} \otimes \mathcal{V}$ intersects infiniteley many $L_{m}$ 's on an infinite set.

To see that $\mathcal{U} \otimes \mathcal{V}$ cannot be a $Q$-point consider the partial partition $\left\{P_{n}: n<\omega\right\}$ of $\omega \times \omega$ where $P_{n}=\{\langle m, n\rangle: m \leq n\}$ for every $n<\omega$. Notice that $\bigcup_{n<\omega} P_{n} \in \mathcal{U} \otimes \mathcal{V}$. Let $\mathcal{P} \subseteq[\omega \times \omega]^{<\omega}$ be a partition of $\omega \times \omega$ such that $\left\{P_{n}: n<\omega\right\} \subseteq \mathcal{P}$. It is easy to see that there is no $X \in \mathcal{U} \otimes \mathcal{V}$ such that $|X \cap P| \leq 1$ for every $P \in \mathcal{P}$.

To see that $\mathcal{U} \otimes \mathcal{V}$ is not an $\omega_{1}$-OK point consider $\left\{V_{n}: n<\omega\right\} \subseteq \mathcal{U} \otimes \mathcal{V}$, where $V_{n}=\bigcup_{m>n} L_{m}$. By the way of contradiction, suppose that the sequence $\overline{\mathcal{U}}=\left\langle U_{\xi} \in \mathcal{U} \otimes \mathcal{V}: \xi<\omega_{1}\right\rangle$ is OK for $\left\{V_{n}: n<\omega\right\}$. Then, by the 
pigeon hole principle, there exist an $m<\omega$ and an $X \in\left[\omega_{1}\right]^{\omega_{1}}$ such that $\left(U_{\xi}\right)_{m} \in \mathcal{V}$ for every $\xi \in X$. Pick ordinals $\xi_{1}<\xi_{2}<\cdots<\xi_{m}$ in $X$. Since $\overline{\mathcal{U}}$ is OK for $\left\{V_{n}: n<\omega\right\}$ we have that $\bigcap_{i=1}^{m} U_{\xi_{i}} \subseteq^{*} V_{m} \subseteq \omega \times \omega \backslash L_{m}$. Therefore, $\left|\bigcap_{i=1}^{m} U_{\xi_{i}} \cap L_{m}\right|<\omega$. But also, $\left(\bigcap_{i=1}^{m} U_{\xi_{i}}\right)_{m}=\bigcap_{i=1}^{m}\left(U_{\xi_{i}}\right)_{m} \in \mathcal{V}$. This implies that $\left|\left(\bigcap_{i=1}^{m} U_{\xi_{i}}\right) \cap L_{m}\right|=\omega$, which is a contradiction.

Given $f, g \in \omega^{\omega}$ we write $g \leq^{*} f$ provided that $g(n) \leq f(n)$ for all but finitely many $n<\omega$. We say that an $F \subseteq \omega^{\omega}$ is dominating provided that for every $g \in \omega^{\omega}$ there exists an $f \in F$ such that $g \leq^{*} f$. The dominating number $\mathfrak{d}$ is defined as the minimum cardinality of a dominating family in $\omega^{\omega}$. This and other cardinal invariants have been studied extensively in the literature. See for example [4] or [1]. It is easy to show that $\omega_{1} \leq \mathfrak{d} \leq \mathfrak{c}$ and that this is all that can be said in ZFC about the value of $\mathfrak{d}$. For instance, the continuum hipothesis implies that $\mathfrak{d}=\omega_{1}=\mathfrak{c}$, while Martin's Axiom + $\mathfrak{c}>\omega_{1}$ imply that $\mathfrak{d}=\mathfrak{c}>\omega_{1}$. See, for example [24].

In [10, sec. 1.3] Ciesielski and Pawlikowski proved that a weak version of $\mathrm{CPA}_{\text {prism }}^{\text {game }}$, called $\mathrm{CPA}_{\text {cube }}$, implies that $\operatorname{cof}(\mathcal{N})=\omega_{1}<\mathfrak{c} .{ }^{5}$ It is known that this fact implies that $\mathfrak{d}=\omega_{1}$.

Also, it is not difficult to prove that $\mathfrak{d}=\omega_{1}$ implies that for every countable infinite set $X$ there is an $F \subseteq\left(\left[\omega_{1}\right]^{<\omega}\right)^{X}$ of cardinality $\omega_{1}$ which is $\subseteq$-dominant, that is, such that

for every $g \in\left(\left[\omega_{1}\right]^{<\omega}\right)$ there is an $f \in F$ with $g(x) \subseteq f(x)$ for all $x \in X$.

This follows from the fact that $\left(\left[\omega_{1}\right]^{<\omega}\right)^{X}=\bigcup_{\alpha<\omega_{1}}\left([\alpha]^{<\omega}\right)^{X}$. This is the form of $\mathfrak{d}=\omega_{1}$ which we will use in the next proposition.

Proposition 3.17 Assume $\mathfrak{d}=\omega_{1}$ and let $X$ and $Y$ be countably infinte sets. If $\mathcal{U}$ and $\mathcal{V}$ are $\omega_{1}$-generated ultrafilters on $X$ and $Y$, respectively, then their Fubini product $\mathcal{U} \otimes \mathcal{V}$ is also $\omega_{1}$-generated.

Proof. Let $\left\{U_{\alpha}: \alpha<\omega_{1}\right\}$ and $\left\{V_{\beta}: \beta<\omega_{1}\right\}$ be the bases for $\mathcal{U}$ and $\mathcal{V}$, respectively. Since $\mathfrak{d}=\omega_{1}$, there exists a $\subseteq$-dominant family $\left\langle f_{\gamma}: \gamma<\omega_{1}\right\rangle \subseteq$ $\left(\left[\omega_{1}\right]^{<\omega}\right)^{X}$. We claim that the family $\left\{W_{\alpha, \gamma}:\langle\alpha, \gamma\rangle \in \omega_{1} \times \omega_{1}\right\} \subseteq \mathcal{U} \otimes \mathcal{V}$, where $W_{\alpha, \gamma}=\bigcup\left\{\{x\} \times \bigcap_{\beta \in f_{\gamma}(x)} V_{\beta}: x \in U_{\alpha}\right\}$, is a basis for $\mathcal{U} \otimes \mathcal{V}$. To check this, pick an $A \in \mathcal{U} \otimes \mathcal{V}$. Then, $\{x \in X:\{y:\langle x, y\rangle \in A\} \in \mathcal{V}\} \in \mathcal{U}$. Pick an $\alpha<\omega_{1}$ such that $U_{\alpha} \subseteq\{x \in X:\{y:\langle x, y\rangle \in A\} \in \mathcal{V}\}$. Then, given an

\footnotetext{
${ }^{5} \operatorname{cof}(\mathcal{N})=\min \{|\mathcal{A}|: \mathcal{A} \subseteq \mathcal{N} \forall \mathrm{X} \in \mathcal{N} \exists \mathrm{Y} \in \mathcal{A}(\mathrm{X} \subseteq \mathrm{Y})\}$, where $\mathcal{N}$ is the null ideal on $\mathfrak{C}$.
} 
$x \in U_{\alpha}$ there exists a $\beta_{x}<\omega_{1}$ such that $V_{\beta_{x}} \subseteq\{y \in Y:\langle x, y\rangle \in A\}$. This implies that $\{x\} \times V_{\beta_{x}} \subseteq A$ for every $x \in U_{\alpha}$.

Consider the function $g: X \rightarrow\left[\omega_{1}\right]^{<\omega}$ defined as

$$
g(x)= \begin{cases}\left\{\beta_{x}\right\} & \text { if } x \in U_{\alpha} \\ \emptyset & \text { otherwise }\end{cases}
$$

Since $\left\langle f_{\gamma}: \gamma<\omega_{1}\right\rangle$ is a $\subseteq$-dominant family, there exists a $\gamma<\omega_{1}$ such that $g(x) \subseteq f_{\gamma}(x)$ for every $x \in U_{\alpha}$. This implies that $\beta_{x} \in f_{\gamma}(x)$ and that $\{x\} \times \bigcap_{\beta \in f_{\gamma}(x)} V_{\beta} \subseteq A$ for every $x \in U_{\alpha}$. Hence, $W_{\alpha, \gamma} \subseteq A$.

Theorem 3.18 $\mathrm{CPA}_{\text {prism }}^{\text {game }}$ implies that there exists an $\omega_{1}$-generated crowded ultrafilter which is not a $P$-point, a $Q$-point, or even an $\omega_{1}$-OK point.

PROOF. CPA prism implies the existence of an $\omega_{1}$-generated crowded ultrafilter $\mathcal{U}$ on $\mathbb{Q}$, see $[9$, prop. 4.25]. We will show that $\mathcal{U} \otimes \mathcal{U}$ is as desired.

By Proposition 3.16, it is not a $P$-point, a $Q$-point, or an $\omega_{1}$-OK point.

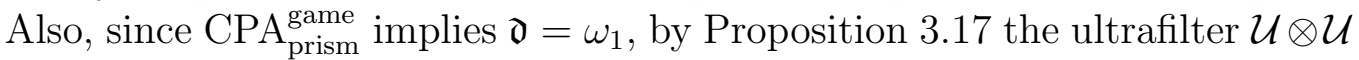
is $\omega_{1}$-generated by some family $\mathcal{B}$.

To see that $\mathcal{U} \otimes \mathcal{U}$ can be treated as crowded, consider $\mathbb{Q} \times \mathbb{Q}$ as the product of $\left\langle\mathbb{Q}, \tau_{d}\right\rangle$ and $\left\langle\mathbb{Q}, \tau_{s}\right\rangle$, where $\tau_{d}$ is the discrete topology and $\tau_{s}$ is the standard topology. Then $\mathbb{Q} \times \mathbb{Q}$ is homeomorphic to $\mathbb{Q}$.

For $B \in \mathcal{B}$ let $\left.\bar{B}=\left\{x:(B)_{x} \in B\right\} \in \mathcal{U}\right\}$. Using Fact 3.6, for every $x \in \bar{B}$ we can choose a subset $B^{x} \in \operatorname{Perf}(\mathbb{Q})$ of $(B)_{x}$. Let $B^{*}=\bigcup\left\{\{x\} \times B^{x}: x \in \bar{B}\right\}$. Then $B^{*}$ is a perfect subset of $\mathbb{Q} \times \mathbb{Q}$. Thus, $\left\{B^{*}: B \in \mathcal{B}\right\}$ is a basis of $\mathcal{U} \otimes \mathcal{U}$ of cardinality $\omega_{1}$ formed with perfect subsets of $\mathbb{Q} \times \mathbb{Q}$.

\subsection{A crowded $Q$-point which is not an $\omega_{1}-\mathrm{OK}$ point}

Definition 3.11 Let $X$ be a countably infinite set and let $\mathcal{J} \subseteq \mathcal{P}(X)$ be an ideal on $X$. If $A, B \in \mathcal{J}^{+}$we write $A \preceq \mathcal{J} B$ if and only if $A \backslash B \in \mathcal{J}$.

Definition 3.12 Let $X$ be a countably infinite set and let $\mathcal{J} \subseteq \mathcal{P}(X)$ be an ideal on $X$. We say that $\mathcal{J}$ has the extension property provided that for every $\preceq^{\mathcal{J}}$-decreasing sequence $\left\langle A_{n} \in \mathcal{J}^{+}: n<\omega\right\rangle$ there exists an $A \in \mathcal{J}^{+}$ such that $A \preceq \mathcal{J} A_{n}$ for every $n<\omega$. 
Let $X$ and $\mathcal{J}$ be as above and let $\mathcal{K}=[\omega]^{<\omega} \otimes \mathcal{J}$. We will consider a relation $\sqsubseteq$ defined on $\mathcal{K}^{+}$as

$A \sqsubseteq B \Leftrightarrow \operatorname{supp}(A) \subseteq^{*} \operatorname{supp}(B) \&(A)_{n} \preceq^{\mathcal{J}}(B)_{n} \quad \forall n \in \operatorname{supp}(A) \cap \operatorname{supp}(B)$.

Note that for $A, B \in \mathcal{K}^{+}$

$$
A \subseteq B \Longrightarrow A \sqsubseteq B \Longrightarrow A \preceq \preceq^{\mathcal{K}} B
$$

but none of these implications can be reversed. Also, it is not difficult to see that the relation $\sqsubseteq$ is not transitive. Nevertheless, we say that for $\xi<\omega_{1}$ a sequence $\left\langle U_{\eta} \in \mathcal{K}^{+}: \eta<\xi\right\rangle$ is $\sqsubseteq$-decreasing provided $U_{\eta} \sqsubseteq U_{\zeta}$ for every $\zeta<\eta<\xi$.

Lemma 3.19 Let $X$ be a countably infinite set, let $\mathcal{J} \subseteq \mathcal{P}(X)$ be an ideal on $X$ with the extension property, and let $\mathcal{K}=[\omega]^{<\omega} \otimes \mathcal{J}$. Then, for every $\xi<\omega_{1}$ and every $\sqsubseteq$-decreasing sequence $\left\langle U_{\eta} \in \mathcal{K}^{+}: \eta<\xi\right\rangle$ there exists a $C \in \mathcal{K}^{+}$ such that $C \sqsubseteq U_{\eta}$ for every $\eta<\xi$. Moreover, the sequence $\left\langle U_{\eta}: \eta \leq \xi\right\rangle$ is $\sqsubseteq$-decreasing for every $U_{\xi} \in \mathcal{P}(C) \cap \mathcal{K}^{+}$.

Proof. Let $\left\langle U_{\eta} \in \mathcal{K}^{+}: \eta<\xi\right\rangle$ be a $\sqsubseteq$-decreasing sequence. Since the sequence $\left\langle\operatorname{supp}\left(U_{\eta}\right): \eta<\xi\right\rangle$ is in particular $\subseteq^{*}$-decreasing, we can find an $S \in[\omega]^{\omega}$ such that $S \subseteq^{*} U_{\eta}$ for every $\eta<\xi$. For each $m \in S$ consider the set $I_{m}=\left\{\eta<\xi: m \in \operatorname{supp}\left(U_{\eta}\right)\right\}$. Then, since $\mathcal{J}$ has the extension property, we can find a $C_{m} \in \mathcal{J}^{+}$such that $C_{m} \preceq \mathcal{J}\left(U_{\eta}\right)_{m}$ for every $\eta \in I_{m}$. Put $C=\bigcup\left\{\{m\} \times C_{m}: m \in S\right\}$. Then clearly $C \sqsubseteq U_{\eta}$ for every $\eta<\xi$. The additional part follows from the fact that $U \subseteq C \sqsubseteq V$ implies $U \sqsubseteq V$.

Lemma 3.20 Let $X, \mathcal{J}$, and $\mathcal{K}$ be as above. Let $\left\langle U_{\xi} \in \mathcal{K}^{+}: \xi<\omega_{1}\right\rangle$ be a $\sqsubseteq$-decreasing sequence in $\mathcal{K}^{+}$such that for every $g \in \omega \times X$ there exists a $\xi<\omega_{1}$ such that $g \uparrow U_{\xi}$ is constant. Then, the family $\left\{U_{\xi}: \xi<\omega_{1}\right\}$ forms a base for a non-principal ultrafilter on $\omega \times X$ which is not an $\omega_{1}-O K$ point.

Proof. We check first that the family $\left\{U_{\xi}: \xi<\omega_{1}\right\}$ has SFIP. So, choose $\xi_{0}<\cdots<\xi_{n}<\omega_{1}$. Since $U_{\xi_{n}} \sqsubseteq \cdots \sqsubseteq U_{\xi_{1}} \sqsubseteq U_{\xi_{0}}$ we can pick an $m \in$ $\bigcap_{i \leq n} \operatorname{supp}\left(U_{\xi_{i}}\right)$. If $I_{m}=\left\{\xi<\omega_{1}: m \in \operatorname{supp}\left(A_{\xi}\right)\right\}$, then $\left\{\xi_{i}: i \leq n\right\} \subseteq I_{m}$. Therefore, $\left(U_{\xi_{n}}\right)_{m} \preceq^{\mathcal{J}} \ldots \preceq^{\mathcal{J}}\left(U_{\xi_{0}}\right)_{m}$. This implies that $\left(\bigcap_{i \leq n} U_{\xi_{i}}\right)_{m} \in \mathcal{J}^{+}$. In particular, $\left(\bigcap_{i \leq n} U_{\xi_{i}}\right)_{m}$ is infinite and so is $\bigcap_{i \leq n} U_{\xi_{i}}$. Let $\mathcal{U}$ be a filter generated $\left\{U_{\xi}: \xi<\omega_{1}\right\}$. 
To see that $\mathcal{U}$ is actually an ultrafilter, pick any $A \subseteq \omega \times X$. Then, there exists a $\xi<\omega_{1}$ and an $i<2$ such that $\chi_{A} \uparrow U_{\xi}$ is constant equal $i$. If $i=0$ then $U_{\xi} \subseteq(\omega \times X) \backslash A$ and $(\omega \times X) \backslash A \in \mathcal{U}$. If $i=1$ then $U_{\xi} \subseteq A$ and $A \in \mathcal{U}$. Therefore, $\mathcal{U}$ is an ultrafilter and $\left\{U_{\xi}: \xi<\omega_{1}\right\}$ is a base for $\mathcal{U}$. Observe that $\mathcal{U}$ is non-principal because each set in $\mathcal{U}$ contains an infinite set $U_{\xi}$.

To see that $\mathcal{U}$ is not an $\omega_{1}$-OK point consider a sequence $\left\langle V_{n} \in \mathcal{U}: n<\omega\right\rangle$, where $V_{n}=\bigcup_{i>n}(\{i\} \times X)$. Suppose that there exists a $\left\langle W_{\xi} \in \mathcal{U}: \xi<\omega_{1}\right\rangle$ which is OK for $\left\langle V_{n} \in \mathcal{U}: n<\omega\right\rangle$. Since $\left\{U_{\xi}: \xi<\omega_{1}\right\}$ is a basis for $\mathcal{U}$, for every for every $\xi<\omega_{1}$ there exists a $U_{\alpha_{\xi}} \subseteq W_{\xi}$. This implies that

$$
\left\langle U_{\alpha_{\xi}}: \xi<\omega_{1}\right\rangle \text { is OK for }\left\langle V_{n} \in \mathcal{U}: n<\omega\right\rangle \text {. }
$$

By the pigeon hole principle, there exist a $T \in\left[\omega_{1}\right]^{\omega_{1}}$ and an $m<\omega$ such that $m=\min \left(\operatorname{supp}\left(U_{\alpha_{\xi}}\right)\right)$ for every $\xi \in T$. Hence, $T \subseteq I_{m}$. Pick any ordinals $\alpha_{\xi_{0}}<\cdots<\alpha_{\xi_{m}}$ in $T$. Since $\left\langle U_{\alpha_{\xi}}: \xi<\omega_{1}\right\rangle$ is OK for $\left\langle V_{n} \in \mathcal{U}:<\omega\right\rangle$ we have that $\bigcap_{i \leq m} U_{\alpha_{\xi_{i}}} \subseteq^{*} V_{m}$. Hence, $\left|\left(\bigcap_{i \leq m} U_{\alpha_{\xi_{i}}}\right) \cap(\{m\} \times X)\right|<\omega$ by the definition of $V_{m}$. On the other hand, $\left\{\alpha_{\xi_{i}}: i \leq m\right\} \subseteq I_{m}$. Therefore, $\left(U_{\alpha_{\xi_{0}}}\right)_{m} \preceq^{\mathcal{J}} \ldots \preceq^{\mathcal{J}}\left(U_{\alpha_{\xi_{m}}}\right)_{m}$. This implies that $\left|\left(\bigcap_{i \leq m} U_{\alpha_{\xi_{i}}}\right)_{m}\right|=\omega$. So, $\left|\left(\bigcap_{i \leq m} U_{\alpha_{\xi_{i}}}\right) \cap(\{m\} \times X)\right|=\omega$. This contradiction indicates that $\mathcal{U}$ cannot be an $\omega_{1}$-OK point.

Let $X, \mathcal{J}$, and $\mathcal{K}$ be as before and let $\mathcal{D} \subseteq \mathcal{J}^{+}$be dense in the sense that for every $A \in \mathcal{J}^{+}$there exists a $D \in \mathcal{D}$ such that $D \subseteq A$. Then, the family $\mathcal{D}^{*} \subseteq \mathcal{K}^{+}$consisting of the sets of the form $\bigcup\left\{\{n\} \times D_{n}: n \in I\right\}$ is dense in $\mathcal{K}^{+}$, where $I \in[\omega]^{\omega}$ and $D_{n} \in \mathcal{D}$ for every $n \in I$. Recall also that $\mathcal{P}_{\omega \times X}$ is the space of all partitions of $\omega \times X$ into finite pieces, as defined in Section 3 .

Theorem 3.21 Let $X$ be a countably infinite set, let $\mathcal{J} \subseteq \mathcal{P}(X)$ be an ideal with the extension property, and let $\mathcal{D} \subseteq \mathcal{J}^{+}$be dense. If $\mathcal{J}$ is prism-friendly

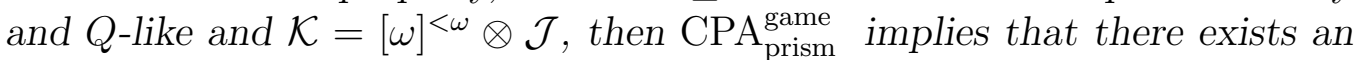
$\omega_{1}$-generated $Q$-point $\mathcal{U}$ on $\omega \times X$ which is not an $\omega_{1}$-OK point and such that $\mathcal{U} \cap \mathcal{D}^{*}$ is a basis for $\mathcal{U}$.

Proof. We construct a $\sqsubseteq$-decreasing sequence $\left\langle U_{\xi} \in \mathcal{K}^{+} \cap \mathcal{D}^{*}: \xi<\omega_{1}\right\rangle$ such that:

(i) For every $g \in 2^{\omega \times X}$ there exists a $\xi<\omega_{1}$ such that $g\left\lceil U_{\xi}\right.$ is constant.

(ii) For every $z \in \mathcal{P}_{\omega \times X}$ there exists a $\xi<\omega_{1}$ such that $\left|z(k) \cap U_{\xi}\right| \leq 1$ for every $k \in \omega$. 
If this construction is possible, then, by Lemma 3.19, $\left\{U_{\xi} \in \mathcal{D}^{*}: \xi<\omega_{1}\right\}$ is a basis for a non-principal ultrafilter $\mathcal{U}$ on $\omega \times X$ which is not an $\omega_{1}$-OK point. To see that $\mathcal{U}$ is a $Q$-point pick an arbitrary $z \in \mathcal{P}_{\omega \times X}$. Then, by condition (ii), there exists a $\xi<\omega_{1}$ such that $\left|z(k) \cap U_{\xi}\right| \leq 1$ for every $k<\omega$. Therefore, $\mathcal{U}$ is an $\omega_{1}$-generated $Q$-point.

Let $\mathcal{Y}=2^{\omega \times X} \cup \mathcal{P}_{\omega \times X}$ and consider it with the topology $\tau$ formed with all sets $A \subseteq \mathcal{Y}$ such that $A \cap 2^{\omega \times X}$ and $A \cap \mathcal{P}_{\omega \times X}$ are open in $2^{\omega \times X}$ and $\mathcal{P}_{\omega \times X}$, respectively. Then $\langle\mathcal{Y}, \tau\rangle$ is a Polish space. Note that, by Lemmas 3.13 and 3.14, the ideal $\mathcal{K}$ is $Q$-like and prism-friendly. For a prism $P$ in $\mathcal{Y}$ and $U \in \mathcal{K}^{+}$we choose a subprism $Q(U, P)$ of $P$ and $B(U, P) \in \mathcal{P}(U) \cap \mathcal{D}^{*}$ as follows.

- If $U \cap 2^{\omega \times X} \neq \emptyset$, then we can choose a subprism $P_{0} \subseteq 2^{\omega \times X}$ of $P$. The choice of $P_{0}$ is obvious if $P$ is a singleton; otherwise it follows from Proposition 1.2. Then $Q(U, P)$ is a subprism of $P_{0}$ such that $Q(U, P)$ and $B(U, P) \in \mathcal{P}(U) \cap \mathcal{K}^{+}$satisfy condition $(\bullet)$ from the definition of the prism-friendly ideal.

- If $U \cap 2^{\omega \times X}=\emptyset$, then $P$ is a prism in $\mathcal{P}_{\omega \times X}$. Then, by Lemma 3.11, there exist a subprism $Q(U, P)$ of $P$ and a $B(U, P) \in \mathcal{P}(U) \cap \mathcal{K}^{+}$such that $|z(k) \cap B(U, P)| \leq 1$ for every $z \in Q(U, P)$ and $k<\omega$.

We can also assume that $B(U, P) \in \mathcal{D}^{*}$, since $\mathcal{D}^{*}$ is dense in $\mathcal{K}^{+}$.

Also, for $\xi<\omega_{1}$ and a $\sqsubseteq$-decreasing sequence $\left\langle U_{\eta} \in \mathcal{K}^{+}: \eta<\xi\right\rangle$ let $C_{\xi}=$ $C\left(\left\langle U_{\eta}: \eta<\xi\right\rangle\right)$ be such that $C_{\xi} \sqsubseteq U_{\eta}$ for every $\eta<\xi$. Its existence follows from Lemma 3.19. Consider the following strategy $S$ for Player II:

$$
S\left(\left\langle\left\langle P_{\eta}, Q_{\eta}\right\rangle: \eta<\xi\right\rangle, P_{\xi}\right)=Q\left(C\left(\left\langle U_{\eta}: \eta<\xi\right\rangle\right), P_{\xi}\right),
$$

where the $U_{\eta}$ 's are defined inductively by $U_{\eta}=B\left(C\left(\left\langle U_{\zeta}: \zeta<\eta\right\rangle\right), P_{\eta}\right)$.

By $\mathrm{CPA}_{\text {prism }}^{\text {game }}$, the strategy $S$ is not a winning strategy for Player II. So, there exists a game $\left\langle\left\langle P_{\xi}, Q_{\xi}\right\rangle: \xi<\omega_{1}\right\rangle$ played according to $S$ in which Player II loses. Thus, $\mathcal{Y}=\bigcup_{\xi<\omega_{1}} Q_{\xi}$. Let $\left\langle U_{\xi} \in \mathcal{D}^{*}: \xi<\omega_{1}\right\rangle \subseteq \mathcal{K}^{+}$be the sequence created in this game. This sequence is $\sqsubseteq$-decreasing by construction and Lemma 3.19. By the observations made before we only need to check that $\left\langle U_{\xi}: \xi<\omega_{1}\right\rangle$ satisfy conditions (i) and (ii).

If $g \in 2^{\omega \times X}$, then there exists a $\xi<\omega_{1}$ such that $g \in Q_{\xi}$. So, $Q_{\xi} \subseteq 2^{\omega \times X}$ and, by the construction, $g \uparrow U_{\xi}$ is constant. This proves (i). Similarly, if $z \in \mathcal{P}_{\omega \times X}$, then there exists a $\xi<\omega_{1}$ such that $z \in Q_{\xi}$. Hence, $Q_{\xi} \subseteq \mathcal{P}_{\omega \times X}$ and, by the construction, $\left|z(k) \cap U_{\xi}\right| \leq 1$ for every $k<\omega$. This proves (ii). 
Corollary 3.22 $\mathrm{CPA}_{\text {prism }}^{\text {game }}$ implies that there exists an $\omega_{1}$-generated crowded $Q$-point which is not an $\omega_{1}-O K$ point

Proof. Consider $X=\omega \times \mathbb{Q}$ with the product topology, where $\omega$ has the discrete topology and $\mathbb{Q}$ has the subspace topology inherited from $\mathbb{R}$. Then $X$ is homeomorphic to $\mathbb{Q}$. We will find an ideal $\mathcal{J} \subseteq \mathcal{P}(X)$ to which we will apply Theorem 3.21.

Let $\mathcal{J}=[\omega]^{<\omega} \otimes \mathcal{I}_{S}$. It is clear that $\mathcal{J}$ contains all singletons. Also, $\mathcal{J}$ is prism-friendly by Lemmas 3.12 and 3.14 and $Q$-like by Lemmas 3.12 and 3.13. To see that $\mathcal{J}$ has the extension property pick a $\preceq \mathcal{J}$-decreasing sequence $\left\langle A_{n} \in \mathcal{J}^{+}: n<\omega\right\rangle$. By induction construct an increasing sequence $\left\langle n_{k}: k<\omega\right\rangle$ such that $n_{k} \in \operatorname{supp}\left(A_{k}\right) \backslash \operatorname{supp}\left(\bigcup_{i<k}\left(A_{k} \backslash A_{i}\right)\right)$. The choice can be made, since the set $\operatorname{supp}\left(\bigcup_{i<k}\left(A_{k} \backslash A_{i}\right)\right)$ is finite, as $\bigcup_{i<k}\left(A_{k} \backslash A_{i}\right) \in \mathcal{J}$. The choice of $n_{k}$ gives also $\left(\bigcup_{i<k}\left(A_{k} \backslash A_{i}\right)\right)_{n_{k}} \in \mathcal{I}_{S}$. Thus, $\left(\bigcap_{i \leq k} A_{i}\right)_{n_{k}} \notin \mathcal{I}_{S}$. Put $B=\bigcup\left\{\left\{n_{k}\right\} \times\left(\bigcap_{i \leq k} A_{i}\right)_{n_{k}}: k<\omega\right\}$. Then $B \in \mathcal{J}^{+}$and $B \preceq \mathcal{J} A_{n}$ for every $n<\omega$.

Since $\overline{\mathcal{D}}=\operatorname{Perf}(\mathbb{Q})$ is dense in $\left(\mathcal{I}_{S}\right)^{+}$, the family $\mathcal{D}=\overline{\mathcal{D}}^{*}$ is dense in $\mathcal{J}^{+}$. Applying Theorem 3.21 to $\mathcal{J}$ and $\mathcal{D}$, we can find an $\omega_{1}$-generated $Q$-point $\mathcal{U}$ on $\omega \times X$ which is not an $\omega_{1}$-OK point and such that $\mathcal{U} \cap \mathcal{D}^{*}$ contains a basis for $\mathcal{U}$. Since $\omega \times X$ is homeomorphic to $\mathbb{Q}$ and $\mathcal{D}^{*}$ consists of perfect set in $\omega \times X$, it follows that $\mathcal{U}$ is crowded.

\subsection{Crowded $\omega_{1}$-generated $\omega_{1}$-OK points}

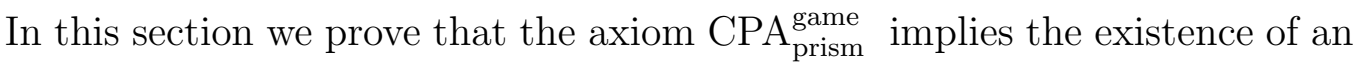
$\omega_{1}$-OK point which is not a $P$-point. For this, we follow the schema used in [23] for the construction of such an ultrafilter in the model of ZFC obtained by adding Sacks reals side-by-side. Since that proof uses $\mathrm{CH}$ in the ground model, we have to modify things a bit to make it work in the context of $\mathrm{CPA}_{\text {prism }}^{\text {game }}$. One possiblity for avoiding the use of $\mathrm{CH}$ is to replace it with some weaker principle consistent with $\mathrm{CPA}_{\text {prism }}^{\text {game }}$ like, for instance, $\mathfrak{d}=\omega_{1}$. Let $\Gamma$ denote the set of all nonzero limit ordinals below $\omega_{1}$. The following fact is a simple generalization of the remark above Proposition 3.17.

Fact $3.23\left(\mathfrak{d}=\omega_{1}\right)$ There exist a sequence $\left\langle g_{\delta}: \delta<\omega_{1}\right\rangle$ of functions from $\omega$ into $\left[\omega_{1}\right]^{<\omega}$ and a partition $\left\{S_{\delta} \in\left[\omega_{1}\right]^{\omega_{1}}: \delta<\omega_{1}\right\}$ of $\Gamma$ such that: 
- For every $h: \omega \rightarrow \omega_{1}$ there is a $\delta<\omega_{1}$ such that $h(n) \in g_{\delta}(n)$ for every $n<\omega$.

- $\bigcup \operatorname{rang}\left(g_{\delta}\right)=\min \left(S_{\delta}\right)$ for every $\delta<\omega_{1}$.

Fix a countably infinite set $X$ and put $\mathcal{P}=\{\{m\} \times X: m<\omega\}$. Then, $\mathcal{P}$ is a partition of $\omega \times X$ into infinitely many infinite pieces. The idea of the proof is to find a sequence $\left\langle U_{\alpha}: \alpha<\omega_{1}\right\rangle$ that forms a base for a nonprincipal ultrafilter $\mathcal{U}$ on $\omega \times X$ such that every $U_{\alpha}$ has infinite intersection with infinitely many members of $\mathcal{P}$ and, for each $\delta<\omega_{1}$,

$$
\left\langle U_{\alpha}: \alpha \in S_{\delta}\right\rangle \text { is OK for }\left\{\bigcap_{\eta \in g_{\delta}(n)} U_{\eta}: n<\omega\right\} \text {. }
$$

To see that such an $\mathcal{U}$ is an $\omega_{1}$-OK point pick $\left\langle V_{n}: n<\omega\right\rangle \in(\mathcal{U})^{\omega}$. Since the sequence $\left\langle U_{\alpha}: \alpha<\omega_{1}\right\rangle$ is a basis for $\mathcal{U}$, for every $n<\omega$ there is a $\xi_{n}<\omega_{1}$ such that $U_{\xi_{n}} \subseteq V_{n}$. Therefore, there exists a $\delta<\omega_{1}$ such that $\xi_{n} \in g_{\delta}(n)$ for every $n<\omega$. Then $\left\langle U_{\alpha}: \alpha \in S_{\delta}\right\rangle$ is OK for $\left\langle V_{n}: n<\omega\right\rangle$ since for any sequence $\alpha_{0}<\cdots<\alpha_{n}$ of elements in $S_{\delta}$ we have:

$$
\bigcap_{i \leq n} U_{\alpha_{i}} \subseteq^{*} \bigcap_{\eta \in g_{\delta}(n)} U_{\eta} \subseteq U_{\xi_{n}} \subseteq V_{n}
$$

Observe that $\mathcal{U}$ cannot be a $P$-point because each $U_{\alpha}$ intersects infinitely many members of $\mathcal{P}$ on an infinite set.

Let us start with fixing a rich ideal $\mathcal{J} \subseteq \mathcal{P}(X)$ and a dense $\mathcal{D} \subseteq \mathcal{J}^{+}$. We will consider the ideal $\mathcal{K}=[\omega]^{<\omega} \otimes \mathcal{J}$ on $\omega \times X$ and the set $\mathcal{D}^{*} \subseteq \mathcal{K}^{+}$as defined in Section 3.5. We also fix, for each $\xi \in \Gamma$, an enumeration $\left\{\xi_{i}: i<\omega\right\}$ of $\xi$.

Let $\mathcal{T}$ be the set of triples $\langle I, f, B\rangle$ satisfying the following requirements:

- $I$ is an infinite subset of $\omega$,

- $f \in \prod_{m<\omega}(\mathcal{P}(X) \cap \mathcal{D})$, and

- $B \in \prod_{m \in \omega}(\mathcal{P}(f(m)) \cap \mathcal{D})^{\omega_{1}}$ such that every $B(m)$ is a sequence of almost disjoint sets. 
If $\xi \leq \omega_{1}$ and $\left\langle\left\langle I_{\eta}, f_{\eta}, B_{\eta}\right\rangle \in \mathcal{T}: \eta<\xi\right\rangle$, the sequence $\left\langle U_{\eta}: \eta<\xi\right\rangle$ associated with it is defined by

$$
U_{\eta}=\bigcup\left\{\{m\} \times f_{\eta}(m): m \in I_{\eta}\right\} .
$$

Note that each $U_{\eta}$ is in $\mathcal{D}^{*}$.

To prove that the resulting ultrafilter $\mathcal{U}$ in our construction is in fact an $\omega_{1}$-OK point we will consider for every $\delta<\omega_{1}, \eta<\xi$, and $m<\omega$ the sets $K(\eta, m)=\left\{\zeta<\eta: f_{\eta}(m) \subseteq f_{\zeta}(m)\right\}$, the numbers $k_{\delta}(\eta, m)=\left|K(\eta, m) \cap S_{\delta}\right|$, and the functions $l_{\delta}$ defined by:

$$
l_{\delta}(\eta, m)=\left\{\begin{array}{lc}
\infty & \text { if } \bigcup \operatorname{rang}\left(g_{\delta}\right) \subseteq K(\eta, m) \\
-1 & \text { if } g_{\delta}(0) \nsubseteq K(\eta, m) \\
\max \left\{l<\omega: \bigcup g_{\delta}[l+1] \subseteq K(\eta, m)\right\} & \text { otherwise. }
\end{array}\right.
$$

Definition 3.13 For $\xi \leq \omega_{1}$ a sequence $\left\langle\left\langle I_{\eta}, f_{\eta}, B_{\eta}\right\rangle \in \mathcal{T}: \eta<\xi\right\rangle$ is $\operatorname{good}$ if:

(a) For every $\zeta<\eta<\xi$ and $m<\omega$, either $f_{\zeta}(m) \cap f_{\eta}(m)$ is finite, or there exists a $\gamma \leq \eta$ such that $f_{\eta}(m) \subseteq B_{\zeta}(m)(\gamma) \subseteq f_{\zeta}(m)$.

(b) For every $0<\eta<\xi$ and $m<\omega$ there exists a $\zeta<\eta$ such that $f_{\eta}(m) \subseteq B_{\zeta}(m)(\eta)$

(c) If $\eta<\xi$ is limit and $\left\{m_{i}: i<\omega\right\}$ is the increasing enumeration of $I_{\eta}$, then

$$
m_{i} \in \bigcap_{j \leq i} I_{\eta_{j}} \quad \text { and } \quad f_{\eta}\left(m_{i}\right) \subseteq \bigcap_{j \leq i} f_{\eta_{j}}\left(m_{i}\right),
$$

where $\left\{\eta_{j}: j<\omega\right\}$ is our fixed enumeration of $\eta$.

(d) $f_{\eta}(m)=B_{0}(m)(\eta)$ for every $m \in \omega \backslash I_{\eta}$.

(e) If $\eta+1<\xi$, then $I_{\eta+1} \subseteq I_{\eta}$ and $f_{\eta+1}(m) \subseteq B_{\eta}(m)(\eta+1) \subseteq f_{\eta}(m)$ for every $m \in I_{\eta+1}$.

(f) If $\delta<\omega_{1}, \eta<\xi$, and $\eta \in S_{\delta}$, then $l_{\delta}(\eta, m)>k_{\delta}(\eta, m)$ for every $m \in I_{\eta}$.

(g) If $\delta<\omega_{1}$,

$\eta<\xi$, and $\bigcup \operatorname{rang}\left(g_{\delta}\right) \subseteq \eta$, then

$$
\lim _{\substack{m \in I_{\eta} \\ m \rightarrow \infty}}\left(l_{\delta}(\eta, m)-k_{\delta}(\eta, m)\right)=\infty .
$$


Remark 3.24 It follows from (c) and (e) that if $\zeta<\eta<\xi$, then $I_{\eta} \subseteq^{*} I_{\zeta}$.

Remark 3.25 It is also easy to check that if $\xi \leq \omega_{1}$ is a limit ordinal, then the sequence $\left\langle\left\langle I_{\zeta}, f_{\zeta}, B_{\zeta}\right\rangle \in \mathcal{T}: \zeta<\xi\right\rangle$ is good if and only if the sequence $\left\langle\left\langle I_{\zeta}, f_{\zeta}, B_{\zeta}\right\rangle \in \mathcal{T}: \zeta<\eta\right\rangle$ is good for every $\eta<\xi$.

Remark 3.26 It is not difficult to see that

if $\alpha<\beta<\xi$, then $f_{\beta}(m) \subseteq f_{\alpha}(m)$ for all but finitely many $m \in I_{\beta}$.

If $\beta \in \Gamma$ this follows from (c). If $\Gamma \cap(\alpha, \beta]=\emptyset$, then it follows from (e). If $\Gamma \cap(\alpha, \beta] \neq \emptyset$, then there exist a maximal $\gamma \in \Gamma \cap(\alpha, \beta]$ and, by the above two cases, $f_{\beta}(m) \subseteq f_{\gamma}(m) \subseteq f_{\alpha}(m)$ for all but finitely many $m \in I_{\beta}$.

Remark 3.27 For every $0<\eta<\xi$ and $m<\omega$ there exists a $\gamma \leq \eta$ such that $f_{\eta}(m) \subseteq B_{0}(m)(\gamma)$. This follows from condition (b), since every strictly decreasing sequence of ordinals is finite.

The dual filter of an ideal $\mathcal{K}$ on a set $\omega \times X$ is the family $\mathcal{F}_{\mathcal{K}}$ defined as $\mathcal{F}_{\mathcal{K}}=\{(\omega \times X) \backslash A: A \in \mathcal{K}\}$. The importance of the definition of a good sequence derives from the following lemma.

Lemma 3.28 Let $X$ be a countably infinite set, $\mathcal{J} \subseteq \mathcal{P}(X)$ a rich ideal in $X$, $\mathcal{D} \subseteq \mathcal{J}^{+}$a dense family, and let $\mathcal{K}=[\omega]<\omega \otimes \mathcal{J}$. If $\left\langle\left\langle I_{\xi}, f_{\xi}, B_{\xi}\right\rangle \in \mathcal{T}: \xi<\omega_{1}\right\rangle$ is a good sequence such that for every $g \in 2^{\omega \times X}$ there exists a $\xi<\omega_{1}$ such that $g \uparrow U_{\xi}$ is constant, then $\left\langle U_{\xi} \in \mathcal{D}^{*}: \xi<\omega_{1}\right\rangle$ forms a base for a nonprincipal ultrafilter on $\omega \times X$ extending $\mathcal{F}_{\mathcal{K}}$ which is an $\omega_{1}$-OK point but not a P-point.

Proof. The fact that $\left\{U_{\xi}: \xi<\omega_{1}\right\} \subseteq \mathcal{D}^{*}$ follows immediately from the definition of $U_{\eta}$ and $\mathcal{D}^{*}$.

Next we prove that $\left\{U_{\xi}: \xi<\omega_{1}\right\}$ forms a base for a non-principal ultrafilter $\mathcal{U}$ on $\omega \times X$ extending the filter $\mathcal{F}_{\mathcal{K}}$. Given $\xi_{0}<\cdots<\xi_{n}$ pick a $\gamma \in \Gamma$ with $\gamma>\xi_{n}$. By (c), we have that almost every $m \in I_{\gamma}$ is in $\bigcap_{i<n} I_{\xi_{i}}$ and that $f_{\gamma}(m) \subseteq \bigcap_{i<n} f_{\xi_{i}}(m)$. Therefore, $\bigcap_{i<n} U_{\xi_{i}} \in \mathcal{K}^{+}$and $\left\{U_{\xi}: \xi<\omega_{1}\right\}$ can be extended to a proper filter $\mathcal{U}$ on $\omega \times X$. If $A \subseteq \omega \times X$, then $\chi_{A} \in 2^{\omega \times X}$ and there exist a $\xi<\omega_{1}$ and an $i<2$ such that $\chi_{A}\left\lceil U_{\xi}\right.$ is constant equal $i$. If $i=1$ then $U_{\xi} \subseteq A$ and $A \in \mathcal{U}$. If $i=0$ then $U_{\xi} \subseteq(\omega \times X) \backslash A$ so $(\omega \times X) \backslash A \in \mathcal{U}$. This proves that $\mathcal{U}$ is an ultrafilter and that $\left\{U_{\xi}: \xi<\omega_{1}\right\}$ 
is a base for $\mathcal{U}$. Since no $A \in \mathcal{K}$ contains any $U_{\xi}$, it follows that $\mathcal{U}$ extends $\mathcal{F}_{\mathcal{K}}$. In particular, $\mathcal{U}$ is non-principal.

To see that $\mathcal{U}$ is not a $P$-point notice that every $U_{\xi}$ intersects infinitely many pieces of the partition $\mathcal{P}=\{\{m\} \times X: m<\omega\}$ on an infinite set and so does every $V \in \mathcal{U}$.

To prove that $\mathcal{U}$ is an $\omega_{1}$-OK point it is enough to prove that for every $\delta<\omega_{1}$

$$
\left\langle U_{\alpha}: \alpha \in S_{\delta}\right\rangle \text { is OK for }\left\{\bigcap_{\eta \in g_{\delta}(n)} U_{\eta}: n<\omega\right\} \text {. }
$$

Pick $\delta<\omega_{1}$ and $\xi_{0}<\cdots<\xi_{n}$ in $S_{\delta}$. First, we prove that for every $m \in I_{\xi_{n}}$

$$
\text { either } \bigcap_{i \leq n} f_{\xi_{i}}(m) \text { is finite, or } \bigcap_{i \leq n} f_{\xi_{i}}(m) \subseteq \bigcap_{\eta \in g_{\delta}(n)} f_{\eta}(m)
$$

Indeed, assume that $\bigcap_{i \leq n} f_{\xi_{i}}(m)$ is infinite. Then, by part (a) of Definition 3.13, $\xi_{i} \in K\left(\xi_{n}, m\right) \cap S_{\delta}$ for each $i \leq n-1$. Therefore, $k_{\delta}\left(\xi_{n}, m\right) \geq n$ and $l_{\delta}\left(\xi_{n}, m\right) \geq n+1$ by Definition 3.13(f). In particular, $g_{\delta}(n) \subseteq K\left(\xi_{n}, m\right)$. Hence, by the definition of $K(\eta, m)$,

$$
\bigcap_{i \leq n} f_{\xi_{i}}(m) \subseteq f_{\xi_{n}}(m) \subseteq \bigcap_{\eta \in K\left(\xi_{n}, m\right)} f_{\eta}(m) \subseteq \bigcap_{\eta \in g_{\delta}(n)} f_{\eta}(m) .
$$

Also, Definition 3.13(c) implies that $f_{\xi_{n}}(m) \subseteq \bigcap_{\eta \in g_{\delta}(n)} f_{\eta}(m)$ for all but finitely many $m \in I_{\xi_{n}}$. Thus, the set $s=\left\{m \in I_{\xi_{n}}: \bigcap_{i \leq n} f_{\xi_{i}}(m) \not \subseteq \bigcap_{\eta \in g_{\delta}(n)} f_{\eta}(m)\right\}$ 
is finite. Moreover, by $(3.5), \bigcap_{i \leq n} f_{\xi_{i}}(m)$ is finite for every $m \in s$. So,

$$
\begin{aligned}
\bigcap_{i \leq n} U_{\xi_{i}} & =\bigcup_{m \in \bigcap_{i \leq n} I_{\xi_{i}}}\left(\{m\} \times \bigcap_{i \leq n} f_{\xi_{i}}(m)\right) \\
& \subseteq \bigcup_{m \in I_{\xi_{n}}}\left(\{m\} \times \bigcap_{i \leq n} f_{\xi_{i}}(m)\right) \\
& =\bigcup_{m \in s}\left(\{m\} \times \bigcap_{i \leq n} f_{\xi_{i}}(m)\right) \cup \bigcup_{m \in I_{\xi_{n} \backslash s}}\left(\{m\} \times \bigcap_{i \leq n} f_{\xi_{i}}(m)\right) \\
& \subseteq \bigcup_{m \in I_{\xi_{n}} \backslash s}\left(\{m\} \times \bigcap_{\eta \in g_{\delta}(n)} f_{\xi_{i}}(m)\right) \\
& \subseteq \bigcup_{m \in I_{\xi_{n}}}\left(\{m\} \times \bigcap_{\eta \in g_{\delta}(n)} f_{\xi_{i}}(m)\right)=\bigcup_{\eta \in g_{\delta}(n)} U_{\eta},
\end{aligned}
$$

which proves (3.4). So $\mathcal{U}$ is an $\omega_{1}$-OK point.

Lemma 3.29 Let $\left\langle\left\langle I_{\eta}, f_{\eta}, B_{\eta}\right\rangle \in \mathcal{T}: \eta<\xi\right\rangle$ be a sequence satisfying condition (a) from Definition 3.13 and let $\alpha<\beta<\xi$ and $m<\omega$ be such that $f_{\beta}(m) \subseteq B_{\alpha}(m)(\beta)$. Then $K(\beta, m)=K(\alpha, m) \cup\{\alpha\}$. In particular, if the sequence satisfies conditions (a) and (b) from Definition 3.13, then the set $K(\eta, m)$ is finite for every $\eta<\xi$ and $m<\omega$.

Proof. If $\eta \in K(\beta, m)$, then $\eta<\beta$ and $f_{\beta}(m) \subseteq f_{\eta}(m)$. Also, since $f_{\beta}(m) \subseteq B_{\alpha}(m)(\beta) \subseteq f_{\alpha}(m)$ we have that $\left|f_{\eta}(m) \cap f_{\alpha}(m)\right|=\omega$. If $\alpha<\eta$, then, by condition (a), there exists a $\gamma \leq \eta$ such that $f_{\eta}(m) \subseteq B_{\alpha}(m)(\gamma)$; therefore $f_{\eta}(m) \subseteq B_{\alpha}(m)(\gamma) \cap B_{\alpha}(m)(\beta)$, which is impossible. Thus, $\eta \leq \alpha$. If $\eta<\alpha$, then, again by (a), there is a $\gamma \leq \alpha$ such that $f_{\alpha}(m) \subseteq B_{\eta}(m)(\gamma)$. Since $B_{\eta}(m)(\gamma) \subseteq f_{\eta}(m)$, we conclude that $f_{\alpha}(m) \subseteq f_{\eta}(m)$. Therefore, $\eta \in K(\alpha, m)$ and this proves that $K(\beta, m) \subseteq K(\alpha, m) \cup\{\alpha\}$.

Since $f_{\beta}(m) \subseteq B_{\alpha}(m)(\beta) \subseteq f_{\alpha}(m)$ we have that $\alpha \in K(\beta, m)$. If $\eta \in$ $K(\alpha, m)$, then $f_{\alpha}(m) \subseteq f_{\eta}(m)$. But since $f_{\beta}(m) \subseteq B_{\alpha}(m)(\beta) \subseteq f_{\alpha}(m)$ we have that $f_{\beta}(m) \subseteq f_{\eta}(m)$. Therefore, $\eta \in K(\beta, m)$ and this proves that $K(\alpha, m) \cup\{\alpha\} \subseteq K(\beta, m)$. Thus, $K(\beta, m)=K(\alpha, m) \cup\{\alpha\}$. 
Since condition (b) implies that for every $0<\eta<\xi$ and $m<\omega$ there exists a $\zeta<\eta$ such that $f_{\eta}(m) \subseteq B_{\zeta}(m)(\eta)$, we have that for every $0<\eta<\xi$ there exists a $\zeta<\eta$ such that $K(\eta, m)=K(\zeta, m) \cup\{\zeta\}$. Since $K(0, m)=\emptyset$ for every $m<\omega$, we can prove, by induction on $\eta$, that $K(\eta, m)$ is finite for every $\eta<\xi$ and $m<\omega$.

Lemma 3.30 If $\xi \in \Gamma$ and $\left\langle\left\langle I_{\eta}, f_{\eta}, B_{\eta}\right\rangle \in \mathcal{T}: \eta<\xi\right\rangle$ is good, then there exists an $\left\langle I_{\xi}, f_{\xi}, B_{\xi}\right\rangle \in \mathcal{T}$ such that the sequence $\left\langle\left\langle I_{\eta}, f_{\eta}, B_{\eta}\right\rangle \in \mathcal{T}: \eta \leq \xi\right\rangle$ is good.

Proof. Let $\left\{\xi_{j}: j<\omega\right\}$ be the fixed enumeration of $\xi$. Since $S_{\delta}$ 's are pairwise disjoint, the set $\left\{\delta<\omega_{1}: \min \left(S_{\delta}\right)<\xi\right\}$ is countable and it can be enumerated as $\left\{\delta_{i}: i<\omega\right\}$. Let $\delta^{*}<\omega_{1}$ be such that $\xi \in S_{\delta^{*}}$. We define $I_{\xi}=\left\{m_{i}: i<\omega\right\}$ inductively. Suppose that $m_{j}$ has already been defined for every $j<i$. Put

$$
\varepsilon_{i}=\max \left(g_{\delta^{*}}(0) \cup g_{\delta^{*}}(0) \cup\left\{\xi_{j}: j \leq i\right\} \cup\left\{\min \left(S_{\delta_{j}}\right): j \leq i\right\}\right)+1<\xi .
$$

Note that $\varepsilon_{i}<\xi$ since Remark 3.24 implies that $I_{\varepsilon_{i}} \subseteq^{*} \bigcap_{j \leq i} I_{\xi_{j}}$. Thus, we can pick an $m_{i} \in I_{\varepsilon_{i}} \cap \bigcap_{j \leq i} I_{\xi_{j}}$ so that:

(i) $m_{i}>m_{j}$ for every $j<i$,

(ii) $l_{\delta_{j}}\left(\varepsilon_{i}, m_{i}\right)-k_{\delta_{j}}\left(\varepsilon_{i}, m_{i}\right)>i$ for every $j \leq i$, and

(iii) $f_{\varepsilon_{i}}\left(m_{i}\right) \subseteq \bigcap_{j \leq i} f_{\xi_{j}}\left(m_{i}\right) \cap \bigcap\left\{f_{\eta}\left(m_{i}\right): \eta \in g_{\delta^{*}}(0) \cup g_{\delta^{*}}(1)\right\}$.

Condition (ii) can be achieved since $\bigcup \operatorname{rang}\left(g_{\delta_{j}}\right) \subseteq \min \left(S_{\delta_{j}}\right)<\varepsilon_{i}<\xi$ and $\left\{\left\langle I_{\eta}, f_{\eta}, B_{\eta}\right\rangle: \eta<\xi\right\}$ is good, so, by (g),

$$
\lim _{\substack{m \in I_{\varepsilon_{i}} \\ m \rightarrow \infty}}\left(l_{\delta_{j}}\left(\varepsilon_{i}, m\right)-k_{\delta_{j}}\left(\varepsilon_{i}, m\right)\right)=\infty
$$

for every $j \leq i$. Condition (iii) can be ensured by Remark 3.26, since $\xi_{j}<\varepsilon_{i}$ for $j \leq i$ and $\eta<\varepsilon_{i}$ for all $\eta \in g_{\delta^{*}}(0) \cup g_{\delta^{*}}(1)$. This completes the inductive definition of $I_{\xi}$. Define $f_{\xi}: \omega \rightarrow \mathcal{D}$ as

$$
f_{\xi}(m)= \begin{cases}B_{\varepsilon_{i}}\left(m_{i}\right)(\xi) & \text { if } m=m_{i} \in I_{\xi} \\ B_{0}(m)(\xi) & \text { otherwise. }\end{cases}
$$


The $B_{\xi}$ can be defined by taking for each $m<\omega$ an arbitrary $\omega_{1}$-sequence of almost disjoint sets in $\mathcal{P}\left(f_{\xi}(m)\right) \cap \mathcal{D}$. This completes the definition of $\left\langle I_{\xi}, f_{\xi}, B_{\xi}\right\rangle$.

To make sure that (a) holds it is enough to check it only for the pair $\langle\eta, \xi\rangle$ in place of $\langle\zeta, \eta\rangle$. So, choose an $\eta<\xi$ and $m<\omega$. We need to show that either $f_{\xi}(m) \cap f_{\eta}(m)$ is finite, or there exists a $\gamma \leq \xi$ such that $f_{\xi}(m) \subseteq B_{\eta}(m)(\gamma)$. We will consider several cases.

$m \notin I_{\xi}$ : We will consider here two subcases.

$\eta=0$ : Then $f_{\xi}(m)=B_{0}(m)(\xi)=B_{\eta}(m)(\gamma)$ for $\gamma=\xi$.

$\eta>0$ : Apply Remark 3.27 to find $\gamma \leq \eta$ such that $f_{\eta}(m) \subseteq B_{0}(m)(\gamma)$. Since $f_{\xi}(m)=B_{0}(m)(\xi)$, we have that $\left|f_{\xi}(m) \cap f_{\eta}(m)\right|<\omega$.

$m=m_{i} \in I_{\xi}$ : We will consider here three subcases.

$\varepsilon_{i}<\eta$ : We will show that $\left|f_{\xi}(m) \cap f_{\eta}(m)\right|<\omega$. So, by way of contradiction, assume that $\left|f_{\xi}(m) \cap f_{\eta}(m)\right|=\omega$. Therefore, the sets $f_{\xi}(m) \cap f_{\eta}(m)=B_{\varepsilon_{i}}(m)(\xi) \cap f_{\eta}(m) \subseteq f_{\varepsilon_{i}}(m) \cap f_{\eta}(m)$ are infinite. So, by (a), there exists a $\gamma \leq \eta$ such that $f_{\eta}(m) \subseteq B_{\varepsilon_{i}}(m)(\gamma)$. Thus, $B_{\varepsilon_{i}}(m)(\xi) \cap B_{\varepsilon_{i}}(m)(\gamma)=f_{\xi}(m) \cap B_{\varepsilon_{i}}(m)(\gamma) \supseteq f_{\xi}(m) \cap f_{\eta}(m)$ is infinite, which is impossible, as $\gamma \leq \eta<\xi$. This implies that $\left|f_{\xi}(m) \cap f_{\eta}(m)\right|<\omega$.

$\varepsilon_{i}>\eta$ : If $f_{\varepsilon_{i}}(m) \cap f_{\eta}(m)$ is finite then so is $f_{\xi}(m) \cap f_{\eta}(m) \subseteq f_{\varepsilon_{i}}(m) \cap f_{\eta}(m)$. Otherwise, by (a), there is a $\gamma \leq \varepsilon_{i}$ such that $f_{\varepsilon_{i}}(m) \subseteq B_{\eta}(m)(\gamma)$. So, $f_{\xi}(m)=B_{\varepsilon_{i}}(m)(\xi) \subseteq f_{\varepsilon_{i}}(m) \subseteq B_{\eta}(m)(\gamma)$.

$\varepsilon_{i}=\eta$ : Clearly $f_{\xi}(m)=B_{\varepsilon_{i}}(m)(\xi)=B_{\eta}(m)(\gamma)$ for $\gamma=\xi$.

Conditions (b), (c), and (d) are immediate from the definition of $f_{\xi}$.

Condition (e) holds because $\left\langle\left\langle I_{\eta}, f_{\eta}, B_{\eta}\right\rangle: \eta<\xi\right\rangle$ is good and $\xi$ is a limit ordinal.

To prove (f) and (g) first observe that, by Lemma 3.29, for every $i<\omega$ we have $K\left(\xi, m_{i}\right)=K\left(\varepsilon_{i}, m_{i}\right) \cup\left\{\varepsilon_{i}\right\}$. This implies that $l_{\delta_{j}}\left(\varepsilon_{i}, m_{i}\right) \leq l_{\delta_{j}}\left(\xi, m_{i}\right)$ and $k_{\delta_{j}}\left(\xi, m_{i}\right)=k_{\delta_{j}}\left(\varepsilon_{i}, m_{i}\right)$ for every $j \leq i$, because $\varepsilon_{i}$ is a succesor ordinal. In particular, for every $j \leq i$ we have

$$
l_{\delta_{j}}\left(\xi, m_{i}\right)-k_{\delta_{j}}\left(\xi, m_{i}\right) \geq l_{\delta_{j}}\left(\varepsilon_{i}, m_{i}\right)-k_{\delta_{j}}\left(\varepsilon_{i}, m_{i}\right) .
$$


To see (f) fix an $m=m_{i} \in I_{\xi}$. We need to show that $l_{\delta^{*}}(\xi, m)>k_{\delta^{*}}(\xi, m)$. First assume that $\xi=\min \left(S_{\delta^{*}}\right)$. Then, $K(\xi, m) \subseteq \xi$ is disjoint with $S_{\delta^{*}}$, so $k_{\delta^{*}}(\xi, m)=\left|K(\xi, m) \cap S_{\delta^{*}}\right|=0$. On the other hand, condition (iii) implies that $\bigcup g_{\delta^{*}}[2] \subseteq K(\xi, m)$. So, $l_{\delta^{*}}(\xi, m) \geq 1>0=k_{\delta^{*}}(\xi, m)$. Next, consider the case when $\xi>\min \left(S_{\delta^{*}}\right)$. Then, $\delta^{*}=\delta_{i}$ for some $i<\omega$. Therefore, (ii) and (3.6) imply that

$$
l_{\delta^{*}}\left(\xi, m_{i}\right)-k_{\delta^{*}}\left(\xi, m_{i}\right) \geq l_{\delta_{i}}\left(\varepsilon_{i}, m_{i}\right)-k_{\delta_{i}}\left(\varepsilon_{i}, m_{i}\right)>i \geq 0 .
$$

Thus (f) holds.

To see (g) fix a $\delta<\omega_{1}$ such that $\bigcup \operatorname{rang}\left(g_{\delta}\right) \subseteq \xi$. We need to show that $\lim _{i \rightarrow \infty}\left(l_{\delta}\left(\xi, m_{i}\right)-k_{\delta}\left(\xi, m_{i}\right)\right)=\infty$. First assume that $\xi>\min \left(S_{\delta}\right)$. Then $\delta=\delta_{j}$ for some $j<\omega$. So, by (ii) and (3.6), we have that for all $i \geq j$

$$
l_{\delta}\left(\xi, m_{i}\right)-k_{\delta}\left(\xi, m_{i}\right)=l_{\delta_{j}}\left(\xi, m_{i}\right)-k_{\delta_{j}}\left(\xi, m_{i}\right) \geq l_{\delta_{j}}\left(\varepsilon_{i}, m_{i}\right)-k_{\delta_{j}}\left(\varepsilon_{i}, m_{i}\right) \geq i .
$$

This ensures that $(\mathrm{g})$ holds. Finally, assume that $\xi \leq \min \left(S_{\delta}\right)$. Then, for every $m<\omega$, we have $K(\xi, m) \cap S_{\delta}=\emptyset$ and so, $k_{\delta}(\xi, m)=0$. Thus, in this case it is enough to show that $\lim _{i \rightarrow \infty} l_{\delta}\left(\xi, m_{i}\right)=\infty$. But for every $l<\omega$ we have $\bigcup g_{\delta}[l+1] \subseteq \xi=\left\{\xi_{j}: j<\omega\right\}$. Thus, there exists an $i_{0}<\omega$ such that $\bigcup g_{\delta}[l+1] \subseteq \xi=\left\{\xi_{j}: j \leq i_{0}\right\}$. Since, by (iii), for every $i \geq i_{0}$ we have $\left\{\xi_{j}: j \leq i\right\} \subseteq K\left(\xi, m_{i}\right)$ we conclude that $\bigcup g_{\delta}[l+1] \subseteq K\left(\xi, m_{i}\right)$ for every $i \geq i_{0}$. Thus, $l_{\delta}\left(\xi, m_{i}\right) \geq l$ for every $i \geq i_{0}$ and $\operatorname{so} \lim _{i \rightarrow \infty} l_{\delta}\left(\xi, m_{i}\right)=\infty$.

Lemma 3.31 Let $\left\langle\left\langle I_{\eta}, f_{\eta}, B_{\eta}\right\rangle \in \mathcal{T}: \eta \leq \xi\right\rangle$ be a good sequence, $I \in\left[I_{\xi}\right]^{\omega}$, and let $\left\langle D_{m} \in \mathcal{P}\left(B_{\xi}(m)(\xi+1)\right) \cap \mathcal{D}: m \in I\right\rangle$ be arbitrary. Then, the sequence $\left\langle\left\langle I_{\eta}, f_{\eta}, B_{\eta}\right\rangle \in \mathcal{T}: \eta \leq \xi+1\right\rangle$ is good, where $\left\langle I_{\xi+1}, f_{\xi+1}, B_{\xi+1}\right\rangle \in \mathcal{T}$ is defined as

(i) $I_{\xi+1}=I$,

(ii) $f_{\xi+1}(m)= \begin{cases}D_{m} & \text { if } m \in I_{\xi+1} \\ B_{0}(m)(\xi+1) & \text { otherwise, }\end{cases}$

(iii) $B_{\xi+1}(m) \in\left(\mathcal{P}\left(f_{\xi+1}(m)\right) \cap \mathcal{D}\right)^{\omega_{1}}$ is any almost disjoint sequence for every $m<\omega$.

Proof. To show that (a) holds it is enough to check it only for the pair $\langle\eta, \xi+1\rangle$ in place of $\langle\zeta, \eta\rangle$. So, choose an $\eta<\xi+1$ and $m<\omega$. We need to show that either $f_{\xi+1}(m) \cap f_{\eta}(m)$ is finite, or there exists a $\gamma \leq \xi+1$ such that $f_{\xi+1}(m) \subseteq B_{\eta}(m)(\gamma)$. We consider several cases. 
$m \notin I_{\xi+1}$ : We will consider two subcases.

$\eta=0$ : Then $f_{\xi+1}(m)=B_{0}(m)(\xi+1)=B_{\eta}(m)(\gamma)$ for $\gamma=\xi+1$.

$\eta>0$ : Apply Remark 3.27 to find a $\gamma \leq \eta$ such that $f_{\eta}(m) \subseteq^{*} B_{0}(m)(\gamma)$. Since $f_{\xi+1}(m)=B_{0}(m)(\xi+1)$, we have that $\left|f_{\xi+1}(m) \cap f_{\eta}(m)\right|<\omega$.

$m \in I_{\xi+1}:$ We compare $\eta$ with $\xi$.

$\eta<\xi$ : By (a) either $\left|f_{\xi}(m) \cap f_{\eta}(m)\right|<\omega$ or there exists a $\gamma \leq \xi$ such that $f_{\xi}(m) \subseteq^{*} B_{\eta}(m)(\gamma)$. Since $f_{\xi+1}(m) \subseteq B_{\xi}(m)(\xi+1) \subseteq f_{\xi}(m)$ we have that $\left|f_{\xi+1}(m) \cap f_{\eta}(m)\right|<\omega$ or $f_{\xi+1}(m) \subseteq B_{\eta}(m)(\gamma)$.

$\eta=\xi$ : Clearly $f_{\xi+1}(m)=D_{m} \subseteq B_{\xi}(m)(\xi+1)=B_{\eta}(m)(\gamma)$ for $\gamma=\xi+1$.

This proves that (a) holds.

Conditions (b), (d), and (e) are obvious by the definition of $f_{\xi+1}$. Conditions (c) and (f) hold, since there are no new limit ordinals $\eta<\xi+1$.

To see that $(g)$ holds take a $\delta<\omega_{1}$ such that $\bigcup \operatorname{rang}\left(g_{\delta}\right) \subseteq \xi+1$. Then also $\bigcup \operatorname{rang}\left(g_{\delta}\right) \subseteq \xi$ since, by Fact 3.23, $\bigcup \operatorname{rang}\left(g_{\delta}\right)=\min \left(S_{\delta}\right)$ is a limit ordinal. Thus, $\lim _{\substack{m \in I_{\xi} \\ m \rightarrow \infty}}\left(l_{\delta}(\xi, m)-k_{\delta}(\xi, m)\right)=\infty$. Also, by Lemma 3.29 and the definition of $f_{\xi+1}^{m}(m)$ we have $K(\xi+1, m)=K(\xi, m) \cup\{\xi\}$ for every $m \in I_{\xi+1}$. This implies that $k_{\delta}(\xi+1, m) \leq k_{\delta}(\xi, m)+1$ and $l_{\delta}(\xi, m) \leq l_{\delta}(\xi+1, m)$ for every $m \in I_{\xi+1}$. So, $l_{\delta}(\xi+1, m)-k_{\delta}(\xi+1, m) \geq l_{\delta}(\xi, m)-k_{\delta}(\xi, m)-1$. Since $I_{\xi+1} \subseteq I_{\xi}$ is infinite, we have

$$
\lim _{\substack{m \in I_{\xi+1} \\ m \rightarrow \infty}}\left(l_{\delta}(\xi+1, m)-k_{\delta}(\xi+1, m)\right) \geq \lim _{\substack{m \in I_{\xi} \\ m \rightarrow \infty}}\left(l_{\delta}(\xi, m)-k_{\delta}(x i, m)-1\right)=\infty .
$$

So, $(h)$ holds.

Corollary 3.32 Let $\left\langle\left\langle I_{\eta}, f_{\eta}, B_{\eta}\right\rangle \in \mathcal{T}: \eta \leq \xi\right\rangle$ be good. If $P$ is a prism in $2^{\omega \times X}$, then there exists an $\left\langle I_{\xi+1}, f_{\xi+1}, B_{\xi+1}\right\rangle \in \mathcal{T}$, a suprism $Q$ of $P$, and an $i<2$ such that

(i) $\left\langle\left\langle I_{\eta}, f_{\eta}, B_{\eta}\right\rangle: \eta \leq \xi+1\right\rangle$ is good and

(ii) $g \uparrow U_{\xi+1}$ is constant equal to $i$ for every $g \in Q$. 
Proof. Apply Lemma 3.14 to the prism $P$, the set $I_{\xi}$, and the family $\left\{B_{\xi}(m)(\xi+1): m \in I_{\xi}\right\}$ to find a subprism $Q$ of $P$, a set $I_{\xi+1} \in\left[I_{\xi}\right]^{\omega}$, a sequence $\left\langle B_{m} \in \mathcal{P}(B(m)(\xi+1)) \cap \mathcal{J}^{+}: m \in I_{\xi+1}\right\rangle$, and an $i<2$ such that $g \uparrow B$ is constant equal to $i$, where $B=\bigcup\left\{\{m\} \times B_{m}: m \in I_{\xi+1}\right\}$. For every $m \in I_{\xi+1}$ choose $D_{m} \in \mathcal{P}\left(B_{m}\right) \cap \mathcal{D}$. Then, if we define $f_{\xi+1}$ and $B_{\xi+1}$ as in Lemma 3.31, $\left\langle\left\langle I_{\eta}, f_{\eta}, B_{\eta}\right\rangle: \eta \leq \xi+1\right\rangle$ is good and $g\left\lceil U_{\xi+1}\right.$ is constant equal to $i$.

Corollary 3.33 Let $X$ be a countably infinite set, $\mathcal{J} \subseteq \mathcal{P}(X)$ a $Q$-like ideal on $X,\left\langle\left\langle I_{\eta}, f_{\eta}, B_{\eta}\right\rangle \in \mathcal{T}: \eta \leq \xi\right\rangle$ be good, and $P$ be a prism on $\mathcal{P}_{\omega \times X}$. Then, there exists a $\left\langle I_{\xi+1}, f_{\xi+1}, B_{\xi+1}\right\rangle \in \mathcal{T}$ and a subprism $Q$ of $P$ such that

(i) $\left\langle\left\langle I_{\eta}, f_{\eta}, B_{\eta}\right\rangle: \eta \leq \xi+1\right\rangle$ is good and

(ii) $\left|z(k) \cap U_{\xi+1}\right| \leq 1$ for every $z \in Q$ and $k<\omega$.

Proof. Let $A=\bigcup\left\{\{m\} \times B_{\xi}(m)(\xi+1): m \in I_{\xi}\right\}$. Then $A \in \mathcal{K}^{+}$and, by Lemma $3.13, \mathcal{K}$ is $Q$-like. So, by Lemma 3.11, there is a subprism $Q$ of $P$ and a $B \in \mathcal{P}(A) \cap \mathcal{K}^{+}$such that $|z(k) \cap B| \leq 1$ for every $z \in Q$ and $k<\omega$. Let $I_{\xi+1}=\operatorname{supp}(B) \subseteq I_{\xi}$ and for every $m \in I_{\xi+1}$ choose $D_{m} \in \mathcal{P}\left((B)_{m}\right) \cap \mathcal{D}$. Then, if we define $f_{\xi+1}$ and $B_{\xi+1}$ as in Lemma 3.31, $\left\langle\left\langle I_{\eta}, f_{\eta}, B_{\eta}\right\rangle: \eta \leq \xi+1\right\rangle$ is good and $\left|z(k) \cap U_{\xi+1}\right| \leq 1$ for every $z \in Q$ and $k<\omega$.

Theorem 3.34 Let $X$ be a countably infinite set, $\mathcal{J} \subseteq \mathcal{P}(X)$ be a rich ideal, let $\mathcal{D} \subseteq \mathcal{J}^{+}$be a dense family, and put $\mathcal{K}=[\omega]^{<\omega} \otimes \mathcal{J}$. Then, $\mathrm{CPA}_{\text {prism }}^{\text {game }}$ implies that there exists an $\omega_{1}$-generated $\omega_{1}-O K$ point extending $\mathcal{F}_{\mathcal{K}}$ with a basis $\left\{U_{\xi}: \xi<\omega_{1}\right\} \subseteq \mathcal{D}^{*}$ which is not a $P$-point.

Proof. To define a triple $\left\langle I_{0}, f_{0}, B_{0}\right\rangle$ put $I_{0}=\omega$, for every $m<\omega$ define $f_{0}(m)=X$, and let $B_{0}(m)=\left\langle B_{0}(m)(\gamma): \gamma<\omega_{1}\right\rangle$ be an arbitrary $\omega_{1}{ }^{-}$ sequence of almost disjoint sets in $\mathcal{P}\left(f_{0}(m)\right) \cap \mathcal{D}$.

For a good sequence $\left\langle\left\langle I_{\eta}, f_{\eta}, B_{\eta}\right\rangle: \eta \leq \xi\right\rangle$ and a prism $P$ in $2^{\omega \times X}$ let us define a subprism $Q\left(\left\langle\left\langle I_{\eta}, f_{\eta}, B_{\eta}\right\rangle: \eta \leq \xi\right\rangle, P\right)=Q$ of $P$ and the triple $T\left(\left\langle\left\langle I_{\eta}, f_{\eta}, B_{\eta}\right\rangle: \eta \leq \xi\right\rangle, P\right)=\left\langle I_{\xi+1}, f_{\xi+1}, B_{\xi+1}\right\rangle \in \mathcal{T}$ as in Corollary 3.32. We define a strategy $S$ for Player II in the game $\operatorname{GAME}_{\text {prism }}\left(2^{\omega \times X}\right)$ as:

$$
S\left(\left\langle\left\langle P_{\eta}, Q_{\eta}\right\rangle: \eta<\xi\right\rangle, P_{\xi}\right)=Q\left(\left\langle\left\langle I_{\eta}, f_{\eta}, B_{\eta}\right\rangle: \eta \leq \xi\right\rangle, P_{\xi}\right),
$$

where $\left\langle\left\langle I_{\eta}, f_{\eta}, B_{\eta}\right\rangle: \eta \leq \xi\right\rangle$ is a good sequence defined by induction on $\eta \leq \xi$ as follows. Assume that $\left\langle\left\langle I_{\zeta}, f_{\zeta}, B_{\zeta}\right\rangle: \zeta<\eta\right\rangle$ is already defined. 
If $\eta=0$, then $\left\langle I_{\eta}, f_{\eta}, B_{\eta}\right\rangle=\left\langle I_{0}, f_{0}, B_{0}\right\rangle$ is defined as above.

If $\eta=\zeta+1$, then we put $\left\langle I_{\eta}, f_{\eta}, B_{\eta}\right\rangle=T\left(\left\langle\left\langle I_{\delta}, f_{\delta}, B_{\delta}\right\rangle: \delta \leq \zeta\right\rangle, P_{\zeta}\right)$.

If $\eta \in \Gamma$, then $\left\langle I_{\eta}, f_{\eta}, B_{\eta}\right\rangle$ is found using Lemma 3.30.

Notice that the sequence $\left\langle\left\langle I_{\zeta}, f_{\zeta}, B_{\zeta}\right\rangle: \zeta<\eta\right\rangle$ is good by the inductive hypothesis and Remark 3.25.

By $\mathrm{CPA}_{\text {prism }}^{\text {game }}$ strategy $S$ is not a winning strategy for Player II. So, there exists a game $\left\langle\left\langle P_{\xi}, Q_{\xi}\right\rangle: \xi<\omega_{1}\right\rangle$ played according to $S$ for which Player II loses, this is, $2^{\omega \times X}=\bigcup_{\xi<\omega_{1}} Q_{\xi}$. If $\left\langle\left\langle I_{\xi}, f_{\xi}, B_{\xi}\right\rangle \in \mathcal{T}: \xi<\omega_{1}\right\rangle$ is the sequence created when Player II uses strategy $S$, then this sequence is good by construction. Application of Lemma 3.28 to this sequence finishes the proof.

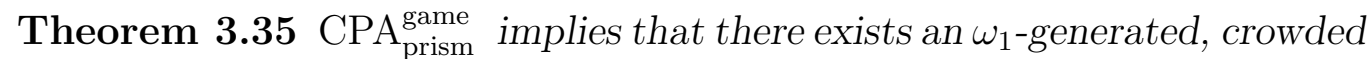
$\omega_{1}$-OK point on $\mathbb{Q}$ which is neither a $P$-point nor a $Q$-point.

Proof. The idea is to apply Theorem 3.34 to an apropriate ideal to get a crowded ultrafilter which is not a $Q$-point. Consider $X=\mathbb{Q} \times \omega$ with a natural product topology. Then, $X$ is homeomorphic to $\mathbb{Q}$. For every $m<\omega$ put $P_{m}=\left\{n<\omega: 2^{m}-1 \leq n<2^{m+1}-1\right\}$. Then $\left\{P_{m}: m<\omega\right\}$ is a partition of $\omega$ and $\left|P_{m}\right|=2^{m}$. For $A \subseteq \mathbb{Q} \times \omega$ put

$$
N_{A}(m)=\max \left\{k<\omega: \exists U \in \mathcal{I}_{S}^{+} \exists P \in\left[P_{m}\right]^{k} \quad U \times P \subseteq A\right\}
$$

and define $\mathcal{J} \subseteq \mathcal{P}(\mathbb{Q} \times \omega)$ as

$$
\mathcal{J}=\left\{A \subseteq \mathbb{Q} \times \omega: \varlimsup_{m \rightarrow \infty} N_{A}(m)<\infty\right\} .
$$

To see that $\mathcal{J}$ is closed under finite unions notice first that

$$
N_{A \cup B}(m) \leq N_{A}(m)+N_{B}(m) \text { for every } m<\omega \text { and } A, B \subseteq \mathbb{Q} \times \omega .
$$

Indeed, take a $P \subseteq P_{m}$ of cardinality $N_{A \cup B}(m)$ and $U \in \mathcal{I}_{S}^{+}$such that $U \times P \subseteq A \cup B$. Let $h: U \times P \rightarrow 2$ be a characteristic function of $A \cap(U \times P)$ and let $\varphi: U \rightarrow 2^{P}$ be defined by $\varphi(u)(p)=h(u, p)$. Since $2^{P}$ is finite, there exists a $g \in 2^{P}$ such that $V=\varphi^{-1}(g)$ belongs to $\mathcal{I}_{S}^{+}$. Let $P_{A}=g^{-1}(1)$ and $P_{B}=g^{-1}(0)$. Then $V \times P_{A} \subseteq A$ and $V \times P_{B} \subseteq B$. Therefore, $N_{A}(m) \geq\left|P_{A}\right|$ and $N_{B}(m) \geq\left|P_{B}\right|$. So, $N_{A \cup B}(m)=|P|=\left|P_{A}\right|+\left|P_{B}\right| \leq N_{A}(m)+N_{B}(m)$.

The above proved inequality easily implies that

$$
\varlimsup_{m \rightarrow \infty} N_{A \cup B}(m) \leq \varlimsup_{m \rightarrow \infty} N_{A}(m)+\varlimsup_{m \rightarrow \infty} N_{B}(m)
$$


for every and $A, B \subseteq \mathbb{Q} \times \omega$. Thus, $\mathcal{J}$ is closed under finite unions. Since it clearly is closed also under subsets, we can conclude that $\mathcal{J}$ is an ideal on $\mathbb{Q} \times \omega$ containing all the singletons. We will prove that

the ideal $\mathcal{J}$ is rich.

First notice how (3.7) implies the theorem. Since Perf $(\mathbb{Q})$ is dense in $\mathcal{I}_{S}^{+}$, it is easy to see that $\mathcal{D}=\operatorname{Perf}(\mathbb{Q} \times \omega)$ is dense in $\mathcal{J}^{+}$. Let $\mathcal{U}$ be an ultrafilter on $\omega \times X$ from Theorem 3.34 applied to $\mathcal{J}$ and $\mathcal{D}$. Since $X=\mathbb{Q} \times \omega$ is homeomorphic to $\mathbb{Q}$, so is $\omega \times X$ and $\mathcal{D}^{*}$ contains only its perfect subsets. Therefore, $\mathcal{U}$ can be considered as crowded. Moreover, a partition $\mathcal{P}=\left\{\{n\} \times\left(\{q\} \times P_{m}\right): q \in \mathbb{Q} \& n, m<\omega\right\}$ of $\omega \times X$ into finite sets does not admit partial selector in $\mathcal{U}$, since each such partial selector belongs to $\mathcal{K}=[\omega]^{<\omega} \times \mathcal{J}$. Thus, $\mathcal{U}$ is not a $Q$-point.

To prove property (3.7) fix an $A \in \mathcal{J}^{+}$. Then there exist $\left\langle m_{k} \in \omega: k<\omega\right\rangle$, $\left\{U_{k} \in \mathcal{I}_{S}^{+}: k<\omega\right\}$, and $\left\langle Q_{k} \subseteq P_{m_{k}}: k<\omega\right\rangle$ such that $U_{k} \times Q_{k} \subseteq A$ and $\left|Q_{k}\right|>k \cdot 2^{2^{k}}$ for every $k<\omega$.

First we prove condition (\#) from Definition 3.8. Since, by Lemma 3.12, the ideal $\mathcal{I}_{S}$ on $\mathbb{Q}$ is rich, for every $k<\omega$ there exists an almost disjoint family $\left\{U_{f}^{k}: f \in 2^{\omega}\right\} \subseteq \mathcal{P}\left(U_{k}\right) \cap \mathcal{I}_{S}^{+}$. Also, for every $k<\omega$ there exists a pairwise disjoint family $\left\{A_{s}: s \in 2^{k}\right\} \subseteq\left[Q_{k}\right]^{k}$. For $f \in 2^{\omega}$ define $A_{f}=$ $\bigcup\left\{U_{f}^{k} \times A_{f \mid k}: k<\omega\right\}$. Then, $\left\{A_{f}: f \in 2^{\omega}\right\} \subseteq \mathcal{P}(A) \cap \mathcal{J}^{+}$is almost disjoint, proving (\#).

To prove that $\mathcal{J}$ is prism-friendly let $P$ be a prism in $2^{X}$. If $P$ is singleton then condition $(\bullet)$ is clearly satisfied. So, assume that $P \in \operatorname{Perf}\left(2^{X}\right)$ and let $f$ be a witness function for it. By Remark 1.1 we can assume that $f$ is defined on $\mathfrak{C}^{\alpha}$ for some $0<\alpha<\omega_{1}$. Our first goal is to find a subprism $Q^{\prime}$ of $P$ and two sequences $\left\{V_{k} \subseteq U_{k}: k<\omega\right\} \subseteq \mathcal{I}_{S}^{+}$and $\left\{A_{k} \in\left[P_{m_{k}}\right]^{k}: k<\omega\right\}$ such that

$$
g \uparrow V_{k} \times A_{k} \text { is constant for every } g \in Q^{\prime} \text {. }
$$

For every $k<\omega$ define $\mathcal{D}_{k}$ as the set of all disjoint collections $\mathcal{E} \in\left[\mathbb{P}_{\alpha}\right]^{<\omega}$ such that there exists a $V_{\langle\mathcal{E}, k\rangle} \in \mathcal{P}\left(U_{k}\right) \cap \mathcal{I}_{S}^{+}$such that for every $q \in Q_{k}, E \in \mathcal{E}$, and $h, h^{\prime} \in E$, each $f(h)$ is constant on $V_{\langle\mathcal{E}, k\rangle} \times\{q\}$ and

$$
f(h)\left\lceil V_{\langle\mathcal{E}, k\rangle} \times\{q\}=f\left(h^{\prime}\right) \uparrow V_{\langle\mathcal{E}, k\rangle} \times\{q\} .\right.
$$

It is immediate that $\mathcal{D}_{k}$ is closed under refinaments. To prove that $\mathcal{D}_{k}$ satisfies the condition ( $\dagger$ ) from Proposition 1.3 let $\mathcal{E} \in \mathcal{D}_{k}$ and $E \in \mathbb{P}_{\alpha}$ be such 
that $E \cap \bigcup \mathcal{E}=\emptyset$. Let $\left\{q_{i}: i \leq r\right\}$ be an enumeration of $Q_{k}$. Using Proposition 3.8, construct inductively decreasing sequences $\left\langle E_{i} \in \mathbb{P}_{\alpha} \cap \mathcal{P}(E): i \leq r\right\rangle$, $\left\langle V_{i} \in \mathcal{P}\left(V_{\langle\mathcal{E}, k\rangle}\right) \cap \mathcal{I}_{S}^{+}: i \leq r\right\rangle$, and a sequence $\left\langle j_{i}<2: i \leq r\right\rangle$ such that for every $i \leq r$

$$
f(h) \uparrow V_{i} \times\left\{q_{i}\right\} \text { is constant equal to } j_{i} \text { for every } h \in E_{i} .
$$

Therefore, if we put $E^{\prime}=E_{r}$ and $V_{\left\langle\mathcal{E} \cup\left\{E^{\prime}\right\}, k\right\rangle}=V_{r}$, then $\mathcal{E} \cup\left\{E^{\prime}\right\} \in \mathcal{D}_{k}$ and condition $(\dagger)$ is satisfied. Thus, by Proposition 1.3, for every $k<\omega$ there exists a family $\mathcal{E}_{k}=\left\{E_{i}: i<2^{k}\right\} \in \mathcal{D}_{k}$ of pairwise disjoint sets with $E^{0}=\bigcap_{k<\omega} \cup \mathcal{E}_{k} \in \mathbb{P}_{\alpha}$. We will prove that $Q^{\prime}=f\left[E^{0}\right]$ satisfies (3.8) with $V_{k}=V_{\left\langle\mathcal{E}_{k}, k\right\rangle}$ and some sequence $\left\langle A_{k} \in\left[Q_{k}\right]^{k}: k<\omega\right\rangle$.

To see this fix $k<\omega$ and $v_{0} \in V_{k}=V_{\left\langle\mathcal{E}_{k}, k\right\rangle}$, and for each $i<2^{k}$ pick an $h_{i} \in E_{i} \in \mathcal{E}_{k}$. Define $\varphi_{k}: Q_{k} \rightarrow 2^{2^{k}}$ by $\varphi_{k}(p)(i)=f\left(h_{i}\right)\left(v_{0}, p\right)$. Since $\left|Q_{k}\right|>k \cdot 2^{2^{k}}$, there exists an $s_{k} \in 2^{2^{k}}$ such that $\left|\varphi_{k}^{-1}\left\{s_{k}\right\}\right| \geq k$. Pick an $A_{k} \in\left[\varphi_{k}^{-1}\left\{s_{k}\right\}\right]^{k}$. To see that the pair $\left\langle V_{k}, A_{k}\right\rangle$ satisfies (3.8), pick a $g \in Q^{\prime}$. Then there exists an $i<2^{k}$ and an $h \in E_{i} \in \mathcal{E}_{k}$ such that $g=f(h)$. We will show that $g\left[V_{k} \times A_{k}\right]=\left\{s_{k}(i)\right\}$.

Let $\langle v, q\rangle \in V_{k} \times A_{k}$. Since, by (3.9), $f(h)$ is constant on $V_{k} \times\{q\}$, we have $f(h)(v, q)=f(h)\left(v_{0}, h\right)$. Also, (3.9) gives $f(h)\left(v_{0}, q\right)=f\left(h_{i}\right)\left(v_{0}, q\right)$. Hence, $g(v, q)=f\left(h_{i}\right)\left(v_{0}, q\right)=\varphi_{k}(q)(i)=s_{k}(i)$. So, $g\left\lceil V_{k} \times A_{k}\right.$ is constant equal to $s_{k}(i)$ and (3.8) holds.

To finish the proof for every $k<\omega$ pick $\left\langle v_{k}, a_{k}\right\rangle \in V_{k} \times A_{k}$ and put $S=\left\{\left\langle v_{k}, a_{k}\right\rangle: k<\omega\right\}$. Let $\mathcal{I}=[X]^{<\omega}$. Then $\mathcal{I}$ is weakly selective and $S \in \mathcal{I}^{+}$. If we identify $2^{X}$ with $\mathcal{P}(X)$, then $Q^{\prime}$ can be treated as a prism in $\mathcal{P}(X)$. Since $[X]^{\omega}$ is residual in $\mathcal{P}(X)$, by Proposition 1.2 we can assume that $Q^{\prime}$ is a prism in $[X]^{\omega}$. So, by Proposition 3.8, there exist a subprism $Q$ of $Q^{\prime}$, a set $S_{0} \in[S]^{\omega}$, and an $i<2$ such that $g\left[S_{0}\right]=\{i\}$ for every $g \in Q$. Put $B=\bigcup\left\{V_{k} \times A_{k}:\left\langle v_{k}, a_{k}\right\rangle \in S_{0}\right\}$. Then, $g \uparrow B$ is constant equal $i$ for every $g \in Q$. It is clear that $B \subseteq A$. Since $V_{k} \times A_{k} \subseteq B$ and $A_{k} \in\left[P_{m_{k}}\right]^{k}$ we have $N_{B}\left(m_{k}\right) \geq k$. This implies that $\overline{\lim }_{m \rightarrow \infty} N_{B}(m)=\infty$ and that $B \in \mathcal{J}^{+}$. So, $Q$ and $B$ satisfy $(\bullet)$.

Theorem 3.36 Let $X$ be a countably infinite set, $\mathcal{J} \subseteq \mathcal{P}(X)$ a rich and $Q$-like ideal on $X$, and let $\mathcal{D} \subseteq \mathcal{J}^{+}$be dense. Then, $\mathrm{CPA}_{\text {prism }}^{\text {game implies that }}$ there exists an $\omega_{1}$-generated, crowded $\omega_{1}-O K$ point on $\omega \times X$ which is also a $Q$-point but not a $P$-point. 
Proof. This proof combines the elements of the proofs of Theorems 3.21 and 3.34. Let $\mathcal{Y}=\mathcal{P}_{\omega \times X} \cup 2^{\omega \times X}$ be as in Theorem 3.21.

For a good sequence $\bar{G}=\left\langle\left\langle I_{\eta}, f_{\eta}, B_{\eta}\right\rangle: \eta \leq \xi\right\rangle$ and a prism $P$ in $\mathcal{Y}$ let us define a subprism $Q(\bar{G}, P)$ of $P$ and a triple $T(\bar{G}, P) \in \mathcal{T}$ as follows.

- If $U \cap 2^{\omega \times X} \neq \emptyset$, then we can choose a subprism $P_{0} \subseteq 2^{\omega \times X}$ of $P$. The choice of $P_{0}$ is obvious if $P$ is a singleton, and it follows from Proposition 1.2, otherwise. Then we apply Corollary 3.32 to $\bar{G}$ and $P_{0}$ to find appropriate subprism $Q(\bar{G}, P)$ of $P_{0}$ and $\left\langle I_{\xi+1}, f_{\xi+1}, B_{\xi+1}\right\rangle \in \mathcal{T}$. We put $T(\bar{G}, P)=\left\langle I_{\xi+1}, f_{\xi+1}, B_{\xi+1}\right\rangle$.

- If $U \cap 2^{\omega \times X}=\emptyset$, then $P$ is a prism in $\mathcal{P}_{\omega \times X}$.

Then, we can use Corollary 3.33 to find appropriate $\left\langle I_{\xi+1}, f_{\xi+1}, B_{\xi+1}\right\rangle \in$ $\mathcal{T}$ and a subprism $Q(\bar{G}, P)$ of $P_{0}$. We put $T(\bar{G}, P)=\left\langle I_{\xi+1}, f_{\xi+1}, B_{\xi+1}\right\rangle$.

We define a strategy $S$ for Player II in the game $\operatorname{GAME}_{\text {prism }}(\mathcal{Y})$ as:

$$
S\left(\left\langle\left\langle P_{\eta}, Q_{\eta}\right\rangle: \eta<\xi\right\rangle, P_{\xi}\right)=Q\left(\left\langle\left\langle I_{\eta}, f_{\eta}, B_{\eta}\right\rangle: \eta \leq \xi\right\rangle, P_{\xi}\right),
$$

where the sequence $\left\langle\left\langle I_{\eta}, f_{\eta}, B_{\eta}\right\rangle: \eta \leq \xi\right\rangle$ is defined as in Theorem 3.34.

By $\mathrm{CPA}_{\text {prism }}^{\text {game }}$ strategy $S$ is not a winning strategy for Player II. So, there exists a game $\left\langle\left\langle P_{\xi}, Q_{\xi}\right\rangle: \xi<\omega_{1}\right\rangle$ played according to $S$ for which Player II loses, this is, $\mathcal{Y}=\bigcup_{\xi<\omega_{1}} Q_{\xi}$. If $\left\langle\left\langle I_{\xi}, f_{\xi}, B_{\xi}\right\rangle \in \mathcal{T}: \xi<\omega_{1}\right\rangle$ is the sequence created when Player II uses strategy $S$, then, by Remark 3.25, this sequence is good.

If $g \in 2^{\omega \times X}$, then there exists a $\xi<\omega_{1}$ such that $g \in Q_{\xi}$. Therefore, $Q_{\xi} \subseteq 2^{\omega \times X}$ and $g \uparrow U_{\xi+1}$ is constant. Thus, by Lemma 3.28, the family $\left\{U_{\xi}: \xi<\omega_{1}\right\}$ forms a base for a non-principal ultrafilter $\mathcal{U}$ on $\omega \times X$ which is an $\omega_{1}$-OK point but not a $P$-point. Note that $\left\{U_{\xi}: \xi<\omega_{1}\right\} \subseteq \mathcal{D}^{*}$. To see that $\mathcal{U}$ is a $Q$-point, take a $z \in \mathcal{P}_{\omega \times X}$. Then, there exists a $\xi<\omega_{1}$ such that $z \in Q_{\xi}$. This means that $Q_{\xi} \subseteq \mathcal{P}_{\omega \times X}$ and that $\left|z(k) \cap U_{\xi+1}\right| \leq 1$ for every $k<\omega$. Hence, $\mathcal{U}$ is also a $Q$-point.

Corollary 3.37 $\mathrm{CPA}_{\text {prism }}^{\text {game }}$ implies that there is an $\omega_{1}$-generated, crowded $\omega_{1}$-OK point on $\omega \times X$ which is also a $Q$-point but not a $P$-point.

Proof. Apply Theorem 3.36 with $X=\mathbb{Q}, \mathcal{J}=\mathcal{I}_{S}$, and $\mathcal{D}=\operatorname{Perf}(\mathbb{Q})$. 


\subsection{Crowded $Q$-points with a large character}

In this section we prove under $\mathrm{CPA}_{\text {prism }}^{\text {game }}$ that there are $2^{\mathfrak{c}}$-many crowded $Q$ points on $\mathbb{Q}$ of character $\mathfrak{c}$. This result contrasts with the following theorem.

Proposition 3.38 (K. Ciesielski and J. Pawlikowski, [9, Corollary 2.7]) Axiom $\mathrm{CPA}_{\text {cube }}$ implies that every selective ultrafilter has character $\omega_{1}$.

Let $X$ be a countably infinite set, let $\left\langle\mathcal{U}_{n}: n<\omega\right\rangle$ be a sequence of ultrafilters on $X$, and let $\mathcal{V}$ be an ultrafilter on $\omega$. We define an ultrafilter $\sum_{\mathcal{V}} \mathcal{U}_{n}$ on $\omega \times X$ as follows

$$
U \in \sum_{\mathcal{V}} \mathcal{U}_{n} \Longleftrightarrow\left\{n<\omega:(U)_{n} \in \mathcal{U}_{n}\right\} \in \mathcal{V}
$$

Lemma 3.39 (Folklore) Let $\left\langle\mathcal{U}_{n}: n<\omega\right\rangle$ be a sequence of ultrafilters on $X$ and let $\mathcal{V}$ be an ultrafilter on $\omega$. If $\chi\left(\sum_{\mathcal{V}} \mathcal{U}_{n}\right)<\kappa$, then $\chi(\mathcal{V})<\kappa$.

Proof. Let $\chi\left(\sum_{\mathcal{V}} \mathcal{U}_{n}\right)=\lambda<\kappa$ and let $\left\{U_{\alpha}: \alpha<\lambda\right\}$ be a basis for $\sum_{\mathcal{V}} \mathcal{U}_{n}$. Then, $V_{\alpha}=\left\{n<\omega:\left(U_{\alpha}\right)_{n} \in \mathcal{U}_{n}\right\} \in \mathcal{V}$ for every $\alpha<\lambda$. Our goal is to prove that $\left\{V_{\alpha}: \alpha<\lambda\right\}$ is a basis for $\mathcal{V}$. If $V \in \mathcal{V}$ then $U_{V}=$ $\bigcup\{\{n\} \times X: n \in V\} \in \sum_{\mathcal{V}} \mathcal{U}_{n}$. Therefore, there exists an $\alpha<\lambda$ such that $U_{\alpha} \subseteq U_{V}$. This implies that if $n \in V_{\alpha}$, then $\{n\} \times\left(U_{\alpha}\right)_{n} \subseteq U_{\alpha} \subseteq U_{V}$. Hence, $n \in V$ and $V_{\alpha} \subseteq V$.

Corollary 3.40 If $\chi(\mathcal{V})=\mathfrak{c}$, then $\chi\left(\sum_{\mathcal{V}} \mathcal{U}_{n}\right)=\mathfrak{c}$.

Proposition 3.41 (B. Pospiśíl [40], see [24]) If $X$ is an infinite set, then there exist $2^{2^{|X|}}$-many non-principal ultrafilters of character $2^{|X|}$ on $X$.

Consider the space $[\mathbb{Q}]^{\omega}$ with the same topology as the classical Polish space $[\omega]^{\omega}$ upon natural identification. Observe that if $n<\omega$ is arbitrary, then $\{n\} \times[\mathbb{Q}]^{\omega}$ is homeomorphic to $[\mathbb{Q}]^{\omega}$. Put $\mathcal{X}_{n}=\{n\} \times[\mathbb{Q}]^{\omega}$ for every $n<\omega$ and let $\mathcal{Z}=\bigcup_{n<\omega} \mathcal{X}_{n} \cup \mathcal{P}_{\omega \times \mathbb{Q}}$ be the topological sum of these spaces. Then, $\mathcal{Z}$ is a Polish space and $A \subseteq \mathcal{Z}$ is open if and only if $A \cap \mathcal{X}_{n}$ is open in $\mathcal{X}_{n}$ and $A \cap \mathcal{P}_{\omega \times \mathbb{Q}}$ is open in $\mathcal{P}_{\omega \times \mathbb{Q}}$ (see [26]). Also, given any prism $P \in \operatorname{Perf}(\mathcal{Z})$ either there exists an $n<\omega$ such that $P \cap \mathcal{X}_{n}$ is non-meager in $\mathcal{X}_{n}$ or $P \cap \mathcal{P}_{\omega \times \mathbb{Q}}$ is non-meager in $\mathcal{P}_{\omega \times \mathbb{Q}}$. Hence, by Proposition 1.2 , if $P \in \operatorname{Perf}(\mathcal{Z})$ is a prism we can always assume that either there exists an 
$n<\omega$ such that $P \in \operatorname{Perf}\left(\mathcal{X}_{n}\right)$ or that $P \in \operatorname{Perf}\left(\mathcal{P}_{\omega \times \mathbb{Q}}\right)$ and that $P$ is a prism in $\mathcal{X}_{n}$ or $\mathcal{P}_{\omega \times \mathbb{Q}}$, respectively.

Let $p \in \mathbb{R} \backslash \mathbb{Q}$ be an irrational number and for a family $\mathcal{D} \subseteq \mathcal{P}(\mathbb{Q})$ let $F_{p}(\mathcal{D})=F(\mathcal{D})$ denote the filter generated by the family $\mathcal{D} \cup\left\{I_{n} \cap \mathbb{Q}: n<\omega\right\}$, where $I_{n}=\left[p-2^{-n}, p+2^{-n}\right]$.

Proposition 3.42 (K. Ciesielski, J. Pawlikowski [9, Lemma 4.23]) Let $\mathcal{D} \subseteq \operatorname{Perf}(\mathbb{Q})$ be a countable family such that $F(\mathcal{D})$ is non-trivial. Then for every prism $P$ in $[\mathbb{Q}]^{\omega}$ there exist a subprism $Q$ of $P$ and a $Z \in \operatorname{Perf}(\mathbb{Q})$ such that $F(\mathcal{D} \cup\{Z\})$ is non-trivial and either

(i) $Z \cap x=\emptyset$ for every $x \in Q$, or else

(ii) $Z \subseteq x$ for every $x \in Q$.

Lemma 3.43 Let $\mathcal{D} \subseteq \operatorname{Perf}(\mathbb{Q})$ be a countable family such that $F(\mathcal{D})$ is crowded. Then, there are the sequences $\left\langle J_{k}: k<\omega\right\rangle$ of pairwise disjoint clopen intervals in $\mathbb{Q}$ and $\left\langle S_{k} \subseteq J_{k}: k<\omega\right\rangle$ of perfect subsets of $\mathbb{Q}$ such that if $S=\bigcup_{k<\omega} S_{k}$, then for every $D \in F(\mathcal{D})$ there exists an $n<\omega$ such that $S \cap I_{n} \in \mathcal{P}(D) \cap \operatorname{Perf}(\mathbb{Q})$. Moreover, if $W \subseteq S$ is dense in $S$ then there exists a $Z \in \mathcal{P}(W) \cap \operatorname{Perf}(\mathbb{Q})$ such that $F(\mathcal{D} \cup\{Z\})$ is crowded.

Proof. Observe that since $F(\mathcal{D})$ is crowded it is possible to find a sequence $\left\langle D_{n} \in \operatorname{Perf}(\mathbb{Q}): n<\omega\right\rangle$ coinitial in $F(\mathcal{D})$ such that $D_{n+1} \subseteq D_{n} \subseteq I_{n}$ for every $n<\omega$. Define two sequences $\left\langle n_{k}: k<\omega\right\rangle$ and $\left\langle S_{k} \in \operatorname{Perf}(\mathbb{Q}): k<\omega\right\rangle$ such that $S_{k} \subseteq D_{k} \cap I_{n_{k}} \cap J_{k}$, where $J_{k}$ is a clopen interval such that $p \notin \overline{J_{k}}$. If $n_{k}$ and $S_{k}$ are already defined pick $n_{k+1}>n_{k}$ with $J_{k} \cap I_{n_{k+1}}=\emptyset$. Since $D_{k+1} \cap I_{n_{k+1}} \in F(\mathcal{D})$ and $F(\mathcal{D})$ is crowded we can find a clopen interval $J_{k+1}$ such that $p \notin \overline{J_{k+1}}$ and $J_{k+1} \cap D_{k+1} \cap I_{n_{k+1}} \neq \emptyset$. Define $S_{k+1}=J_{k+1} \cap D_{k+1} \cap I_{n_{k+1}}$. Then, $S_{k+1} \in \operatorname{Perf}(\mathbb{Q})$ and $S_{k+1} \subseteq D_{k+1} \cap I_{n_{k+1}}$. Now, if $S=\bigcup_{k<\omega} S_{k}$, then $\bigcup_{i>k} S_{i} \in \operatorname{Perf}(\mathbb{Q})$ and $S \cap I_{n_{k}}=\bigcup_{i>k} S_{i} \cap I_{n_{k}} \subseteq$ $\bigcup_{i>k} S_{i} \subseteq D_{k}$. This proves the first part of the lemma.

To prove the second part suppose that $W$ is dense in $S$. Since $S_{k} \in$ $\operatorname{Perf}(\mathbb{Q})$ for every $n<\omega$ we conclude that $W \cap S_{k}$ is non-scattered and contains a subset $Z_{k}$ from $\operatorname{Perf}(\mathbb{Q})$ for every $k<\omega$. Hence, if we put $Z=$ $\bigcup_{k<\omega} Z_{k}$ then, $Z \in \operatorname{Perf}(\mathbb{Q})$, and $Z \cap I_{n_{k}} \subseteq D_{k}$ for every $k<\omega$. This also shows that $F(\mathcal{D} \cup\{Z\})$ is crowded because $\left\langle D_{k} \in \operatorname{Perf}(\mathbb{Q}): k<\omega\right\rangle$ is coinitial in $F(\mathcal{D})$ and $Z \cap I_{n_{k}} \in \operatorname{Perf}(\mathbb{Q})$ for every $k<\omega$. 
Lemma 3.44 Let $\left\langle\mathcal{D}_{n} \in[\operatorname{Perf}(\mathbb{Q})]^{\leq \omega}: n<\omega\right\rangle$ be such that $F\left(\mathcal{D}_{n}\right)$ is crowded for every $n<\omega$ and let $P \in \operatorname{Perf}\left(\mathcal{P}_{\omega \times \mathbb{Q}}\right)$ be a prism. Then, there exist a sequence of sets $\left\langle W_{n} \in \operatorname{Perf}(\mathbb{Q}): n<\omega\right\rangle$ and a subprism $Q$ of $P$ such that $F\left(\mathcal{D}_{n} \cup\left\{W_{n}\right\}\right)$ is crowded and $|W \cap z(k)| \leq 1$ for every $z \in Q$ and $k<\omega$, where $W=\bigcup\left\{\{n\} \times W_{n}: n<\omega\right\}$.

Proof. For each $n<\omega$, let $S_{n} \in \operatorname{Perf}(\mathbb{Q})$ be the set found in Lemma 3.43 associated to $F\left(\mathcal{D}_{n}\right)$. Let $\mathcal{B}$ a countable basis for the topology on $\mathbb{Q}$ consisting of clopen sets. Consider the family $\mathcal{B}_{n}^{*}=\left\{B \cap S_{n}: B \in \mathcal{B} \&\left|B \cap S_{n}\right|=\omega\right\}$ for every $n<\omega$. Hence, $\mathcal{B}_{n}^{*} \in\left[\mathcal{I}_{S}^{+}\right]^{\omega}$ for every $n<\omega$. We apply Lemma 3.11 to the ideal $\mathcal{I}_{S}(\omega \times \mathbb{Q})$, the family $\left\{\{n\} \times V: V \in \mathcal{B}_{n}^{*} \& n<\omega\right\}$, and the prism $P$ to obtain a family $\mathcal{W}=\left\{\{n\} \times W^{*}: W^{*} \in \mathcal{P}(V) \cap \mathcal{I}_{S}^{+}, V \in \mathcal{B}_{n}^{*} \& n<\omega\right\}$ and a subprism $Q$ of $P$ such that $|\bigcup \mathcal{W} \cap z(k)| \leq 1$ for every $k<\omega$ and $z \in Q$. If we put $W_{n}^{*}=\bigcup\left\{W^{*} \in \mathcal{W}: W^{*} \subseteq S_{n}\right\}$ then, $W_{n}^{*}$ is dense in $S_{n}$ for every $n<\omega$. By the second part of Lemma 3.43 it is possible to find a $W_{n} \in \operatorname{Perf}(\mathbb{Q}) \cap \mathcal{P}\left(W_{n}^{*}\right)$ such that $F\left(\mathcal{D}_{n} \cup\left\{W_{n}\right\}\right)$ is crowded. Therefore, $Q$ and $\left\langle W_{n} \in \operatorname{Perf}(\mathbb{Q}): n<\omega\right\rangle$ are as required.

Theorem 3.45 Axiom $\mathrm{CPA}_{\text {prism }}^{\text {game }}$ implies that there is a sequence $\left\langle\mathcal{U}_{n}: n<\omega\right\rangle$ of ultrafilters on $\mathbb{Q}$ such that $\sum_{\mathcal{V}} \mathcal{U}_{n}$ is a crowded $Q$-point on $\omega \times \mathbb{Q}$ for every non-principal ultrafilter $\mathcal{V}$ on $\omega$. Therefore, there are $2^{\mathfrak{c}}$ crowded $Q$-points of character $\mathfrak{c}$.

Proof. For a prism $P \in \operatorname{Perf}(\mathcal{Z})$ and a sequence $\overline{\mathcal{D}}=\left\langle\mathcal{D}_{n}: n<\omega\right\rangle$ of countable families for which the filters $F\left(\mathcal{D}_{n}\right)$ are crowded we define $Q(\overline{\mathcal{D}}, P)$ and $\left\langle W_{n}: n<\omega\right\rangle_{\langle\overline{\mathcal{D}}, P\rangle}$ as follows.

If $P \in \operatorname{Perf}\left(\left\{n_{0}\right\} \times[\mathbb{Q}]^{\omega}\right)$ for some $n_{0}<\omega$ let $Z=Z\left(\mathcal{D}_{n_{0}}, P\right)$ and $Q\left(\mathcal{D}_{n_{0}}, P\right)$ be as in Proposition 3.42. Define $\left\langle W_{n}: n\langle\omega\rangle_{\langle\overline{\mathcal{D}}, P\rangle}\right.$ by putting $W_{n_{0}}=Z=Z\left(\mathcal{D}_{n_{0}}, P\right)$ and $W_{n}=\mathbb{Q}$ provided $n \neq n_{0}$ and $Q(\overline{\mathcal{D}}, P)=$ $Q\left(\mathcal{D}_{n_{0}}, P\right)$.

If $P \in \operatorname{Perf}\left(\mathcal{P}_{\omega \times \mathbb{Q}}\right)$ let $\left\langle W_{n}: n<\omega\right\rangle_{\langle\overline{\mathcal{D}}, P\rangle}$ and $Q(\overline{\mathcal{D}}, P)$ be the sequence and the subprism of $P$ as in Lemma 3.44. We define a strategy $S$ for Player II in the game $\operatorname{GAME}_{\text {prism }}(\mathcal{Z})$ as:

$$
S\left(\left\langle\left\langle P_{\eta}, Q_{\eta}\right\rangle: \eta<\xi\right\rangle, P_{\xi}\right)=Q\left(\overline{\mathcal{D}}_{\xi}, P_{\xi}\right)
$$

The sequence $\overline{\mathcal{D}}_{\xi}$ is defined inductively as $\overline{\mathcal{D}}_{\xi}=\left\langle\mathcal{D}_{n}^{\xi}: n<\omega\right\rangle$, where $\mathcal{D}_{n}^{\xi}=$ $\left\{\{n\} \times W_{n}^{\eta}: \eta<\xi\right\}$ and $\left\langle W_{n}^{\eta}: n<\omega\right\rangle=\left\langle W_{n}: n<\omega\right\rangle_{\left\langle\overline{\mathcal{D}}_{\eta}, P_{\eta}\right\rangle}$. 
By $\mathrm{CPA}_{\text {prism }}^{\text {game }}$ strategy $S$ is not a winning strategy for Player II. So, there exists a game $\left\langle\left\langle P_{\xi}, Q_{\xi}\right\rangle: \xi<\omega_{1}\right\rangle$ played according to $S$ for which Player II loses, this is, $\mathcal{Z}=\bigcup_{\xi<\omega_{1}} Q_{\xi}$ for every $\eta<\xi$.

For every $n<\omega$ define $\mathcal{U}_{n}=\bigcup_{\xi<\omega_{1}} F\left(\mathcal{D}_{n}^{\xi}\right)$. Notice that $\mathcal{U}_{n}$ is a crowded filter because it is the union of a chain of crowded filters. In particular, $\mathcal{U}_{n}$ is non-principal. To see that $\mathcal{U}_{n}$ is an ultrafilter pick any $A \in \mathcal{P}(\{n\} \times \mathbb{Q})$. Then either $A$ or $(\{n\} \times \mathbb{Q}) \backslash \mathbb{Q}$ is infinite so there exists a $\xi<\omega_{1}$ such that either $A \in Q_{\xi}$ or $(\{n\} \times \mathbb{Q}) \backslash A \in Q_{\xi}$. Suppose that $A \in Q_{\xi}$. Then, either $A \subseteq W_{n}^{\xi}$ or $A \cap W_{n}^{\xi}=\emptyset$. Therefore, either $A \in \mathcal{U}_{n}$ or $(\{n\} \times \mathbb{Q}) \backslash A \in \mathcal{U}_{n}$. The other case is similar. Hence, each $\mathcal{U}_{n}$ is a crowded ultrafilter on $\{n\} \times \mathbb{Q}$. Now pick any ultrafilter $\mathcal{V}$ on $\omega$ such that $\chi(\mathcal{V})=\mathfrak{c}$. We need to prove that $\mathcal{W}=\sum_{\mathcal{V}} \mathcal{U}_{n}$ is a $Q$-point. If $z \in \mathcal{P}_{\omega \times \mathbb{Q}}$, then there exists a $\xi<\omega$ such that $z \in Q_{\xi}$. Therefore, $\left|W_{\xi} \cap z(k)\right| \leq 1$ for every $k<\omega$, where $W_{\xi}=\bigcup\left\{\{n\} \times W_{n}^{\xi}: n<\omega\right\}$. Since $\{n\} \times\left(W_{\xi}\right)_{n}=\{n\} \times W_{n}^{\xi} \in \mathcal{U}_{n}$ for every $n<\omega$ it follows that $W_{\xi} \in \mathcal{W}$. For the last part use Proposition 3.41. 


\section{Chapter 4}

\section{Separately nowhere constant functions}

\subsection{Introduction}

For a topological space $\mathcal{X}$ a function $f: \mathcal{X} \rightarrow \mathcal{Y}$ is nowhere constant if $f$ is not constant on any non-empty open subset of $\mathcal{X}$. For a subset $G$ of a product space $\mathcal{X}=\prod_{i \in I} \mathcal{X}_{i}$ we say that a function $f: G \rightarrow \mathcal{Y}$ is separately nowhere constant if for every $t \in G$ and $k \in I$ function $f$ restricted to the section $G_{k}^{t}=\{x \in G: x \uparrow I \backslash\{k\}=t \uparrow I \backslash\{k\}\}$ is nowhere constant. This notion is the most natural when $G=\mathcal{X}$. In this case it is related in a natural way to the notion of a separately continuous function $f: \mathcal{X} \rightarrow \mathcal{Y}$, that is, such that $f$ restricted to every section $\mathcal{X}_{k}^{t}$ is continuous.

Note that every separately nowhere constant function is nowhere constant. However, the converse implication is false, as shown by the polynomial functions from $\mathbb{R}^{2}$ into $\mathbb{R}$ defined by $w_{0}(x, y)=x y$ and $w_{1}(x, y)=x$. This implications pattern stays in contrast with the implications for separate continuity: continuity implies separate continuity, but the converse implication is false.

We will consider the notion of being separately nowhere constant only for the product of perfect Polish spaces which we define here as a complete separable metric spaces without isolated points. (No function is nowhere constant if it is defined on a space containing isolated points.) Our main theorem on separately nowhere constant functions is the following result, where a subset $P$ of $\prod_{i \in I} \mathcal{X}_{i}$ is a perfect cube provided it is of the form 
$P=\prod_{i \in I} P_{i}$ with $P_{i} \in \operatorname{Perf}\left(\mathcal{X}_{i}\right)$ for all $i \in I$.

Theorem 4.1 Let $G$ be a dense $G_{\delta}$ subset of a product $\prod_{i \in I} \mathcal{X}_{i}$ of Polish spaces and let $f$ be a continuous function from $G$ into a Polish space $\mathcal{Y}$. If $f$ is separately nowhere constant, then there is a perfect cube $P$ in $\prod_{i \in I} \mathcal{X}_{i}$ such that $P \subseteq G$ and $f$ restricted to $P$ is one-to-one.

It is not difficult to see that the conclusion of the theorem remains true for the function $w_{0}(x, y)=x y$, despite the fact that $w_{0}$ is not separately nowhere constant. On the other hand, the theorem's conclusion is false for the nowhere constant function $w_{1}(x, y)=x$.

The theorem will be used to provide the examples distinguishing between the notions of $n$-cube densities.

Recall that if $0<n \leq \omega$, we say that a family $\mathcal{F} \subseteq \operatorname{Perf}(\mathcal{X})$ is $n$-cube dense provided that for every continuous injection $f: \mathfrak{C}^{n} \rightarrow \mathcal{X}$ there is a perfect cube $C \subseteq \mathfrak{C}^{n}$ such that $f[C] \in \mathcal{F}$ and that $\mathcal{F} \subseteq \operatorname{Perf}(\mathcal{X})$ is $\alpha$-prism dense provided that for every continuous injection $f: \mathfrak{C}^{\alpha} \rightarrow \mathcal{X}$ there is a $P \in \mathbb{P}_{\alpha}$ such that $f[P] \in \mathcal{F}$. If $\mathcal{F}$ is $\alpha$-prism dense for every $\alpha<\omega_{1}$ then we simply say that $\mathcal{F}$ is prism-dense.

To put these notions in a better perspective notice that 1-cube density is just the standard perfect set density, that is, $\mathcal{F} \subseteq \operatorname{Perf}(\mathcal{X})$ is 1 -cube dense provided every perfect subset of $X$ contains a set from $\mathcal{F}$.

To see that in general $n$-cube density is a stronger notion recall the following example, which is extracted from Miller's construction [39, thm. 5.10] of a Marczewski $s_{0}$-set of cardinality continuum.

Example $4.2[12,10]$ Let $\mathcal{X}=\mathfrak{C} \times \mathfrak{C}$ and let $\mathcal{E}$ be the family of all $P \in$ $\operatorname{Perf}(\mathcal{X})$ such that either all vertical sections of $P$ are countable, or all horizontal sections of $P$ are countable. Then $\mathcal{E}$ is dense in $\operatorname{Perf}(\mathcal{X})$ but it is not 2-cube dense.

The next two theorems discuss these notions of density. In particular, the first of them shows that essentially all these notions are different. The second theorem shows that any strengthening of the axiom $\mathrm{CPA}_{\text {prism }}$ obtained by replacing the prism density with a proper subclass of the densities we consider lead to the statement which is false in ZFC.

Theorem 4.3 For a Polish space $\mathcal{X}$, a family $\mathcal{F} \subseteq \operatorname{Perf}(\mathcal{X})$, and $1<\alpha<\omega_{1}$ consider the following sentences: 
$\mathrm{C}_{\alpha}$ family $\mathcal{F}$ is $\beta$-cube dense for every $0<\beta<\alpha$;

$\mathrm{P}_{\alpha}$ family $\mathcal{F}$ is $\beta$-prism dense for every $0<\beta<\alpha$.

Then, for $2<m<n<\omega$ and $\omega+1<\alpha<\gamma<\omega_{1}$, they are related by the following implications.

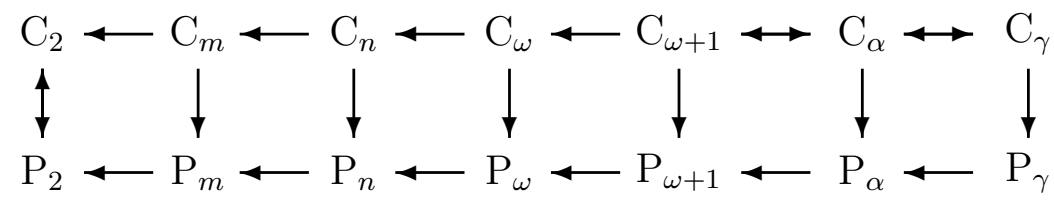

Moreover, none of these implications can be reversed.

For $\alpha<\omega_{1}$ we say that a family $\mathcal{F} \subseteq \operatorname{Perf}(\mathcal{X})$ is $\alpha$-prism* dense provided $\mathcal{F}$ is $\beta$-prism dense for every $0<\beta<\alpha$ and it is $n$-cube dense for every $0<n<\omega$.

Theorem 4.4 For every $\alpha<\omega_{1}$ and for every Polish space $\mathcal{X}$ there is an $\alpha$-prism* dense family $\mathcal{F} \subseteq \operatorname{Perf}(\mathcal{X})$ for which $|\mathcal{X} \backslash \bigcup \mathcal{F}|=\mathfrak{c}$.

These theorems will be proved in Section 4.3.

\subsection{Separately nowhere constant functions}

The space $\mathbb{R}^{\omega}$ is the product space of countably many copies of $\mathbb{R}$ with its usual topology. This is a Polish space and a vector space over $\mathbb{R}$ with the operations defined pointwise from the usual operations in $\mathbb{R}$. In this context we consider for every $k<\omega$ the canonical unit vectors $\vec{e}_{k} \in \mathbb{R}^{\omega}$ : $\vec{e}_{k}(k)=1$ and $\vec{e}_{k}(i)=0$ for all other $i<\omega$. If $S \subseteq \mathbb{R}^{\omega}$ and $\delta \in \mathbb{R}$ then $\delta \cdot \vec{e}_{k}+S=\left\{\delta \cdot \vec{e}_{k}+s: s \in S\right\}$. If $\varepsilon>0$ and $x \in \mathbb{R}^{\omega}$ then $B(x, \varepsilon)$ denotes the open ball with center $x$ and radius $\varepsilon$ and $\bar{B}(x, \varepsilon)$ is the corresponding closed ball. If $\mathcal{X}$ is a Polish space and $A \subseteq \mathcal{X}$, the closure of $A$ is denoted by $\bar{A}$. If $m<\omega$ then we identify $\mathbb{R}^{\omega}$ with $\mathbb{R} \times \mathbb{R}^{\omega \backslash\{m\}}$; if $y \in \mathbb{R}^{\omega \backslash\{m\}}$ and $G \subseteq \mathbb{R}^{\omega}$ then the section of $G$ along $y$ is the set $(G)^{y}=\{x \in \mathbb{R}:\langle x, y\rangle \in G\}$.

The following variant of Kuratowski-Ulam theorem will be useful in what follows. Although it looks like it should be well known, we could not locate it in the literature. Thus, we present it here with a proof. 
Lemma 4.5 Let $\mathcal{X}$ be a Polish space and consider $\mathcal{X}^{T}$ with the product topology, where $T \neq \emptyset$ is an arbitrary set. Fix at most countable family $\mathcal{K}$ of sets $K \subsetneq T$. Then for every comeager set $H \subseteq \mathcal{X}^{T}$ there exists a comeager set $G \subseteq H$ such that for every $x \in G$ and $K \in \mathcal{K}$ the set

$$
G_{x \uparrow K}=\left\{y \in \mathcal{X}^{T \backslash K}:(x \uparrow K) \cup y \in G\right\}
$$

is comeager in $\mathcal{X}^{T \backslash K}$.

Proof. Let $\left\{K_{i}: i<\omega\right\}$ be an enumeration of $\mathcal{K}$ with infinite repetitions. We construct, by induction on $i<\omega$, a decreasing sequence $\left\langle G_{i}: i<\omega\right\rangle$ of comeager subsets of $H$ such that for every $i<\omega$

(i) the set $\left(G_{i}\right)_{x \uparrow K_{i}}$ is comeager in $\mathcal{X}^{T \backslash K_{i}}$ for every $x \in G_{i}$.

Put $G_{-1}=H$ and assume that for some $i<\omega$ the comeager set $G_{i-1}$ is already constructed. To define $G_{i}$ identify $\mathcal{X}^{T}$ with $\mathcal{X}^{K_{i}} \times \mathcal{X}^{T \backslash K_{i}}$. Then, by Kuratowski-Ulam theorem, the set

$$
A=\left\{y \in \mathcal{X}^{K_{i}}:\left(G_{i-1}\right)_{y} \text { is comeager in } \mathcal{X}^{T \backslash K_{i}}\right\}
$$

is comeager in $\mathcal{X}^{K_{i}}$. Put $G_{i}=G_{i-1} \cap\left(A \times \mathcal{X}^{T \backslash K_{i}}\right)$.

Clearly $G_{i} \subseteq G_{i-1}$ is a comeager subset of $\mathcal{X}^{T}$. If $x \in G_{i}$ then $x \nmid K_{i} \in A$ so $\left(G_{i}\right)_{x \uparrow K_{i}}=\left(G_{i-1}\right)_{x \uparrow K_{i}}$ is comeager in $\mathcal{X}^{T \backslash K_{i}}$. So, (i) holds. This completes the definition of the sequence $\left\langle G_{i}: i<\omega\right\rangle$.

Let $G=\bigcap_{i<\omega} G_{i}$. Clearly $G \subseteq H$ is comeager in $\mathcal{X}^{T}$. To see the additional part, take $K \in \mathcal{K}$. Since $G=\bigcap\left\{G_{i}: i<\omega \& K_{i}=K\right\}$, for every $x \in G$ the set

$$
G_{x \uparrow K}=\bigcap\left\{\left(G_{i}\right)_{x \uparrow K_{i}}: i<\omega \& K_{i}=K\right\}
$$

is comeager in $\mathcal{X}^{T \backslash K}$.

The next lemma is an immediate consequence of Lemma 4.5 applied to $\mathcal{X}=$ $\mathbb{R}, T=\omega$, and $\mathcal{K}=\{\omega \backslash\{n\}: n<\omega\}$.

Lemma 4.6 For every comeager set $G \subseteq \mathbb{R}^{\omega}$ there exists a comeager set $H \subseteq G$ such that for every $x \in H$ and $n<\omega$ the set $H \cap\left(x+\mathbb{R} \cdot \vec{e}_{n}\right)$ is comeager in $x+\mathbb{R} \cdot \vec{e}_{n}$.

The following lemma will facilitate the inductive step in the next theorem. 
Lemma 4.7 Let $G$ be a comeager subset of $\mathbb{R}^{\omega}$ such that

$(\bullet) G \cap\left(x+\mathbb{R} \cdot \vec{e}_{k}\right)$ is comeager in $x+\mathbb{R} \cdot \vec{e}_{k}$ for every $x \in G$ and $k<\omega$.

Let $f$ be a continuous separately nowhere constant function from $G$ into a Polish space $Y$. If $S \in[G]^{<\omega}$ is such that $f$ is one-to-one on $S$, then for every $k<\omega$ and $\varepsilon>0$ there exists a $\delta \in(0, \varepsilon)$ such that $\left(S+\delta \cdot \vec{e}_{k}\right) \subseteq G$ and $f$ is one-to-one on $S \cup\left(S+\delta \cdot \vec{e}_{k}\right)$.

Proof. Let $S=\left\{x_{i}: i<n\right\} \subseteq G$ be such that $f \uparrow S$ is one-to-one. Since $f$ is continuous, decreasing $\varepsilon$ if necessary, we can assume that

(*) if $S^{*}=\left\{x_{i}^{*}: i<n\right\}$ is such that $x_{i}^{*} \in G \cap B\left(x_{i}, \varepsilon\right)$ for every $i<n$, then $f$ is also one-to-one on $S^{*}$.

For each $x \in S$ consider the sets $M_{x}=\left\{\delta \in \mathbb{R}: x+\delta \cdot \vec{e}_{k} \in G\right\}$, which by $(\bullet)$ are comeager, and $N_{x}=\left\{\delta \in M_{x}: f\left(x+\delta \cdot \vec{e}_{k}\right) \in f[S]\right\}$. Since $f \uparrow G \cap\left(x+\mathbb{R} \cdot \vec{e}_{k}\right)$ is nowhere constant, the set $N_{x}$ is meager in $\mathbb{R}$. So, $B=\bigcap_{x \in S} M_{x} \backslash \bigcup_{x \in S} N_{x}$ is comeager in $\mathbb{R}$.

Pick a $\delta \in(0, \varepsilon) \cap B$. Then $S+\delta \cdot \vec{e}_{k} \subseteq G$ as $\delta \in \bigcap_{x \in S} M_{x}$. To see that $f$ is one-to-one on $S \cup\left(S+\delta \cdot \vec{e}_{k}\right)$ take $x \neq y$ in this set. We need to show that $f(x) \neq f(y)$. This follows from the assumption when $x, y \in S$, from (*) when $x, y \in S+\delta \cdot \vec{e}_{k}$, and from $\delta \notin \bigcup_{x \in S} N_{x}$ otherwise.

Theorem 4.8 Let $G$ be a comeager subset of $\mathbb{R}^{\omega}$ and let $f$ be a continuous separately nowhere constant function from $G$ into a Polish space $Y$. Then there is a perfect cube $P$ in $\mathbb{R}^{\omega}$ such that $P \subseteq G$ and $f$ is one-to-one on $P$.

Proof. Let $\left\{m_{k}: k<\omega\right\}$ be an enumeration of $\omega$ where every natural number appears infinitely often. By Lemma 4.6, shrinking $G$ if necessary, we can assume that $G$ satisfies the condition $(\bullet)$ from Lemma 4.7. Since $G$ is a dense $G_{\delta}$ subset of $\mathbb{R}^{\omega}$ we have $G=\bigcap_{n<\omega} G_{n}$, where each $G_{n}$ is open and dense subset of $\mathbb{R}^{\omega}$.

We construct by induction on $k<\omega$ the sequences $\left\langle S_{k} \in[G]^{2^{k}}: k<\omega\right\rangle$, $\left\langle\varepsilon_{k}: k<\omega\right\rangle$, and $\left\langle\delta_{k}: k<\omega\right\rangle$ such that for every $k<\omega$ :

(1) $0<\delta_{k}<\varepsilon_{k} \leq 2^{-k}$

(2) $S_{k+1}=S_{k} \cup\left(\delta_{k} \cdot \vec{e}_{m_{k}}+S_{k}\right) \subseteq \bigcup\left\{B\left(x, \varepsilon_{k}\right): x \in S_{k}\right\}$, 
(3) $\bar{B}\left(x, \varepsilon_{k}\right) \subseteq G_{k}$ for every $x \in S_{k}$,

(4) $f\left[\bar{B}\left(x, \varepsilon_{k}\right)\right] \cap f\left[\bar{B}\left(x^{*}, \varepsilon_{k}\right)\right]=\emptyset$ for every distinct $x, x^{*} \in S_{k}$.

We start the construction with an arbitrary $S_{0}=\{s\} \subseteq G$, and $\varepsilon_{0} \leq 1$ ensuring (3). If for some $k<\omega$ the set $S_{k}$ and $\varepsilon_{k}$ are already constructed we choose $\delta_{k}$ using Lemma 4.7 with $k=m_{k}$ and $\varepsilon \leq \varepsilon_{k}$ small enough that it insures (2) and $\left|S_{k+1}\right|=2^{k+1}$. Then $f$ is one-to-one on $S_{k+1} \subseteq G$ and, using continuity of $f$, we can choose $\varepsilon_{k+1}$ satisfying (1), (3), and (4). This finishes the construction.

If for $n, k<\omega$ we put $A_{k, n}=\left\{x(n): x \in S_{k}\right\}$, then it is easy to see that:

(a) $S_{k}=\prod_{n<\omega} A_{k, n}$,

(b) $A_{k+1, n}=A_{k, n}$ for every $n \neq m_{k+1}$,

(c) $A_{k+1, m_{k+1}}=A_{k, m_{k+1}} \cup\left(\delta_{k}+A_{k, m_{k+1}}\right)$.

We define $P_{n}=\overline{\bigcup_{k<\omega} A_{k, n}}$ and put $P=\prod_{n<\omega} P_{n}$. We will show that each $P_{n}$ is a perfect subset of $\mathbb{R}, P \subseteq G$, and $f$ is one-to-one on $P$. Notice that this will finish the proof, because as a final adjustment (necessary, when $P_{n}$ has a non-empty interior in $\mathbb{R}$ ) we can shrink each $P_{n}$ to a subset from $\operatorname{Perf}(\mathbb{R})$.

Clearly each $P_{n}$ is closed and, by (1) and (2), it has no isolated points. We need to show that

$$
\overline{\bigcup_{k<\omega} S_{k}}=\prod_{n<\omega} P_{n}
$$

The inclusion $\overline{\bigcup_{k<\omega} S_{k}} \subseteq \prod_{n<\omega} P_{n}$ follows from (a). In order to prove the other inclusion pick an $x \in \prod_{n<\omega} P_{n}$. Then for every $n<\omega$ there exists a sequence $\left\{a_{i}^{n}: i<\omega\right\} \subseteq \bigcup_{k<\omega} A_{k, n}$ with distinct terms such that $\lim _{i \rightarrow \infty} a_{i}^{n}=$ $x(n)$. For every $m<\omega$ let $x_{m} \in \mathbb{R}^{\omega}$ be defined as $x_{m}(i)=a_{i}^{m}$ if $i \leq m$ and $x_{m}(i)=s(i)$ if $i>m$. Then $\left\{x_{m}: m<\omega\right\} \subseteq \bigcup_{k<\omega} S_{k}$ and $\lim _{m \rightarrow \infty} x_{m}=x$. This proves that $\prod_{n<\omega} P_{n} \subseteq \overline{\bigcup_{k<\omega} S_{k}}$.

Next, notice that if for $k<\omega$ we put $T_{k}=\bigcup\left\{\bar{B}\left(x, \varepsilon_{k}\right): x \in S_{k}\right\}$ then condition (2) gives us $\overline{\bigcup_{k<\omega} S_{k}} \subseteq \bigcap_{k<\omega} T_{k}$ while the other inclusion is obvious. In particular we have

$$
\prod_{n<\omega} P_{n}=\overline{\bigcup_{k<\omega} S_{k}}=\bigcap_{k<\omega} T_{k}
$$


In order to prove that $f$ is one-to-one on $\prod_{n<\omega} P_{n}$ pick distinct $x$ and $y$ from $\prod_{n<\omega} P_{n}$. Then there are sequences $\left\{x_{m}\right\}$ and $\left\{y_{m}\right\}$ such that for every $m<\omega$ we have $x_{m}, y_{m} \in S_{m}, x \in \bar{B}\left(x_{m}, \varepsilon_{m+1}\right)$, and $y \in \bar{B}\left(y_{m}, \varepsilon_{m+1}\right)$. Since $x \neq y$, there is an $m<\omega$ such that $x_{m} \neq y_{m}$. So, by (5), we have $f\left[\bar{B}\left(x_{m}, \varepsilon_{m+1}\right)\right] \cap f\left[\bar{B}\left(y_{m}, \varepsilon_{m+1}\right)\right]=\emptyset$. Hence $f(x) \neq f(y)$. This shows that $f$ is one-to-one on $\prod_{n<\omega} P_{n}$.

Finally, note that by (3) we have $\prod_{n<\omega} P_{n}=\bigcap_{k<\omega} T_{k} \subseteq G$.

Corollary 4.9 Let $\left\{X_{n}: n<\omega\right\}$ be a family of Polish spaces, $G$ be a dense $G_{\delta}$ subset of $\prod_{n<\omega} \mathcal{X}_{n}$, and let $f$ be a continuous separately nowhere constant function from $G$ into a Polish space $Y$. Then there exist perfect sets $P_{n} \in$ $\operatorname{Perf}\left(\mathcal{X}_{n}\right), n<\omega$, such that $f$ is one-to-one on $\prod_{n<\omega} P_{n}$.

Proof. For every $n<\omega$ let $G_{n}$ be a dense $G_{\delta}$ subset of $\mathcal{X}_{n}$ homeomorphic to the Baire space $\omega^{\omega}{ }^{1}$ Since $\omega^{\omega}$ is homeomorphic to $\mathbb{R} \backslash \mathbb{Q}$, there is a homeomorphism $h_{n}: G_{n} \rightarrow \mathbb{R} \backslash \mathbb{Q}$. Then, $h: \prod_{n<\omega} G_{n} \rightarrow(\mathbb{R} \backslash \mathbb{Q})^{\omega}$ defined by $h=\left\langle h_{n}: n\langle\omega\rangle\right.$ is a cube-preserving homeomorphism. We can apply Theorem 4.8 to the function $f \circ h^{-1}$ on a dense $G_{\delta}$ subset $h\left[G \cap \prod_{n<\omega} G_{n}\right]$ of $\mathbb{R}^{\omega}$ to obtain a perfect cube $\prod_{n<\omega} Q_{n}$ on which $f \circ h^{-1}$ is one-to-one. Then, $h^{-1}\left[\prod_{n<\omega} Q_{n}\right]$ is a perfect cube in $\prod_{n<\omega} \mathcal{X}_{n}$ on which $f$ is one-to-one.

Proof of Theorem 4.1. We can assume that the index set $I$ is a cardinal number $\kappa$. Let $\mathcal{X}=\prod_{i \in \kappa} \mathcal{X}_{i}$.

The case $\kappa=\omega$ is true by Corollary 4.9.

If $\kappa=n<\omega$ and $f: G \rightarrow \mathcal{Y}$ is continuous and separately nowhere constant consider $F: G \times \mathfrak{C}^{\omega \backslash n} \rightarrow \mathcal{Y} \times \mathfrak{C}^{\omega} \backslash n$ defined by $F(x)=(f(x \uparrow n), x \uparrow$ $\omega \backslash n)$. Then, $F$ is continuous and separately nowhere constant function defined on a dense $G_{\delta}$ subset of $\prod_{i \in \omega} \mathcal{X}_{i}$ where $\mathcal{X}_{i}=\mathfrak{C}$ for every $i \in \omega \backslash n$. Thus, by case $\kappa=\omega$, there are $\left\{P_{i} \in \operatorname{Perf}\left(\mathcal{X}_{i}\right): i<\omega\right\}$ such that $F$ is one-to-one on $\prod_{i<\omega} P_{i} \subseteq G \times \mathfrak{C}^{\omega} \backslash n$. This implies that $f$ is one-to-one on $\prod_{i<n} P_{i} \subseteq G$.

If $\kappa>\omega$ then the result is trivial because in this case $f$ cannot be simultaneously continuous and separately nowhere constant on a dense $G_{\delta}$

\footnotetext{
${ }^{1}$ Every Polish space $\mathcal{X}$ (without isolated points) has a dense subspace $G$ homeomorphic to $\omega^{\omega}$ constructed as follows. Let $\left\{B_{n}: n<\omega\right\}$ be a basis for $\mathcal{X}$. Then $\mathcal{Y}=\mathcal{X} \backslash \bigcup_{n<\omega} \operatorname{bd}\left(B_{n}\right)$ is a zero-dimensional dense $G_{\delta}$ subspace of $\mathcal{X}$. Take a countable dense subset $D$ of $\mathcal{Y}$ and put $G=\mathcal{Y} \backslash D$. Then $G$ is a dense $G_{\delta}$ subspace of $\mathcal{X}$. Also, $G$ is Polish, zero-dimensional, and every compact subset of $G$ has an empty interior. So, by Alexandrov-Urysohn theorem [26, thm. 7.7], it is homeomorphic to $\omega^{\omega}$.
} 
subset $G$ of $\mathcal{X}$. To see this first notice that $G$ contains a subset of the form $H \times \prod_{i \in \kappa \backslash A} \mathcal{X}_{i}$, where $A$ is a countable subset of $\kappa$ and $H$ is a dense $G_{\delta}$ subset of $\prod_{i \in A} \mathcal{X}_{i}$. This is the case, since every dense open subset $U$ of $\mathcal{X}$ contains a dense open subset in similar form: a union of a maximal pairwise disjoint family of basic open subsets of $U$. So, we can assume that $G$ is in this form. Pick an $x_{0} \in G$. By the continuity of $f$ at $x_{0}$, for every $n<\omega$ there exists an open subset $U_{n}$ in $\mathcal{X}$ containing $x_{0}$ such that the diameter of $f\left[G \cap U_{n}\right]$ is less than $2^{-n}$. By the definition of the product topology each $U_{n}$ contains a set of the form $\prod_{i \in F_{n}}\left\{x_{0}(i)\right\} \times \prod_{i \in \kappa \backslash F_{n}} \mathcal{X}_{i}$, where each $F_{n} \subseteq \kappa$ is finite. Put $F=A \cup \bigcup_{n<\omega} F_{n}$ and notice that $Z=\prod_{i \in F}\left\{x_{0}(i)\right\} \times \prod_{i \in \kappa \backslash F} \mathcal{X}_{i} \subseteq G \cap \bigcap_{n<\omega} U_{n}$. So, $f[Z]$ has the diameter equal to 0 , that is, $f$ is constant on $Z$. But this contradicts the fact that $f$ is separately nowhere constant on $G$, since for $\xi \in \kappa \backslash F$ set $Z$ contains the section $\left\{x \in \mathcal{X}: x \uparrow \kappa \backslash\{\xi\}=x_{0}\lceil\kappa \backslash\{\xi\}\}\right.$.

\subsection{Cube and prism densities}

Lemma 4.10 For $0<n<\omega$ and any continuous $f: \mathfrak{C}^{n} \rightarrow Y$ there exist a basic clopen subset $U=\prod_{i<n} U_{i}$ of $\mathfrak{C}^{n}$, an $A \subseteq n$, and, if $A \neq n$, a dense $G_{\delta}$ subset $G$ of $W=\prod_{i \in n \backslash A} U_{i}$ such that

- $f \uparrow U$ does not depend on the variables $x_{j}$ for $j \in A$;

- if $A \neq n$ then $f \uparrow U$, considered as a function of the variables $x_{i}$ with $i \in n \backslash A$, is separately nowhere constant on $G$.

Proof. We proceed by induction on $n$. If $n=1$ the lemma is true by the definition of nowhere constant function. Suppose the lemma is true for $n$ and let $f: \mathfrak{C}^{n+1} \rightarrow Y$ be continuous. Denote by $\mathcal{B}_{0}$ a countable basis for the topology on $\mathfrak{C}$ consisting of non-empty clopen sets. For each $i \leq n$ and $V \in \mathcal{B}_{0}$ consider the closed set $S_{i}(V)=\left\{\vec{x} \in \mathfrak{C}^{n+1 \backslash\{i\}}: f\lceil\{\vec{x}\} \times V\right.$ is constant $\}$.

First assume that for every $i \leq n$ and $V \in \mathcal{B}_{0}$ the set $S_{i}(V)$ has empty interior. Then each set $H_{i}=\mathfrak{C}^{\{i\}} \times\left(\mathfrak{C}^{n+1 \backslash\{i\}} \backslash \bigcup\left\{S_{i}(V): B \in \mathcal{B}_{0}\right\}\right)$ is comeager in $\mathfrak{C}^{n+1}$. So, we can apply Lemma 4.5 to $\mathcal{X}=\mathfrak{C}, T=n+1, \mathcal{K}=\{n+1 \backslash$ $\{i\}: i \leq n\}$, and $H=\bigcap_{i \leq n} H_{i}$ to find a comeager set $G \subseteq H$ such that for every $x \in G$ and $i \leq n$ the set $G_{x\lceil n+1 \backslash\{i\}}=\left\{y \in \mathfrak{C}^{\{i\}}:(x\lceil n+1 \backslash\{i\}) \cup y \in G\}\right.$ is comeager in $\mathfrak{C}^{\{i\}}$. Note also that this last property implies that $f\lceil G$ is separately nowhere-constant, since for every $x \in G$ its restriction $x\lceil n+1 \backslash\{i\}$ 
does not belong to $\bigcup\left\{S_{i}(V): B \in \mathcal{B}_{0}\right\}$. Thus, in this case the lemma is satisfied with $U=\mathfrak{C}^{n+1}, A=\emptyset$, and the above chosen $G$

So, assume that there exist $i \leq n$ and $V_{i} \in \mathcal{B}_{0}$ such that the set $S_{i}\left(V_{i}\right)$ has non-empty interior. Let $V^{*} \subseteq S_{i}\left(V_{i}\right)$ be a non-empty basic clopen subset of $\mathfrak{C}^{n+1 \backslash\{i\}}$. Then $V^{*}=\prod_{j \neq i} V_{j}$, where $V_{j} \subseteq \mathfrak{C}$ is a basic clopen set for every $j \neq i$. If $V=\prod_{j \leq n} V_{j}$ then $V$ is homeomorphic to $\mathfrak{C}^{n+1}, f \uparrow V$ does not depend on the variable $x_{i}$, and we can consider $f \uparrow V$ as a function $g$ from $V^{*}$ to $Y$. By applying our inductive hypothesis to $g$ we can find a basic clopen subset $U^{*}=\prod_{j \neq i} U_{j}$ of $V^{*}$, a set $A^{*} \subseteq n+1 \backslash\{i\}$, and, if $A^{*} \neq n+1 \backslash\{i\}$, a dense $G_{\delta}$ subset $G$ of $W=\prod_{n+1 \backslash A} U_{j}$, where $A=A^{*} \cup\{i\}$, satisfying the lemma for $g$. But then $U=\prod_{j \leq n} U_{j}$, where $U_{i}=V_{i}$, and the sets $A$ and $G$ are as desired.

Here is the main example of this chapter.

Example 4.11 For every $0<\alpha<\omega_{1}$ there is a family $\mathcal{G}_{\alpha} \subseteq \operatorname{Perf}\left(\mathfrak{C}^{\alpha}\right)$ such that

(a) $\mathcal{G}_{\alpha}$ does not contain any iterated perfect set, that is, $\mathcal{G}_{\alpha} \cap \mathbb{P}_{\alpha}=\emptyset$;

(b) $\mathcal{G}_{\alpha}$ is $\gamma$-prism dense for every $0<\gamma<\alpha$;

(c) $\mathcal{G}_{\alpha}$ is $n$-cube dense for every $0<n<\min \{\alpha, \omega\}$;

(d) if $\mathcal{G}^{*} \in\left[\mathcal{G}_{\alpha}\right]^{<\mathfrak{c}}$ then $\left|\mathfrak{C}^{\alpha} \backslash \bigcup \mathcal{G}^{*}\right|=\mathfrak{c}$.

Proof. For $\xi<\alpha$ let $\mathcal{K}_{\xi}=\left\{P \in \operatorname{Perf}\left(\mathfrak{C}^{\alpha}\right): \pi_{\xi} \uparrow \pi_{\xi+1}[P]\right.$ is one-to-one $\}$, where in case of $\xi=0$ we put $\mathcal{K}_{0}=\left\{P: \pi_{1}[P]\right.$ is a singleton $\}$. It is worth to note that $\left\{P \in \operatorname{Perf}\left(\mathfrak{C}^{\alpha}\right): \pi_{\xi} \uparrow P\right.$ is one-to-one $\} \subseteq \mathcal{K}_{\xi}$. Then, we define $\mathcal{G}_{\alpha}=\bigcup_{\xi<\alpha} \mathcal{K}_{\xi}$.

To see (a) take $P \in \mathbb{P}_{\alpha}$ and $\xi<\alpha$. We need to show that $P \notin \mathcal{K}_{\xi}$. But by $(\mathrm{C})$ we have $\pi_{\xi+1}[P] \in \mathbb{P}_{\xi+1}$ and then (B) shows that $P \notin \mathcal{K}_{\xi}$.

We prove (b) by induction on $\alpha$. Clearly it holds for $\alpha=1$. So, assume that for some $1<\alpha<\omega_{1}$ condition (b) holds for every non-zero $\alpha^{\prime}<\alpha$. To see that (b) holds for $\alpha$ fix $0<\gamma<\alpha$ and a continuous injection $f: \mathfrak{C}^{\gamma} \rightarrow \mathfrak{C}^{\alpha}$. We need to find a $Q \in \mathbb{P}_{\gamma}$ for which $f[Q] \in \mathcal{G}_{\alpha}$.

Let $g=\pi_{\gamma} \circ f$. By $\left[10\right.$, Lemma 3.2.2] there exist $P \in \mathbb{P}_{\gamma}$ and $0<\beta \leq \gamma$ such that $h=g \circ \pi_{\beta}^{-1}$ is a function on $\pi_{\beta}[P]$ (i.e., $g \uparrow P$ does not depend on coordinates $\delta \geq \beta$ ) and this function is either one-to-one or constant. If $h$ is 
constant then $\pi_{1}[f[P]]$ is a singleton and $f[P] \in \mathcal{K}_{0} \subseteq \mathcal{G}_{\alpha}$. So, assume that $h$ is one-to-one.

If $\beta=\gamma$, then $g=\pi_{\gamma} \circ f$ is one-to-one on $P$ and so $\pi_{\gamma}$ is one-to-one on $f[P]$. Then $f[P] \in \mathcal{K}_{\gamma} \subseteq \mathcal{G}_{\alpha}$. So, assume that $\beta<\gamma$. Then $h$ is an injection from $\pi_{\beta}[P] \in \mathbb{P}_{\beta}$ into $\mathfrak{C}^{\gamma}$. Let $\varphi \in \Phi_{\beta}$ witness $\pi_{\beta}[P] \in \mathbb{P}_{\beta}$. Then $\varphi$ maps $\mathfrak{C}^{\beta}$ onto $\pi_{\beta}[P]$. Since $h \circ \varphi: \mathfrak{C}^{\beta} \rightarrow \mathfrak{C}^{\gamma}$ is a continuous injection, by the inductive hypothesis used for $\alpha^{\prime}=\gamma$ there exists an $E \in \mathbb{P}_{\beta}$ such that $Z=h \circ \varphi[E] \in \mathcal{G}_{\gamma}$, that is, there exists a $\xi<\gamma$ for which $\pi_{\xi}\left\lceil\pi_{\xi+1}[Z]\right.$ is one-to-one.

Next, notice that $R=\varphi[E] \in \mathbb{P}_{\beta}$, since $\Phi_{\beta}$ is closed under the composition, and $R \subseteq \pi_{\beta}[P]$. So, by $(\mathrm{D}), Q=\left\{x \in P: \pi_{\beta}(x) \in R\right\} \in \mathbb{P}_{\alpha}$. Moreover,

$$
Z=h \circ \varphi[E]=h[R]=\left(g \circ \pi_{\beta}^{-1}\right)\left[\pi_{\beta}[Q]\right]=g[P]=\pi_{\gamma}[f[Q]]
$$

and so $\pi_{\xi+1}[Z]=\pi_{\xi+1}\left[\pi_{\gamma}[f[Q]]\right]=\pi_{\xi+1}[f[Q]]$. Thus, $\pi_{\xi \uparrow} \uparrow \pi_{\xi+1}[f[Q]]$ is oneto-one and so $f[Q] \in \mathcal{K}_{\xi} \subseteq \mathcal{G}_{\alpha}$.

To show (c) we will prove by induction on $0<n<\omega$ the statement

for every $0<\alpha<\omega_{1}$ if $n<\alpha$ then $\mathcal{G}_{\alpha}$ is $n$-cube dense.

So, take $0<n<\omega$ and assume that the statement holds for all non-zero $k<$ $n$. Take an $\alpha>n$. To prove that $\mathcal{G}_{\alpha}$ is $n$-cube dense fix a continuous injection $f: \mathfrak{C}^{n} \rightarrow \mathfrak{C}^{\alpha}$. Then $\pi_{n} \circ f: \mathfrak{C}^{n} \rightarrow \mathfrak{C}^{n}$ is continuous. Apply Lemma 4.10 to $\pi_{n} \circ f$ to find $U=\prod_{i<n} U_{i} \subseteq \mathfrak{C}^{n}, A \subseteq n$, and $G$ satisfying the lemma.

If $A=n$, then $\pi_{n}[f[U]]=\left(\pi_{n} \circ f\right)[U]$ is a singleton and $f[U] \in \mathcal{K}_{0} \subseteq \mathcal{G}_{\alpha}$.

If $A=\emptyset$, then $\pi_{n} \circ f \uparrow G$ is continuous separately nowhere constant. So, by Theorem 4.1, there exist perfect sets $\left\{P_{i} \subseteq U_{i}: i<n\right\}$ such that $\left(\pi_{n} \circ f\right) \uparrow \prod_{i<n} P_{i}$ is one-to-one. Then, $\pi_{n} \uparrow f\left[\prod_{i<n} P_{i}\right]$ is one-to-one and so $f\left[\prod_{i<n} P_{i}\right] \in \mathcal{K}_{n} \subseteq \mathcal{G}_{\alpha}$.

So, assume that $\emptyset \neq A \neq n$ and let $k=|n \backslash A|$. Then $0<k<n$. Since $\left(\pi_{n} \circ f\right) \uparrow U$ does not depend on the variables $x_{j}$ for $j \in A$, it can be considered as a function $g$ on $W=\prod_{i \in n \backslash A} U_{i}$. Moreover, $g\lceil G$ is separately nowhere constant. Thus, by Theorem 4.1, we can find a perfect cube $P=\prod_{i \in n \backslash A} P_{i} \subseteq$ $G \subseteq \prod_{i \in n \backslash A} U_{i}$ on which $g$ is one-to-one. Thus, $g$ is a continuous injection from $P$, which can be identified with $\mathfrak{C}^{k}$, into $\mathfrak{C}^{n}$. Since, by the inductive assumption, $\mathcal{G}_{n}$ is $k$-cube dense, there exists a perfect cube $C=\prod_{i \in n \backslash A} C_{i} \subseteq$ $\prod_{i \in n \backslash A} P_{i}$ such that $g[C] \in \mathcal{G}_{n}$. Let $C_{i}=U_{i}$ for $i \in A$. Then $Q=\prod_{i<n} C_{i} \subseteq$ $\prod_{i<n} U_{i}$ is a perfect cube and $\pi_{n}[f[Q]]=\left(\pi_{n} \circ f\right)[Q]=g[C] \in \mathcal{G}_{n}$. So, there 
exists a $\xi<n$ such that $\pi_{\xi}$ is one-to-one on $\pi_{\xi+1}\left[\pi_{n}[f[Q]]\right]=\pi_{\xi+1}[f[Q]]$, So, $f[Q] \in \mathcal{K}_{\xi} \subseteq \mathcal{G}_{\alpha}$.

Now, to argue for (d) fix a $\mathcal{G}^{*} \in\left[\mathcal{G}_{\alpha}\right]^{<\mathfrak{c}}$. We need to show that $\left|\mathfrak{C}^{\alpha} \backslash \bigcup \mathcal{G}^{*}\right|=$ c. For $\xi<\alpha$ let $\mathcal{G}_{\xi}^{*}=\mathcal{G}^{*} \cap \mathcal{K}_{\xi}$. By induction on $\xi<\alpha$ choose

$$
x(\xi) \in \mathfrak{C} \backslash\left\{z(\xi): z \in \mathcal{G}_{\xi}^{*} \& z(\eta)=x(\eta) \text { for every } \eta<\xi\right\} .
$$

Note that at each step we have less than continuum many restricted points since for every $z \in \mathcal{K}_{\xi}$ the set $\{z(\xi): z(\eta)=x(\eta)$ for every $\eta<\xi\}$ may have at most one element. It is easy to see that $x=\langle x(\xi): \xi<\alpha\rangle \in \mathfrak{C}^{\alpha} \backslash \bigcup_{\xi<\alpha} \mathcal{G}_{\xi}^{*}=$ $\mathfrak{C}^{\alpha} \backslash \mathcal{G}^{*}$. To finish the proof it is enough to notice that the value of $x(0)$ can be chosen in continuum many ways, so indeed $\left|\mathfrak{C}^{\alpha} \backslash \bigcup \mathcal{G}^{*}\right|=\mathfrak{c}$.

To transport the above example into an arbitrary Polish space we will use the following simple fact.

Remark 4.12 Let $h$ be a homeomorphic embedding of a Polish space $Y$ into a Polish space $\mathcal{X}$, let $\mathcal{F} \subseteq \operatorname{Perf}(Y)$, and put $\mathcal{F}^{*}=\{h[F]: F \in \mathcal{F}\} \cup$ $\operatorname{Perf}\left(\mathcal{X} \backslash h\left[Y[)\right.\right.$. Then for every $1 \leq \alpha \leq \omega_{1}$ the following conditions are equivalent.

(a) $\mathcal{F}$ is $\alpha$-cube $(\alpha$-prism) dense in $Y$.

(b) $\mathcal{F}^{*}$ is $\alpha$-cube $(\alpha$-prism) dense in $\mathcal{X}$.

Proof. "(a) $\Longrightarrow(\mathrm{b})$ " Let $f: \mathfrak{C}^{\alpha} \rightarrow \mathcal{X}$ be injective and continuous. Since $h[Y]$ is a $G_{\delta^{-}}$set in $\mathcal{X}$ we can apply [12, Claim 3.2] (see also [10, Claim 1.21.5]) to find a perfect cube $C \subseteq \mathfrak{C}^{\alpha}$ such that either $f[C] \subseteq h[Y]$ or $f[C] \cap h[Y]=\emptyset$.

If $f[C] \cap h[Y]=\emptyset$ then $f[C] \in \mathcal{F}^{*}$ and we are done. If $f[C] \subseteq h[Y]$ then $h^{-1} \circ f: C \rightarrow Y$ is a continuous injection. Identifying $C$ with $\mathfrak{C}^{\alpha}$ and using to $h^{-1} \circ f$ the $\alpha$-cube ( $\alpha$-prism) density of $\mathcal{F}$ in $Y$ we can find a $C^{\prime} \subseteq C$ such that $C^{\prime}$ is a perfect cube (belongs to $\left.\mathbb{P}_{\alpha}\right)$ and $F=\left(h^{-1} \circ f\right)\left[C^{\prime}\right] \in \mathcal{F}$. So $f\left[C^{\prime}\right]=h[F] \in \mathcal{F}^{*}$. The family $\mathcal{F}^{*}$ is as desired.

The other implication is easy.

Corollary 4.13 For every $1<\alpha<\omega_{1}$ and every Polish space $\mathcal{X}$ there exists a family $\mathcal{F}_{\alpha} \subseteq \operatorname{Perf}(\mathcal{X})$ such that: $\mathcal{F}_{\alpha}$ is not $\alpha$-prism dense; $\mathcal{F}_{\alpha}$ is $\beta$-prism dense for every $0<\beta<\alpha ; \mathcal{F}_{\alpha}$ is $n$-cube dense for every $0<n<\min \{\alpha, \omega\}$; $\left|\mathcal{X} \backslash \bigcup \mathcal{F}_{\alpha}\right|=\mathfrak{c}$. 
Proof. First note that it is enough to find such an $\mathcal{F}_{\alpha}$ for $\mathcal{X}=\mathfrak{C}^{\alpha}$. Indeed, if $\mathcal{F}$ is such a family and $h, \mathcal{X}$ is an arbitrary Polish space, and $h$ is an embedding from $\mathfrak{C}^{\alpha}$ into $\mathcal{X}$, then the family $\mathcal{F}^{*}$ from Remark 4.12 is as desired.

Thus, it is enough to notice that the family $\mathcal{G}_{\alpha}$ from Example 4.11 is not $\alpha$-prism dense. But this is the case since for the identity function $f$ on $\mathfrak{C}^{\alpha}$ there is no $P \in \mathbb{P}$ for which $f[P]=P \in \mathcal{G}_{\alpha}$.

Proof of Theorem 4.4. Use Corollary 4.13 with $\alpha \geq \omega$.

Proof of Theorem 4.3. The vertical implications, that $\alpha$-cube density implies $\alpha$-prism density, follows from the fact $(\mathrm{A})$, that every perfect cube in $\mathfrak{C}^{\alpha}$ is also in $\mathbb{P}_{\alpha}$. For $0<\beta<\alpha<\omega_{1}$ the implications " $\mathrm{C}_{\alpha} \Longrightarrow \mathrm{C}_{\beta}$ " and " $\mathrm{P}_{\alpha} \Longrightarrow \mathrm{P}_{\beta}$ " are obvious. $\mathrm{P}_{2}$ implies $\mathrm{C}_{2}$ since 1-prism density is just perfect set density $\left(\mathbb{P}_{1}=\operatorname{Perf}\left(\mathfrak{C}^{1}\right)\right.$, as $\Phi_{1}$ consists just of autohomeomorphisms of $\left.\mathfrak{C}^{1}\right)$ and so it implies 1-cube density.

To see that for $\omega<\alpha<\omega_{1}$ we have " $\mathrm{C}_{\omega+1} \Longrightarrow \mathrm{C}_{\alpha}$ " it is enough to notice that any $\omega$-cube dense family is also $\beta$-cube dense for any $\omega \leq \beta<\omega_{1}$. This is the case since the coordinatewise homeomorphism between $\mathfrak{C}^{\omega}$ and $\mathfrak{C}^{\beta}$ preserves perfect cubes.

The fact that no other horizontal implication can be reversed is justified by the family $\mathcal{F}_{\alpha}$ from Corollary 4.13 for different values of $\alpha$. Indeed, $\mathcal{F}_{\alpha}$ clearly justifies " $\mathrm{P}_{\alpha} \not \mathrm{P}_{\gamma}$ " for any $1<\alpha<\gamma$ since it satisfies $\mathrm{P}_{\alpha}$ but not $\mathrm{P}_{\gamma}$ as it is not $\alpha$-prism dense. If $1<m<n \leq \omega$ then $\mathcal{F}_{m}$ also witness " $\mathrm{C}_{m} \Rightarrow \mathrm{C}_{n}$ " since it satisfies $\mathrm{C}_{m}$ but not $\mathrm{C}_{n}$, since it cannot be $m$-cube dense without being $m$-prism dense.

The fact that none of the vertical implications " $\mathrm{C}_{\alpha} \Longrightarrow \mathrm{P}_{\alpha}$ ", for $2<\alpha<$ $\omega_{1}$, can be reversed is justified by any family which is $\alpha$-prism dense for every $\alpha$ but is not 2 -cube dense. There are many such families. For example, this is the case for the family $\mathcal{F}$ of all linearly independent (over $\mathbb{Q}$ ) subsets of $\mathbb{R}$. It is shown in [11] (see also [10, Cor. 5.1.2]) that this $\mathcal{F}$ is $\alpha$-prism dense for every $0<\alpha<\omega_{1}$. On the other hand it is not 2-cube dense, as shown by the following function $f$. (See [11, Remark 5.2] or [10, Remark 5.1.4].) Let $P_{1}$ and $P_{2}$ be disjoint perfect subsets of $\mathbb{R}$ such that $P_{1} \cup P_{1}$ is linearly independent over $\mathbb{Q}$. Let $f: P_{1} \times P_{2} \rightarrow \mathbb{R}$ be defined by $f\left(x_{1}, x_{2}\right)=x_{1}+x_{2}$. Identifying $P_{1}$ and $P_{2}$ with $\mathfrak{C}$ we think about $f$ as defined on $\mathfrak{C}^{2}$. It is easy to see that if each of the sets $Q_{1} \subseteq P_{1}$ and $Q_{2} \subseteq P_{2}$ has at least two elements then $f\left[Q_{1} \times Q_{2}\right]$ is linearly dependent.

Another such example is a family $\mathcal{F}$ of all $P \in \operatorname{Perf}\left(\mathfrak{C}^{2}\right)$ such that the 
projection on one of the coordinates is one-to-one. It follows quite easily from [10, Lemma 3.2.2] that $\mathcal{F}$ is $\alpha$-prism dense for every $\alpha$. (See e.g. [10, prop. 4.1.3].) It is not 2-cube dense since for the identity function $f: \mathfrak{C}^{2} \rightarrow \mathfrak{C}^{2}$ there is no perfect cube $C$ for which $f[P] \in \mathcal{F}$.

\subsection{Final remarks}

It is also worth to notice that we have the following implications.

Proposition 4.14 If $\beta+1 \leq \alpha<\omega_{1}$ then every $\alpha$-prism ( $\alpha$-cube) dense family is also $(\beta+1)$-prism $((\beta+1)$-cube) dense. In particular, if $0<m<$ $n \leq \omega$ then every $n$-cube dense family is also $m$-cube dense.

Proof. Let $g: \mathfrak{C}^{\alpha \backslash \beta} \rightarrow \mathfrak{C}$ be a homeomorphism, and let $h: \mathfrak{C}^{\alpha} \rightarrow \mathfrak{C}^{\beta+1}$ be defined by $h(x)(\xi)=x(\xi)$ for every $\xi<\beta$ and $h(x)(\beta)=g(x \uparrow \alpha \backslash \beta)$. It is easy to see that $h$ is a homeomorphism and that if $P \subseteq \mathfrak{C}^{\alpha}$ is a perfect cube (belongs to $\mathbb{P}_{\alpha}$ ) then $h[P]$ is a perfect cube (belongs to $\mathbb{P}_{\beta+1}$ ).

Now, let $\mathcal{F} \subseteq \operatorname{Perf}(\mathcal{X})$ be $\alpha$-prism $\alpha$-cube) dense in $\mathcal{X}$. To see that $\mathcal{F}$ is $(\beta+1)$-prism $((\beta+1)$-cube $)$ dense take a continuous injection $f: \mathfrak{C}^{\beta+1} \rightarrow \mathcal{X}$. Then $f \circ h: \mathfrak{C}^{\alpha} \rightarrow \mathcal{X}$ is also a continuous injection. Since $\mathcal{F}$ is $\alpha$-prism $(\alpha$ cube) dense, there exists a $P \subseteq \mathfrak{C}^{\alpha}$ such that $P$ belongs to $\mathbb{P}_{\alpha}$ (is a perfect cube) and $f[h[P]]=(f \circ h)[P] \in \mathcal{F}$. But $h[P]$ belongs to $\mathbb{P}_{\beta+1}$ (is a perfect cube), so $\mathcal{F}$ is $(\beta+1)$-prism $((\beta+1)$-cube) dense.

We do not know if, in general, for a limit ordinal $\lambda<\omega_{1}$ the $(\lambda+1)$-prism density implies $\lambda$-prism density.

The next example shows that Lemma 4.10 fails, in a strong way, for functions defined on infinite product.

Example 4.15 There exists a continuous function $f: \mathfrak{C}^{\omega} \rightarrow \mathfrak{C}^{\omega}$ such that for every perfect cube $P$ there is an $n<\omega$ such that $f \uparrow P$ is one-to-one on some section of $n$-th variable, and is constant on some other sections of the same variable.

Proof. For $n<\omega$ let $f_{n}: \mathfrak{C}^{2} \rightarrow \mathfrak{C}$ be defined by $f_{n}(x, y)(i)=y(n) \cdot x(i)$. Clearly $f_{n}$ is continuous. Moreover, if $y(n)=1$, then $f_{n}(\cdot, y)$ is the identity function, so it is one-to-one; if $y(n)=0$ then $f_{n}(\cdot, y)$ is constant equal to 0 . 
For $\left\langle x_{n}: n<\omega\right\rangle \in \mathfrak{C}^{\omega}$ define $f\left(\left\langle x_{n}: n<\omega\right\rangle\right)=\left\langle f_{n}\left(x_{n+1}, x_{0}\right): n<\omega\right\rangle$. Then $f$ is clearly continuous. Consider $f$ restricted to a perfect cube $P=$ $\prod_{n<\omega} P_{n}$. Let $a, b \in P_{0}$ be distinct and let $n<\omega$ be such that $a(n) \neq b(n)$. Assume that $a(n)=0$ and let $z \in \mathfrak{C}^{\omega \backslash\{n+1\}}$. Look at $f \uparrow P$ on a section given by $z$ and note that: if $z(0)=a$, then $f \uparrow P$ is constant on this section; if $z(0)=b$, then $f \uparrow P$ is one-to-one on this section. 


\section{Bibliography}

[1] Bartoszyński T., Judah, H. Set Theory, on the structure of the real line, A.K. Peters, 1995.

[2] Bartoszyński T., Reclaw, I. Not every $\gamma$-set is strongly meager., Set Theory (Boise, ID, 1992-1994), 25-29, Contemp. Math. 192 Amer. Math. Soc., Providence, RI, 1996.

[3] Baumgartner, J., Laver, R. Iterated perfect set forcing, Ann. Math. Logic 17 (1979), 271-288.

[4] Blass, A., Combinatorial Characteristics of the Continuum, Handbook of Set Theory, to appear.

[5] Brodskiı̌, M.L. On some properties of sets of positive measure, Uspehi Matem. Nauk. (N.S) 4, No.3 (31) (1949), 136-138.

[6] Cohen, P.J., The independence of the continuum hypothesis, Proc. Nat. Acad. U.S.A. 50 (1963) 1143-1148; 51 (1964), 105-110.

[7] Ciesielski, K., Millán, A., Pawlikowski, J. Uncountable $\gamma$-sets and CPA ${ }_{\text {cube }}^{\text {game }}$ Fund. Math. 176 (2003), 143-155.

[8] Ciesielski, K., Millán, A. n-cube and $\alpha$-prism densities, J. Appl. Anal., to appear.

[9] Ciesielski, K., Pawlikowski, J. Crowded and selective ultrafilters under the Covering Property Axiom, J. Appl. Anal. 9(1) (2003), 19-55.

[10] Ciesielski, K., Pawlikowski, J. Covering Property Axiom CPA. A combinatorial core of the iterated perfect set model., Cambridge Tracts in Mathematics 164, Cambridge University Press, 2004. 
[11] Ciesielski, K., Pawlikowski, J. Nice Hamel bases under the Covering Property Axiom, Acta Math. Hungar. 105(3) (2004), 197-213.

[12] Ciesielski, K., Pawlikowski, J. Covering Property Axiom $\mathrm{CPA}_{\text {cube }}$ and its consequences, Fund. Math. 176(1) (2003), 63-75.

[13] Čoplakova, E., and Hart, K.P. Crowded rational ultrafilters, Topology Appl. 97 (1999), 79-84.

[14] van Douwen, E.K. Better closed ultrafilters on $\mathbb{Q}$, Topology Appl. 47 (1992), 173-177.

[15] Eggleston, H.G. Two measure properties of Cartesian products sets, Quart. J. Math. Oxford (2) (5) (1954), 108-115.

[16] Franklin, S.P. Spaces in which sequences suffice Fundamenta Mathematicae (57) (1965), 107-115.

[17] Frolík, Z. Homogeneity problems for extremally disconnected spaces, Comm. Math. Univ. Carolinae 8 (1976), 757-763.

[18] Galvin, F., and Miller, A.W. $\gamma$-sets and other singular sets of real numbers, Topology Appl. 17 (1984), 145-155.

[19] Gerlits, J., Nagy, Zs. Some properties of $C(X)$, I, Topology Appl. 14 (1982), 151-161.

[20] Gödel, K. The consistency of the Axiom of Choice and the Generalized Continuum Hypothesis, Proc. Nat. Acad. U.S.A. 24 (1938), 556-557.

[21] Gödel, K. Consistency-proof for the generalized continuum hypothesis, Proc. Nat. Acad. U.S.A. 25 (1939), 220-224.

[22] Gödel, K. The consistency of the axiom of choice and the generalized continuum hypothesis, Ann. Math. Studies 3 (1940).

[23] Hart, K.P. Ultrafilters of character $\omega_{1}$, J. Symbolic Logic 54(1) (1989), $1-15$.

[24] Jech, T. Set Theory, Academic Press, New York, 1978.

[25] Jensen, R.B. The fine structure of the constructible hierarchy, Ann. Math. Logic 4 (1972), 229-308. 
[26] Kechris, A.S. Classical Descriptive Set Theory, Graduate Text in Mathematics 156, Springer Verlag, 1991.

[27] Kunen, K. Weak P-points in $N^{*}$, Colloquia Mathematica Societatis János Bolyai, 23. Topology, Budapest (Hungary), (1978), 741-749.

[28] Kunen, K. Ultrafilters and independent sets, Trans. Amer. Math. Soc. 172 (1972), 299-306.

[29] Kunen, K. Some points in $\beta \mathrm{N}$ Math. Proc. Cambridge Phil. Soc. 80 (1976), 385-398.

[30] Kunen, K. Set Theory, and introduction to independence proofs. Studies in Logic and the Foundations of Mathematics 102, North Holland 1980.

[31] Kuratowski, K. Topology, Vol. I Academic Press, New York, 1966.

[32] Laver, R. On the consistency of Borel's Conjecture, Acta Math. 137 (1976), 151-169.

[33] Martin, D.A., Solovay,R. Internal Cohen Extensions Ann. Math. Logic 2 (1970), 143-178.

[34] McCoy, R.A. k-space function spaces Internat. J. Math. and Math. Sci., 3 (1980), 701-711.

[35] van Mill, J. An Introduction to $\beta \omega$, Handbook of Set-Theoretic Topology, K.Kunen, and J.E.Vaughan (Editors) Elsevier Science Publishers B.V. 1984.

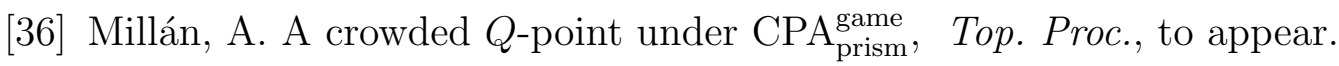

[37] Miller, A.W. There are no $Q$-points in Laver's model for the Borel Conjecture, Proc. Amer. Math. Soc. 78(1) (1980), 103-106.

[38] Miller, A.W. Mapping a set of reals onto the reals, J. Symbolic Logic 48 (1983), 575-584.

[39] Miller, A.W. Special Subsets of the Real Line, in :Handbook of Set-Theoretic Topology (K.Kunen and J.E.Vaughan, Editors), NorthHolland, (1984), 201-233. 
[40] Pospiśíl, B. Remark on Bicompact Spaces Ann. of Math. 38 (1937) 845846.

[41] Reclaw, I. Every Lusin set is undetermined in the point-open game, Fund. Math. 144 (1994), 43-54.

[42] Sacks, G. Forcing with perfect closed sets, "Axiomatic Set Theory" Proc. Symp. Pure Math., 13, II (D. Scott, ed.), 331-355. Amer. Math. Soc., Providence, Rhode Island, 1971.

[43] Solovay, R., Tennembaum, S. Iterated Cohen Extensions and Souslin's Problem, Ann. of Math. 94 (1971), 201-245.

[44] Rudin, W. Homogeneity problems in the theory of Čech compactifications, Duke Math. J. 23 (1956), 409-419.

[45] Wimmers, E. The Shelah P-point independence theorem, Israel J. Math. 43 (1982) 28-48.

[46] Zapletal, J. Descriptive Set Theory and Forcing, Memoirs of the Amer. Math. Soc. 167, 2004.

[47] Zermelo, E. Beweis, dass jede Menge wohlgeordner werdern kann, Math. Ann. 59 (1904) 514-516. 